\title{
Aeroelastic and Aerothermoelastic Analysis of Hypersonic Vehicles: Current Status and Future Trends
}

\author{
Jack J. McNamara* and Peretz P. Friedmann ${ }^{\dagger}$ \\ Department of Aerospace Engineering, The University of Michigan, Ann Arbor, MI, 48109, USA
}

\begin{abstract}
A review of the state-of-the-art in hypersonic aeroelasticity and aerothermoelasticity is provided. Recently, the main focus in this area has been on the development of computational aeroelastic and aerothermoelastic methods capable of studying complete hypersonic vehicles. Thus, in addition to a survey of studies conducted in this area over the past six decades, two important issues are a focus of this review, namely: 1) modeling unsteady hypersonic aerodynamics; and 2) incorporation of the heat transfer between the fluid and the structure into the aeroelastic solution process. Finally, future directions of hypersonic aeroelasticity and aerothermoelasticity are outlined. Since air-breathing hypersonic vehicles exhibit strong coupling between the airframe, propulsion, and control systems, future directions point to the incorporation of advanced computational aerothermoelastic methods into a comprehensive vehicle analysis.
\end{abstract}

\section{Nomenclature}

$a$

$a^{(i)}, b^{(i)}$

$a_{\infty}$

$a_{l}$

$c$

$C_{L}, C_{M}, C_{D}$

$C_{p}$

$\left(C_{p}\right)_{\max }$

$C_{p P T}, C_{p N S}$

$c_{p w}$

$C F L_{\tau}$

$C_{w}$

$h_{h t}$

$K_{h}, K_{\alpha}$

$k_{\omega}$

$M_{\infty}$

$M_{f}$

$M_{N}$

$\mathbf{n}$

$p$

$p_{N}$

$p_{\infty}$
Nondimensional offset between the elastic axis and the midchord, positive for elastic axis locations behind midchord

Coefficients of the perturbation potential

Speed of sound

Local speed of sound

Reference length, Chord length

Coefficients of lift, moment about the elastic axis, and drag

Coefficient of pressure

Coefficient used in modified Newtonian impact theory

Steady piston theory and Navier-Stokes pressure coefficient, respectively

Specific heat of the wall

CFL3D input parameter regulating "pseudo time step size

Chapman-Rubesin coefficient

Heat transfer coefficient

Spring constants in pitch and plunge respectively

Reduced frequency

Free stream Mach number

Flutter Mach number

Mach number at the nose/leading edge of a vehicle

Normal vector

Pressure

Pressure at the nose/leading edge of a vehicle

Free-stream pressure

*Postdoctoral Scholar, Member AIAA, currently Assistant Professor, Aerospace Eng. Dept., Ohio State University, Columbus, $\mathrm{OH}$

${ }^{\dagger}$ François-Xavier Bagnoud Professor, Fellow AIAA. 


\begin{tabular}{|c|c|}
\hline$p_{w}$ & Surface pressure \\
\hline$q_{\infty}$ & Dynamic pressure \\
\hline$q_{v f}$ & Virtual flutter dynamic pressure \\
\hline$\dot{q}_{a e r o}, \dot{q}_{\text {rad }}$, & Heat transfer rate due to aerodynamic heating, radiation, conduction, \\
\hline$\dot{q}_{\text {cond }}, \dot{q}_{\text {strd }}$ & and stored energy respectively \\
\hline$R e$ & Reynolds number \\
\hline$T$ & Temperature \\
\hline$T_{\infty}$ & Freestream temperature \\
\hline$T_{0}$ & Stagnation temperature \\
\hline$T_{W}$ & Wall temperature \\
\hline$T_{A W}$ & Adiabatic wall temperature \\
\hline$T_{\text {Ref }}$ & Reference temperature \\
\hline$t$ & Time \\
\hline$\Delta t$ & Time step \\
\hline $\mathbf{U}_{\infty}$ & Velocity vector \\
\hline$(u, v, w)$ & Local velocity components in $x, y$, and $z$ directions \\
\hline$V_{\infty}$ & Free stream velocity \\
\hline$v_{n}$ & Normal velocity of airfoil surfaces \\
\hline$w_{d}$ & Displacement of the surface of the structure \\
\hline$x, y, z$ & Spatial Coordinates \\
\hline$x_{\alpha}$ & Nondimensional offset between the elastic axis and the cross-sectional center of gravity \\
\hline$Z(x, y, t)$ & Position of structural surface \\
\hline$Z_{\text {str }}(x, y)$ & Function describing surface geometry \\
\hline$Z_{e f f}(x, y)$ & Effective shape on a surface due to a viscous boundary layer \\
\hline$Z_{f p}(x, y)$ & Effective shape on a flat plate due to a viscous boundary layer \\
\hline$\alpha_{s}$ & Angle-of-attack \\
\hline$\beta$ & $=\sqrt{M^{2}-1}$ \\
\hline$\beta_{s}$ & Shock angle of an oblique shock \\
\hline$\Delta_{w}$ & Skin thickness \\
\hline$\delta$ & Maximum of airfoil thickness or surface inclination to the flow \\
\hline$\epsilon$ & Emissivity \\
\hline $\bar{\epsilon}$ & Parameter much less than unity \\
\hline$\gamma$ & Ratio of specific heats \\
\hline$\theta_{l}$ & Local inclination angle to the flow \\
\hline$\theta_{N}$ & Flow deflection angle at the nose/leading edge of a vehicle \\
\hline$\Delta \theta$ & Difference between the local flow deflection angle and the nose deflection angle \\
\hline$\kappa$ & Thermal conductivity \\
\hline$\mu_{w}$ & Fluid dynamic viscosity at the wall \\
\hline$\omega_{i}$ & Frequency of mode $i$ \\
\hline$\Phi_{\text {pot }}$ & Perturbation potential \\
\hline$\phi_{i}$ & Vector of displacements for mode $i$ \\
\hline$\tilde{\Omega}$ & Nonlinear velocity potential \\
\hline$\rho$ & Air density \\
\hline$\rho_{W}$ & Wall density \\
\hline$\sigma$ & Stefan-Boltzmann constant \\
\hline$\zeta$ & Damping ratio \\
\hline
\end{tabular}

\section{Introduction}

In February of 1949 a WAC Coporal rocket was ignited from a US captured V-2 rocket, becoming the first vehicle to fly at hypersonic velocities. ${ }^{1}$ Currently, despite sizeable investments over the years in vehicles aimed at this flight regime, the ability of sustained hypersonic flight is still lacking. The idea of a Single-Stage-To-Orbit (SSTO) Reusable Launch Vehicle (RLV) that could take off and land on conventional runways, called the National Aerospace Plane (NASP), was seriously examined in the mid 1980's. However, the program was canceled due mainly to design requirements that exceeded the state-of-the-art. ${ }^{1,2} \mathrm{~A}$ more 
recent RLV project, the VentureStar program, failed during structural tests, again for lack of the necessary technology.

Despite these unsuccessful programs, the continued need for a low-cost SSTO or Two-Stage-To-Orbit (TSTO) RLV, as well as the desire of the US Air Force for unmanned hypersonic vehicles, has re-invigorated hypersonic flight research. Recent advances in Supersonic Combustion Ramjet (SCRamjet) engines, as evident in the NASA Hyper-X experimental vehicle ${ }^{3}$ and University of Queensland HyShot ${ }^{4}$ efforts, have demonstrated the feasibility of this class of vehicles. In the FALCON (Force Application Launch from CONUS) program, the Defense Advanced Research Projects Agency (DARPA) and the US Air Force intend to utilize SCRamjet technologies to develop, by 2025, an autonomous reusable hypersonic cruise vehicle that can strike targets at distances up to 9,000 nautical miles within 2 hours. $^{5}$

The conditions encountered in hypersonic flows, combined with the need to design hypersonic vehicles, have motivated research in the areas of hypersonic aeroelasticity and aerothermoelasticity. It is evident from Fig. 1 that hypersonic vehicle configurations will consist of long, slender lifting body designs. In general, the body and aerodynamic control surfaces are flexible due to minimum-weight restrictions. Furthermore, as shown in Fig. 2, these vehicles operate ${ }^{6-9}$ over a Mach number range of 0 to 15 ; and must fly within the atmosphere for sustained periods of time to meet the needs of an air-breathing propulsion system. ${ }^{10}$ The combined extreme aerodynamic heating, and loading, acting on the airframe produces complex interactions between the flow, dynamics, structure, control and propulsion systems. ${ }^{11-13}$ These interactions have received only limited attention in the past. Moreover, the inability to test aeroelastically and aerothermoelastically scaled models in wind-tunnels, a common practice in the subsonic and supersonic flow regime, implies that aeroelastic simulations are critical for this flight regime.

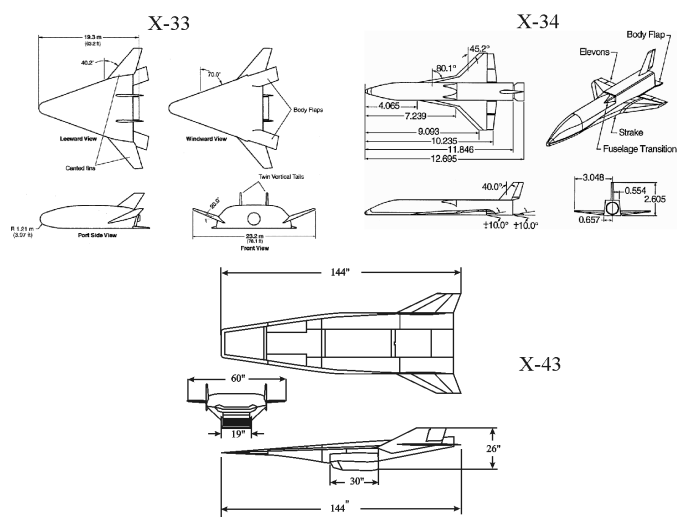

Figure 1. Schematic description of several hypersonic vehicles.

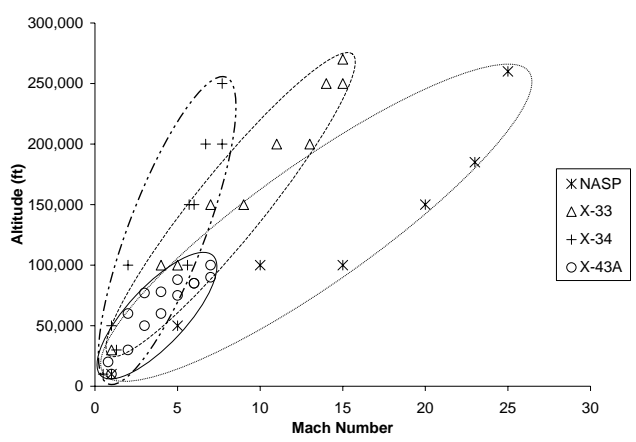

Figure 2. Operating envelopes for several modern hypersonic vehicles.

\section{A. Domain of Aeroelasticity}

Classically, aeroelasticity is defined as the mutual interaction of inertial, elastic, and aerodynamic forces in a particular system, when there is feedback between deformation and flow. ${ }^{14,15}$ However, modern aeroelasticity encompasses a much broader set of problems, as illustrated by the aeroservo-thermo-elastic hexahedron ${ }^{16}$ shown in Fig. 3. In this graphical depiction, classical aeroelasticity is represented by the triangular domain constructed from the Elastic, Inertia, and Aerodynamic vertices. Furthermore, the upper tetrahedron represents the domain of aeroservoelasticity, where the control system is included in the aeroelastic analysis. Similarly, the lower tetrahedron represents the domain of aerothermoelasticity, where thermal effects are included in the aeroelastic analysis.

Hypersonic aeroelasticity and aerothermoelasticity was an active area of research in the late 1950's and during the 1960's as evident from Refs. [17-30]. This research was instrumental in providing the basis for the aerothermoelastic design of the space shuttle. Due to the previously unattainable technology requirements necessary to design and operate hypersonic vehicles, this early research has been followed by periods of inactivity and intermittent spurts of activity. It is clear from recent advances, however, that sustained air-breathing hypersonic flight is on the horizon. Thus, the objective of this paper is to survey the status of research in the area of hypersonic aeroelasticity and aerothermoelasticity in order to provide: 1) a 


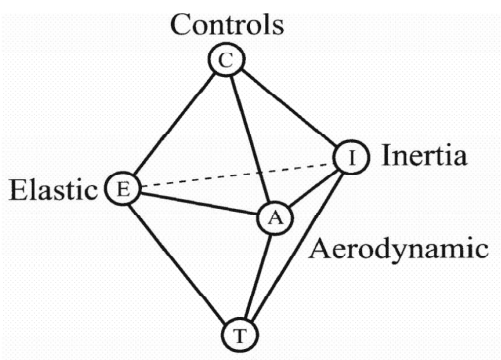

Thermal Effects

Figure 3. Aero-servo-thermo-elastic hexahedron.[16]

comprehensive review of the state-of-the-art, emphasizing some of the authors' research, and 2) insight into important challenges and future directions.

\section{Principal Aspects of the Hypersonic Environment}

In order to provide a comprehensive survey of the current state of hypersonic aeroelastic and aerothermoelastic research, it is helpful to summarize the principal characteristics of hypersonic flow and hypersonic vehicles. The boundary that separates hypersonic flight from supersonic flight is not set at a particular Mach number. Rather, 'hypersonic' is best defined as that regime where certain physical phenomena become progressively more important as the Mach number is increased to higher values. ${ }^{1}$ An important consequence of high speed flow is the need to retain nonlinear aspects of the governing equations, since the velocity perturbations are large compared to the ambient speed of sound. This makes analysis of the aerodynamic loading inherently more difficult than subsonic and supersonic flows. Furthermore, as the Mach number is increased, the shock, which is very strong, moves close to the body, while the boundary layer grows rapidly. Therefore, at high Mach numbers, viscous interactions between the outer inviscid flow, the shock, and the boundary layer become significant. ${ }^{1,10,31}$ Also, extreme temperatures are present in both the inviscid flow behind the shock and the boundary layer, due to significant flow compression and viscous dissipation. ${ }^{1,10,31}$ This intense aerodynamic heating can cause dissociation and ionization within the gas, resulting in chemically reacting boundary layers. These important phenomenon are often not accounted for, nor are they generally required, in the analysis of subsonic and supersonic aircraft. Thus, the exact solution to the hypersonic aerodynamic problem can only be obtained by solving the unsteady Navier-Stokes equations, including real gas effects; a task that presents a significant computational challenge.

Aside from these important flow characteristics, another important aspect of hypersonic flight is the tight coupling in hypersonic vehicles between the components that generate lift, propulsion, and vehicle volume. ${ }^{1}$ It is evident from the schematic of the X-43 in Fig. 1 that, unlike subsonic and supersonic aircraft, modern hypersonic vehicles are based on an integrated airframe-propulsion concept; where the entire lower vehicle surface is part of a SCRamjet engine. This integration adds interactions, between the various airframe components, that cannot be neglected in analysis of complete vehicles. ${ }^{11-13}$

\section{Modeling Approaches to Unsteady Hypersonic Aerodynamics}

A challenging aspect of aeroelastic analysis within the hypersonic regime is the accurate and efficient modeling of the unsteady aerodynamic forces. Historically, due to the limited capabilities of computational and experimental facilities, researchers have relied upon a number of approximate unsteady aerodynamic theories for the aeroelastic analysis of hypersonic vehicles. ${ }^{32,33}$ Recently, however, researches have focused on the development and use of high fidelity CFD-based computational aeroelastic tools (CAE); a feasible option due to continual advances in computational capabilities. This section provides a review of both the most common approximate hypersonic aerodynamic modeling approaches, and also research aimed at the fundamental issues of implementing CFD-based unsteady hypersonic aerodynamics for aeroelastic analysis. 


\section{A. Approximate Unsteady Hypersonic Aerodynamics}

The majority of studies on hypersonic aeroelasticity have used either piston theory, ${ }^{34,35}$ or a similar second order theory developed by Van Dyke. ${ }^{36,37}$ However, some other methods, such as unsteady shock-expansion theory ${ }^{38}$ and unsteady Newtonian-Impact theory, ${ }^{39,40}$ have also been employed. Each of these methods assume inviscid hypersonic flow and neglect real gas effects. However, despite these simplifications, such approximate tools have produced sufficiently accurate results in specific cases. ${ }^{7,31,33,41-50}$ Hence, computational efficiency and ease of implementation make these methods attractive for preliminary design and trend type studies of hypersonic configurations. Note that an early review of these methods is available in Ref. [39].

\section{Piston Theory}

Piston theory was developed by Lighthill, ${ }^{34}$ who noted that at high Mach numbers the shock waves and expansion fans on an airfoil form at small angles to the undisturbed flow. This implies that streamwise gradients are small compared to gradients perpendicular to the flow. Furthermore, since velocity components parallel to shock waves and expansion fans are unchanged, velocity components perpendicular to the flow are large compared to disturbances to components parallel to the flow. Consequently, any plane slab of fluid, initially perpendicular to the undisturbed flow, remains so as it is swept downstream and moves in its own plane under the laws of one-dimensional unsteady motion. ${ }^{34,51}$ This realization of the flow is based on Hayes' equivalence principle ${ }^{51}$ which states that "a rotational hypersonic flow on a slender body is equivalent to an unsteady flow in a space having one dimension less".

Using this approximation of the flow, the position of a portion of solid wall, bounding a slab of fluid, moves normal to the flow with the velocity

$$
v_{n}=\frac{\partial Z(x, y, t)}{\partial t}+V_{\infty}\left\{\frac{\partial Z(x, y, t)}{\partial x}+\alpha_{s}\right\}
$$

where

$$
Z(x, y, t)=w_{d}(x, y, z, t)+Z_{s t r}(x, y)
$$

Note that $V_{\infty}$ is the approximate velocity of the slab of fluid as it moves downstream. The above characterization of the flow implies a point-function relationship between the local pressure on a lifting surface and the normal component of fluid velocity produced by the lifting surface motion; ${ }^{35}$ and is equivalent to evaluating the pressure on a piston moving in a one-dimensional channel, as shown in Fig. 4. Assuming the piston generates only simple waves and no changes in entropy, the local pressure is therefore,

$$
\frac{p(x, t)}{p_{\infty}}=\left(1+\frac{\gamma-1}{2} \frac{v_{n}}{a_{\infty}}\right)^{\frac{2 \gamma}{(\gamma-1)}}
$$
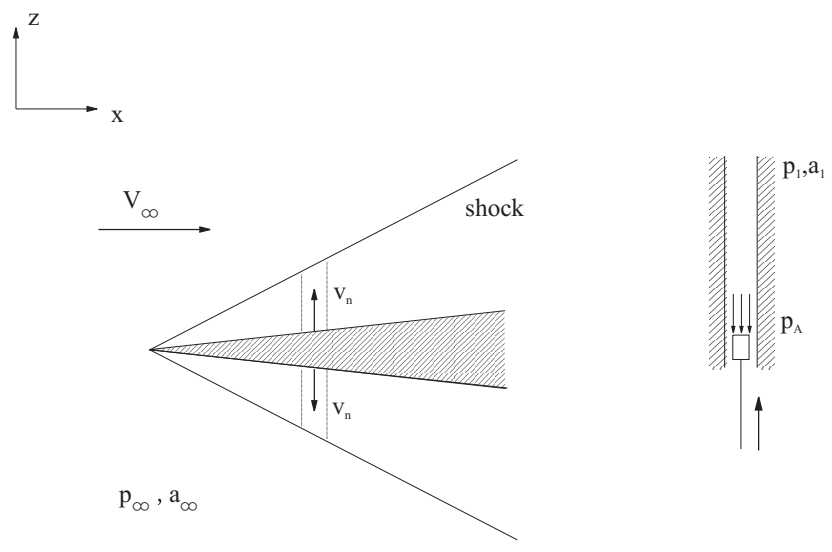

Figure 4. Model for loading on an equivalent piston.[33,50] 
Since this assumes no shock is present, a third order binomial expansion of Eqn. 3 is used, as this yields pressures within $6 \%$ of the simple wave and shock-expansion predictions. ${ }^{34}$ Thus, the piston theory pressure coefficient on an oscillating surface, up to third-order, is given by

$$
C_{p}(x, t)=\frac{2}{M_{\infty}^{2}}\left\{\frac{v_{n}}{a_{\infty}}+\frac{(\gamma+1)}{4}\left(\frac{v_{n}}{a_{\infty}}\right)^{2}+\frac{(\gamma+1)}{12}\left(\frac{v_{n}}{a_{\infty}}\right)^{3}\right\}
$$

Note that this derivation assumes that the free stream Mach number is sufficiently large and the magnitude of the normal component of fluid velocity never exceeds the speed of sound in the undisturbed fluid, ${ }^{34,35}$ i.e.

$$
M_{\infty}^{2} \gg 1 \text { and } M_{\infty}\left[\delta+\left(\frac{w_{d \max }}{c}\right)\left(\frac{\omega_{\max } c}{V_{\infty}}\right)\right]<1
$$

Thus, the accuracy of the piston theory pressure diminishes with increasing Mach number and surface inclination to the free stream.

\section{Van Dyke's Second Order Theory}

As mentioned, Van Dyke's supersonic second order theory ${ }^{36,37}$ is an approximate aerodynamic theory commonly used in supersonic and hypersonic aeroelastic research. Often, due to the similarity of the expressions for pressure, Van Dyke's theory is referred to as piston theory. However, it is important to distinguish between the two theories since they are developed using different approaches. Specifically, Van Dyke's expression for the pressure on an oscillating surface is developed from the nonlinear velocity potential equation, ${ }^{36}$ while Lighthill ${ }^{34}$ uses Eqn. 3.

Assuming irrotational flow, consider the nonlinear velocity potential, where the equation of motion is given by

$$
\begin{aligned}
&\left(a_{l}^{2}-\tilde{\Omega}_{x}^{2}\right) \tilde{\Omega}_{x x}+\left(a_{l}^{2}-\tilde{\Omega}_{y}^{2}\right) \tilde{\Omega}_{y y}+\left(a_{l}^{2}-\tilde{\Omega}_{z}^{2}\right) \tilde{\Omega}_{z z}- \\
& 2 \tilde{\Omega}_{y} \tilde{\Omega}_{z} \tilde{\Omega}_{y z}-2 \tilde{\Omega}_{x} \tilde{\Omega}_{z} \tilde{\Omega}_{x z}-2 \tilde{\Omega}_{x} \tilde{\Omega}_{y} \tilde{\Omega}_{x y}=0
\end{aligned}
$$

The local speed of sound, $a_{l}$, is related to $a_{\infty}$ by

$$
a_{l}^{2}=a_{\infty}^{2}-\frac{\gamma-1}{2}\left(\tilde{\Omega}_{x}^{2}+\tilde{\Omega}_{y}^{2}+\tilde{\Omega}_{z}^{2}-V_{\infty}^{2}\right)
$$

The velocity equation is solved by introducing a perturbation potential into Eqn. 6, i.e.

$$
\tilde{\Omega}=V_{\infty}\left(x+\Phi_{\text {pot }}\right)
$$

such that

$$
\begin{aligned}
\Phi_{p o t, y y}+\Phi_{p o t, z z}-\beta^{2} \Phi_{p o t, x x}= & M_{\infty}^{2}\left\{\frac { \gamma - 1 } { 2 } \left(2 \Phi_{p o t, x}+\Phi_{p o t, x}^{2}+\Phi_{p o t, y}^{2}+\right.\right. \\
& \left.\Phi_{p o t, z}^{2}\right)\left(\Phi_{p o t, x x}+\Phi_{p o t, y y}+\Phi_{p o t, z z}\right)+ \\
& 2 \Phi_{p o t, x} \Phi_{p o t, x x}+\Phi_{p o t, x}^{2} \Phi_{p o t, x x}+\Phi_{p o t, y}^{2} \\
& \Phi_{p o t, y y}+\Phi_{p o t, z}^{2} \Phi_{p o t, z z}+2 \Phi_{p o t, y} \Phi_{p o t, z} \\
& \Phi_{p o t, y z}+2 \Phi_{p o t, x}\left(1+\Phi_{p o t, z}\right) \Phi_{p o t, z x}+ \\
& \left.2\left(1+\Phi_{p o t, x}\right) \Phi_{p o t, y} \Phi_{p o t, x y}\right\}
\end{aligned}
$$

Equation 9 is solved using an iterative procedure. Initially, the first order solution is obtained by neglecting nonlinear terms of $\Phi_{\text {pot }}$ derivatives in Eqn 9. This yields the wave equation, given by

$$
\Phi_{\text {pot }, y y}^{(1)}+\Phi_{\text {pot }, z z}^{(1)}-\beta^{2} \Phi_{\text {pot }, x x}^{(1)}=0
$$

This linearized solution is then substituted into the right-hand side of Eqn. 9 in order to determine the second order solution.

Consider the first-order equation of two-dimensional supersonic flow, given by:

$$
\Phi_{p o t, z z}^{(1)}-\beta^{2} \Phi_{p o t, x x}^{(1)}=0
$$


The general solution is then given by

$$
\Phi_{\text {pot }}^{(1)}(x, z)=a^{(1)}(x-\beta z)+b^{(1)}(x+\beta z)
$$

where $a^{(1)}$ and $b^{(1)}$ are determined from the first order boundary conditions. Substituting Eqn. 12 into Eqn. 9 and neglecting third order and higher terms yields

$$
\Phi_{\text {pot }, z z}^{(2)}-\beta^{2} \Phi_{\text {pot }, x x}^{(2)}=2 M_{\infty}^{2}\left\{\left(\frac{(\gamma+1) M_{\infty}^{2}}{2 \beta^{2}}-1\right) \beta^{2} \Phi_{\text {pot }, x}^{(1)} \Phi_{\text {pot }, x x}^{(1)}+\Phi_{\text {pot }, z}^{(1)} \Phi_{\text {pot }, x z}^{(1)}\right\}
$$

The solution to Eqn. 13 for flow past a single boundary (i.e. one surface of an airfoil) is then given by

$$
\Phi_{\text {pot }}^{(2)}(x, z)=a^{(2)}(x-\beta z)+b^{(2)}(x+\beta z)+\frac{(\gamma+1) M_{\infty}^{4}}{4 \beta^{2}} z \Phi_{\text {pot }, x}^{(1)} \Phi_{\text {pot }, z}^{(1)}
$$

where $a^{(2)}$ and $b^{(2)}$ are determined from the second order boundary conditions. For flow past a curved surface, let the surface shown in Fig. 5 be defined by the following continuous function

$$
z=\bar{\epsilon} Z(x)
$$

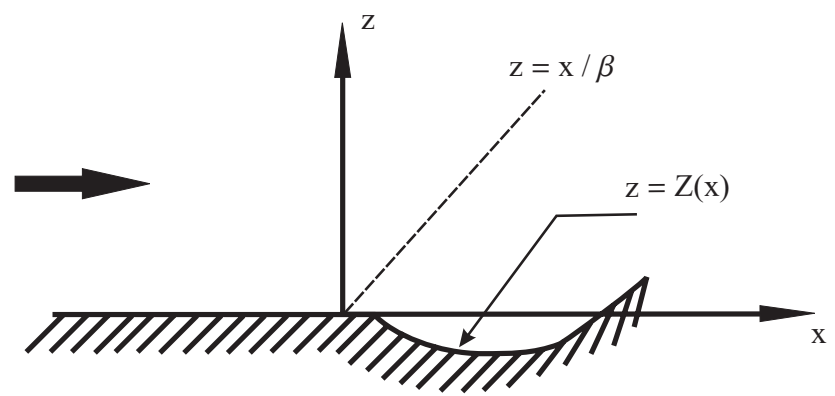

Figure 5. Flow past a curved wall.[36]

where $\bar{\epsilon} \ll 1$ and $Z(x) \sim \mathcal{O}(1)$. In this case, the solution to the first order problem is given by

$$
\Phi_{\text {pot }}^{(1)}=-\bar{\epsilon} \frac{Z(x-\beta z)}{\beta}
$$

and the second-order perturbation potential is thus given by

$$
\begin{array}{r}
\Phi_{\text {pot }}^{(2)}=-\bar{\epsilon} \frac{Z(x-\beta z)}{\beta}-\bar{\epsilon}^{2}\left\{Z(x-\beta z) \frac{\partial Z(x-\beta z)}{\partial(x-\beta z)}+\frac{(\gamma+1) M_{\infty}^{4}}{4 \beta^{3}} z\right. \\
\left(\frac{\partial Z(x-\beta z)}{\partial(x-\beta z)}\right)^{2}+\left(\frac{M_{\infty}^{2}}{2 \beta^{2}}\right)\left(\frac{(\gamma+1) M_{\infty}^{2}}{2 \beta^{2}}-2\right) \\
\left.\int_{0}^{x-\beta z}\left(\frac{\partial Z(\xi)}{\partial \xi}\right)^{2} d \xi\right\}
\end{array}
$$

\section{Determination of the Pressure}

The velocity components of the flow can be determined from the potential field given in Eqn. 17, i.e.

$$
\begin{aligned}
u / V_{\infty} & =\Phi_{\text {pot }, x} \\
v / V_{\infty} & =\Phi_{\text {pot }, y} \\
w / V_{\infty} & =\Phi_{\text {pot }, z}
\end{aligned}
$$


Assuming isentropic flow, the pressure coefficient is then given by:

$$
C_{p}=\frac{2}{\gamma M_{\infty}^{2}}\left\{\left(1+\frac{\gamma-1}{2} M_{\infty}^{2}\left(1-\frac{\left(V_{\infty}+u\right)^{2}+v^{2}+w^{2}}{V_{\infty}^{2}}\right)\right)^{\frac{\gamma}{\gamma-1}}-1\right\}
$$

Thus, using Eqns. 17 - 19, and neglecting terms of $\bar{\epsilon}^{3}$ and higher, the pressure coefficient is given by

$$
C_{p}(x)=\frac{2}{\beta} \bar{\epsilon} \frac{\partial Z(x)}{\partial x}+\frac{(\gamma+1) M_{\infty}^{4}-4 \beta^{2}}{2 \beta^{4}}\left(\bar{\epsilon} \frac{\partial Z(x)}{\partial x}\right)^{2}
$$

If the local steady angle-of-attack, given by $\bar{\epsilon} \frac{\partial Z(x)}{\partial x}$, is replaced by the quasi-steady angle-of-attack, which for thin bodies is given by $\frac{v_{n}}{V_{\infty}}$, the pressure coefficient is ${ }^{37,43}$

$$
C_{p}(x, t)=\frac{2}{M_{\infty}^{2}}\left\{\frac{M_{\infty}}{\beta} \frac{v_{n}}{a_{\infty}}+\frac{M_{\infty}^{4}(\gamma+1)-4 \beta^{2}}{4 \beta^{4}}\left(\frac{v_{n}}{a_{\infty}}\right)^{2}\right\}
$$

Despite the fact that each expression was derived using a different approach, the similarities between the Van Dyke second order quasi-steady pressure coefficient (Eqn. 21) and Lighthill's piston theory expression (Eqn. 4) are evident. Considering only the first and second order terms in Eqn. 4, the main difference is the introduction of $\beta$ in the coefficients of Eqn. 21. However, note that for increasing Mach number, $\beta \rightarrow M_{\infty}$, and the two expressions become equivalent. Therefore, since this expression includes $\beta$, Van Dyke's theory is appropriate for slightly lower Mach numbers than Lighthill's piston theory expression. ${ }^{37,43}$

\section{Unsteady Hypersonic Shock-Expansion Method}

Shock-Expansion theory is a simple method of determining various fluid quantities as the flow passes through shocks and expansion fans that occur on a given shape. ${ }^{52}$ Consider a double-wedge airfoil in steady hypersonic flow, as shown in Fig. 6.

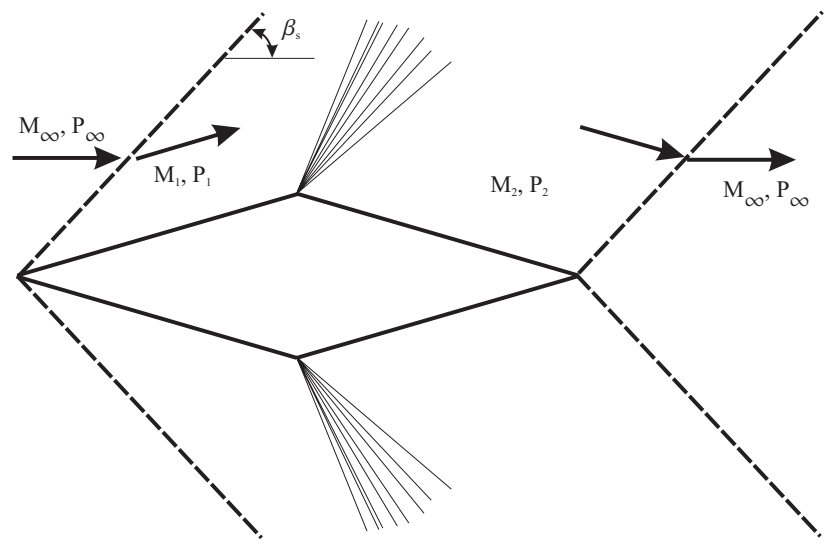

Figure 6. Oblique shocks and expansion fans on a double-wedge airfoil.[1]

Oblique shock waves are present at the leading edge and trailing edge of the airfoil, while expansion fans occur at the mid-chord. The basic procedure of the shock-expansion method ${ }^{1,38}$ is:

1. Calculate the Mach number and pressure behind the oblique shock at the nose using the wedge angle at the nose of the body, i.e. $\theta_{N}$.

2. Assume that the flow is in isentropic expansion along the surface downstream of the nose, where the turning angle of the flow is equal to $\Delta \theta$, i.e.

$$
\Delta \theta(x)=\theta_{l}(x)-\theta_{N}
$$


where $\theta_{l}(x)$ is the local angle of inclination of the body at a given $x$ position along the airfoil.

In order to calculate the Mach number and pressure behind the oblique shock, the oblique shock angle, $\beta_{s}$, must be determined. This is calculated using the following non-linear relation:

$$
\tan \theta_{N}=2 \cot \beta_{s}\left\{\frac{M_{\infty}^{2} \sin ^{2} \beta_{s}-1}{M_{\infty}^{2}\left(\gamma+\cos 2 \beta_{s}\right)+2}\right\}
$$

Since only the normal component of fluid velocity is affected by an oblique shock wave, the Mach number and pressure behind the shock are determined using normal shock relations. Thus, the pressure is given by:

$$
\frac{p_{N}}{p_{\infty}}=\left\{1+\frac{2 \gamma}{\gamma+1}\left(M_{\infty}^{2} \sin ^{2} \beta_{s}-1\right)\right\}
$$

and the Mach number is given by:

$$
M_{N}=\frac{1}{\sin \left(\beta_{s}-\theta_{N}\right)} \sqrt{\frac{M_{\infty}^{2} \sin ^{2} \beta_{s}+\left(\frac{2}{\gamma-1}\right)}{\left(\frac{2}{\gamma-1}\right) M_{\infty}^{2} \sin ^{2} \beta_{s}-1}}
$$

Finally, given the pressure and Mach number behind the oblique shock, the pressure at any point along the surface of a body is determined using the Prandtl-Meyer expansion formula, i.e.

$$
p(x)=p_{N}\left\{1+\frac{\gamma-1}{2} M_{N} \Delta \theta(x)\right\}^{\frac{2 \gamma}{\gamma-1}}
$$

In Ref. [38], the extension of these relations to unsteady flow are discussed. This is accomplished using a similar approach to that of Refs. [37,43], where the expression for the steady pressure was extended to an unsteady pressure by replacing the steady inclination angle of the airfoil surface in Eqns. 23 through 26 with the quasi-steady inclination, i.e.

$$
\begin{aligned}
\theta_{N}(t) & =\tan ^{-1} \frac{v_{n}\left(x_{N}, t\right)}{V_{\infty}} \\
\theta_{l}(x, t) & =\tan ^{-1} \frac{v_{n}(x, t)}{V_{\infty}}
\end{aligned}
$$

Combining Eqns. 22 through 27, the pressure coefficient for unsteady hypersonic shock-expansion theory is given by

$$
C_{p}(x, t)=\frac{2}{\gamma M_{\infty}^{2}}\left\{\left(\frac{p_{N}}{p_{\infty}}\right)\left(1+\frac{\gamma-1}{2} M_{N} \Delta \theta(x, t)\right)^{\frac{2 \gamma}{\gamma-1}}-1\right\}
$$

Note that when using this approach, one assumes that the body has a sharp leading edge with an attached shock.

\section{Unsteady Newtonian-Impact Theory}

In general, the distance between the shock and surface of a vehicle diminishes as the Mach number of the flow increases. ${ }^{1,31}$ For a fluid moving over relatively simple shapes, at a sufficiently high Mach number (i.e. $M_{\infty} \gg 1$ ), one can assume that the speed and direction of the gas particles in the freestream remain unchanged until they strike the windward surface of the vehicle. ${ }^{1,10,31}$ Using this assumption, and ignoring intermolecular forces, it is evident that the normal component of momentum of the impinging fluid particle is lost, while the tangential component of momentum is conserved. ${ }^{1,10,31}$ Consider the surface in Fig. 7, which is exposed to hypersonic flow.

Using this model, the change in momentum for a constant-area streamtube normal to the surface is given by $^{31}$

$$
\left(\rho_{\infty} \mathbf{U}_{\infty} \cdot \hat{\mathbf{n}}\right)\left(\mathbf{U}_{\infty}-\mathbf{U}_{w}\right)=\left(p_{w}-p_{\infty}\right) \hat{\mathbf{n}}
$$




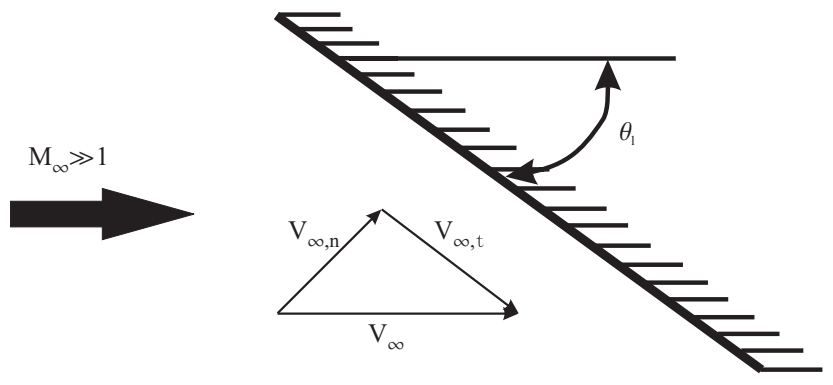

Figure 7. Newtonian flow model.[10]

Since the normal component of momentum is zero at the body surface, this reduces to

$$
p_{w}-p_{\infty}=\rho_{\infty}\left(\mathbf{U}_{\infty} \cdot \hat{\mathbf{n}}\right)^{2}
$$

and therefore the pressure coefficient is given by

$$
C_{p}=2\left(\frac{\mathbf{U}_{\infty} \cdot \hat{\mathbf{n}}}{\left|\mathbf{U}_{\infty}\right|}\right)^{2}
$$

For a surface at an inclination $\theta_{l}$ to the flow

$$
\mathbf{U}_{\infty} \cdot \hat{\mathbf{n}}=-\left|\mathbf{U}_{\infty}\right| \sin \theta_{l}
$$

Thus, the pressure coefficient using Newtonian-Impact theory is given by

$$
C_{p}=2 \sin ^{2} \theta_{l}
$$

Note that for

$$
\mathbf{U}_{\infty} \cdot \hat{\mathbf{n}} \geq 0
$$

the flow is in the "aerodynamic shadow". In this region, $C_{p}$ is assumed to be zero.

It has been shown that Eqn. 33 provides a reasonable approximation for the flow over a generic object when $M_{\infty} \rightarrow \infty$ and $\gamma \rightarrow 1_{1}^{1,10,31}$ This corresponds to a shock wave outlining the body surface, and an infinitesimal shock layer between the body and the shock. It has been found, however, that the theory will yield sufficiently accurate results at somewhat lower Mach numbers when Eqn. 33 is replaced by,

$$
C_{p}=\left(C_{p}\right)_{\max } \sin ^{2} \theta_{l}
$$

where

$$
\left(C_{p}\right)_{\max }=\frac{2}{\gamma M_{\infty}^{2}}\left(\left(\frac{(\gamma+1)^{2} M_{\infty}^{2}}{4 \gamma M_{\infty}^{2}-2(\gamma-1)}\right)^{\frac{\gamma}{(\gamma-1)}}\left(\frac{1-\gamma+2 \gamma M_{\infty}^{2}}{\gamma+1}\right)-1\right)
$$

which corresponds to the pressure coefficient at the stagnation point of the body. The expression in Eqn. 35 is referred to as modified Newtonian-Impact theory. ${ }^{1,10}$

The unsteady Newtonian aerodynamic expression is obtained by replacing the steady inclination angle with the quasi-steady angle, ${ }^{14}$

$$
C_{p}(x, t)=\left(C_{p}\right)_{\max } \sin ^{2}\left\{\tan ^{-1}\left(\frac{v_{n}}{V_{\infty}}\right)\right\}
$$

Note that this theory is referred to as Newtonian-Impact theory since it is similar in character to the model described by Sir Isaac Newton in the seventeenth century. ${ }^{1}$ Unsteady Newtonian-Impact aerodynamics provide a simple manner to handle extremely high Mach number flows, and/or portions of the flow largely inclined to the surface of a body. ${ }^{14,39,51,53}$ 


\section{Modeling Viscous Effects With an Effective Shape}

As noted in Section II, viscous interactions, where the boundary layer displaces the outer inviscid flow causing a given body shape to appear much thicker, become significant in hypersonic flow. ${ }^{1,10,31}$ This apparent thickness influences both the surface pressure distribution and the vehicle aeroelastic stability. ${ }^{33,41,42,46}$ In an attempt to account for this effect in the aeroelastic computations, the a static boundary layer displacement thickness can be computed and added to the surface of a given body. ${ }^{33,42,46}$

Two methods have been used by researchers to compute the static boundary layer displacement. The first method utilizes semi-empirical, compressible flat plate boundary layer relations to generate the effective shape. ${ }^{33,42,46}$ Such an approach was considered in Ref. [42], where a flat-plate boundary layer thickness was used to modify the shape of airfoil sections in order to improve correlation of theoretical results with experiments. The second approach uses steady pressure data generated by solving the steady Navier-Stokes equations. ${ }^{33,46}$

\section{Semi-Empirical Boundary Layer Displacement Thickness Equations}

The semi-empirical relation used in Ref. [42] is given in Cox and Crabtree $^{54}$ as:

$$
Z_{f p}(x)=\frac{\gamma-1}{\gamma+1}\left(0.664+1.73 \frac{T_{W}}{T_{0}}\right) M_{\infty}^{2} \sqrt{\frac{C_{w}}{R e_{x}}} x
$$

Note that Eqn. 38 is based on the assumption of laminar flow with weak viscous interactions. It is further manipulated by introducing additional assumptions. First, assuming $\operatorname{Pr} \approx 1$ and an adiabatic wall condition, i.e. $T_{W}=T_{A W}$, implies

$$
\frac{T_{W}}{T_{0}} \approx 1
$$

Next, the Chapman-Rubesin coefficient, $C_{w}$, can be computed using

$$
C_{w}=\frac{\mu_{w}}{\mu_{\infty}} \frac{T_{\infty}}{T_{W}}
$$

where $T_{W}$ is given by

$$
T_{W}=T_{\infty}\left(1+\frac{\gamma-1}{2} M_{\infty}^{2}\right)
$$

and $\mu_{w}$ is given by Sutherland's Law, i.e.

$$
\mu_{w}=1.458 \times 10^{-6} \frac{T_{W}^{1.5}}{T_{W}+110.4}
$$

Note that Eqn. 42 is dimensional, and assumes $T_{W}$ is in units Kelvin, resulting in $\mu_{w}$ in units $\frac{\mathrm{kg}}{\mathrm{ms}}$. Using Eqns. 38 - 42 in conjunction with free stream properties of the fluid, and the position $x$ along the airfoil, yields the approximate effective shape of the airfoil due to a viscous boundary layer, i.e.

$$
Z_{e f f}(x)=Z_{f p}(x)+Z_{s t r}(x)
$$

Boundary Layer Displacement Thickness Approximated From the Steady CFD Pressure Coefficient

The amount of viscous interaction between the outer inviscid flow and flow within the boundary layer can vary between strong and weak, depending on position along the surface of a body. ${ }^{1}$ Furthermore, boundary layers in hypersonic flow are often characterized by both laminar and turbulent regions, with a specific transition point along the body. ${ }^{10}$ Both of these issues degrade the accuracy of the effective shape given by Eqn. 38, for certain regions of an aerodynamic surface. ${ }^{33}$ The accuracy in calculating the displacement thickness can be improved, however, by using a CFD solution of the steady Navier-Stokes equations. ${ }^{33,46}$ In such an approach, the effective shape correction is obtained from the steady pressure distribution by setting 
up a point function relation between the pressure and the surface of the body. Since piston theory provides such a relation, it is a convenient choice for this approach. ${ }^{33,46}$

The steady component of the piston theory pressure coefficient is obtained from Eqn. 4 by neglecting all time dependent terms. For zero angle-of-attack, this is given by:

$$
C_{p P T}(x)=\frac{2}{M_{\infty}}\left\{\frac{d Z_{\text {eff }}}{d x}+\frac{(\gamma+1)}{4} M_{\infty}\left(\frac{d Z_{\text {eff }}}{d x}\right)^{2}+\frac{(\gamma+1)}{12} M_{\infty}^{2}\left(\frac{d Z_{\text {eff }}}{d x}\right)^{3}\right\}
$$

Equating the steady CFD Navier-Stokes coefficient of pressure with Eqn. 44 yields a third order polynomial for $\frac{d Z_{e f f}}{d x}$

$$
C_{p P T}(x)-C_{p N S}(x)=0
$$

Solving this equation at each surface grid point results in two complex roots, and one real root that represents the slope of the effective airfoil shape at that grid point. The complete effective shape, $Z_{\text {eff }}(x)$,

can then be obtained from this slope, $\frac{d Z_{\text {eff }}}{d x}$, by integrating along the length of the airfoil, and assuming zero displacement thickness at the leading edge.

\section{B. CFD-based Unsteady Hypersonic Aerodynamics}

As computational power has increased in recent years, CFD solutions to the Euler and Navier-Stokes equations have emerged as a viable alternative, and significant improvement in many cases, to experimental testing and approximate aerodynamic theories. Recently, a number of studies have been conducted that investigate the use of CFD-based unsteady aerodynamics in the aeroelastic/aerothermoelastic analysis of hypersonic vehicles. ${ }^{7,33,41,44-50}$

There are three main issues with utilizing CFD-based aerodynamics in an aeroelastic analysis, namely: 1) fluid-structural coupling, 2) spatial accuracy, and 3) temporal accuracy. Since careful consideration of these issues is required to minimize the relatively high cost of CFD-based aeroelastic analysis, each is discussed next. Note that these are general issues of any CFD-based aeroelastic analysis, however, where appropriate, the scope of the present discussion is limited to the consideration of hypersonic flow only.

\section{Computational Methods for Fluid-Structure Coupling}

Prediction of the dynamic response of a flexible structure in a fluid requires the simultaneous solutions of the equations of motion of the structure and the fluid. In order to impose the kinematic boundary conditions on the fluid mesh at the new time step, the location and velocity of the fluid-structure boundary must first be known. This requires the solution of the entire system of equations for the structure, a task that cannot be carried out until the current surface pressure is known, which depends on the solution of the fluid domain and thus also on the unknown boundary conditions during the current time step. In addition, the discretized model of the structure uses a Lagrangian approach by following a point located on the structure over time, while the discretized model of the fluid uses an Eulerian approach by computing the flow quantities at a specific location in space over time. Therefore, accurate coupling of the two systems is a fairly complicated endeavor.

A straight forward approach to the solution of the coupled fluid-structure system requires changing the fluid grid at each time-step, which is computationally very expensive. Therefore, several different approaches have emerged as alternatives to partial regridding in transient aeroelastic computations, among them being the space-time formulation, ${ }^{55-57}$ the Arbitrary/Mixed Eulerian-Lagrangian formulation, ${ }^{58,59}$ the multiplefield formulation, ${ }^{60-67}$ the transpiration method, ${ }^{7,68}$ and the exponential decay method, ${ }^{66,69}$ Each of these methods are briefly reviewed next.

Space-Time Method

The Space-Time approach ${ }^{55-57}$ attempts to discretize the space-time domain using finite elements, i.e. the finite element mesh covers the complete space-time domain. The variational formulation of the problem is written over the associated space-time domain. While the motion of the boundary is explicitly unknown, the location of the boundary nodes at the end of a time-step are related to the other unknowns (i.e. velocity, displacement) at the boundary of the spatial domain. The solution to this space-time variational problem 
includes the complete motion of the spatial domain within the time-period of interest. Another advantage of this approach is that it allows the use of spatially local time steps within a temporally accurate formulation. ${ }^{57}$ When applying this method to aeroelastic problems, a simultaneous solution of the fluid and the structure over the combined space-time domains is the natural extension. However, in such a case, the solution and variational function spaces needs to include functions which are discontinuous across the interface.

\section{Arbitrary/Mixed Eulerian-Lagrangian Formulation}

The Lagrangian approach applied to fluid flows provides a clear delineation of interfaces and well-resolved details of the flow, but is limited by its inability to cope with large motions of particles as seen in high speed flows. On the contrary, in the Eulerian formulation, large motions can be handled with relative ease, but generally at the expense of precise feature definition and resolution of detail. Both the Lagrangian and Eulerian approaches present advantages and drawbacks, and the Arbitrary Lagrangian-Eulerian (ALE) method attempts to combine the best features of both descriptions. ${ }^{58}$ This is done by considering a reference frame which moves at a non-zero velocity, and is also different from the particle velocity. The conservation laws are then rederived in this frame. However, this method is primarily intended to simplify the treatment of the boundary nodes, or to provide a rezoning capability for the fluid mesh. In the Mixed LagrangianEulerian method, ${ }^{59}$ the governing equations for both the fluid and the structure are formulated in integral conservation form based on the same Lagrangian-Eulerian description. At the fluid-structure boundary, there is a switch from the Lagrangian-Eulerian description to a Lagrangian description, and from Cartesian to generalized coordinates. The entire fluid-structure continuum is treated as one continuum dynamics problem, while allowing for different discretizations in the two domains. The same numerical integration algorithm can then be used throughout all elements in the field meshes covering the fluid-structure system.

\section{Multiple Field Formulation}

In the Multiple Field formulation, ${ }^{60}$ the moving mesh is viewed as a psuedostructural system with its own dynamics and thus the coupled transient aeroelastic problem is formulated as a three-field problem: the fluid, the structure and the dynamic mesh. In Ref. [61], this approach was implemented using a spring analogy; where the edges of each element are represented as springs, with stiffnesses inversely proportional to the length of the edge. Grid points on the outer boundary of the mesh are held fixed, and the instantaneous locations of points on the inner boundary (body) are prescribed by the body motion. At each time-step, the static equilibrium equations along the coordinate axes are solved iteratively at each interior node of the grid for its displacements. This is accompanied by a predictor-corrector method, which first predicts the displacements according to a linear extrapolation and then corrects these displacements using several Jacobi iterations of the static equilibrium equations. It was noted by Batina ${ }^{61}$ that a geometric conservation law, relating the change in the area/volume of a cell to the area/volume swept by the boundary of the cell, must be satisfied in order to avoid errors induced by the moving mesh. It is important to mention that the spring analogy approach of Batina ${ }^{61}$ was developed for unstructured meshes. In Ref. [64], the method was modified for use in multi-block structured grids. Furthermore, the problem of grid collapse around convex surfaces was addressed by selectively increasing/decreasing spring stiffness based on surface curvature. ${ }^{64}$

The finite macro-element method is another example of an elasticity based dynamic mesh approach. ${ }^{66,67}$ In this approach, the fluid domain is divided into "Macro-Elements", which are created from a subset of fluid grid points. The deformation of the mesh is then solved using a finite element approach, where the stiffness of the elements is varied according to distance to the nearest surface (stiffness increases toward the surface). Once the displacements of the "Macro-Elements" are determined, the updated locations of intervening mesh points are placed according to a Transfinite Interpolation (TFI) step; an algebraic scheme ${ }^{70}$ that efficiently maps grid displacements from one block face to another using polynomial functions.

\section{Transpiration Approach}

The transpiration method ${ }^{68}$ is a means by which to "trick" the flow solver into seeing a deflection in the mesh that is not actually there. If a change in the surface normal is known, from a structural dynamic solver for example, then this change in the normal surface can be applied directly to the existing CFD grid through a slight modification of the existing surface normals. With transpiration, the nodes affected by a surface deflection simply require a modification of the existing surface normals. Even though the surface is not actually deflected, the flow solver sees the deflected normal at the corresponding nodal locations. 
The exponential decay approach ${ }^{66,69}$ is an algebraic mesh deformation method in which surface movement is transmitted into the mesh interior using an exponential decay function. The motion of selected slave points, chosen across a grid at constant index intervals, is tied to the motion of the nearest surface (or master) point. The exponential decay function uses distance between the slave and nearest surface point so that motion of the surface is transmitted nearly undiminished to nearby slave points. Intervening mesh points on block faces are updated using TFI. Once the intermediate mesh between slave points is updated on block faces, block interiors are updated using a volume TFI step.

\section{Grid Construction for Computational Aeroelasticity in Hypersonic Flow}

In CFD computations, fluid mesh "quality" is critical for the accurate prediction of the aerodynamic loads present on a body. The development of a high quality mesh, however, becomes a challenging task for threedimensional configurations since the grid resolution required for accurate flow computations often places an excessive burden on computational resources. The ideal distribution of cells around a body in hypersonic flow was investigated in Ref. [47], in order to ensure accurate flow predictions at reasonable computational costs. In this study ${ }^{47}$ a grid convergence analysis was carried out for steady hypersonic flow past the low-aspect ratio wing shown in Fig. 8.

The first part of the analysis ${ }^{47}$ investigated the ideal distribution of cells around the wing. This was accomplished by computing the inviscid pressure distribution over the wing surface, using the four different candidate grid configurations illustrated in Figs. 9 and 10. Note that the computational domain over the forward portion of the wing in MESH2-MESH4 was limited to a distance just beyond the shock that forms at $M_{\infty}=5.0$, since only hypersonic flows were considered. ${ }^{33,47}$

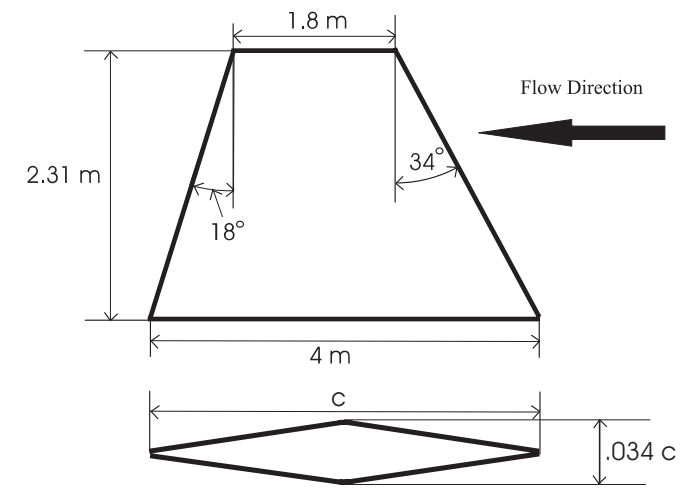

Figure 8. Planform and cross-sectional views of the low-aspect ratio wing used in Ref. [47].

The inviscid pressure distributions for each test case, at a cross-section located at $75 \%$ span of the wing, is shown in Fig. 11 for both a moderate $(M=5.0)$ and high $(M=16.0)$ Mach number. The importance of distributing the cells appropriately is clearly illustrated. For the geometry shown in Fig. 8, the pressure at a cross-section of the wing should be a step function, with the pressure constant from leading edge to midchord, and mid-chord to trailing edge; with the discontinuity at mid-chord. However, as illustrated in Fig. 11, the surface pressure predicted using MESH1 was non-uniform over the forward and aft sections. This indicates that neither the shock nor the expansion fan is captured well, and the discontinuity in pressure is distributed over a large number of streamwise grid points. Furthermore, while MESH2 - MESH4 all capture the leading edge shock, only MESH4 correctly computes a sharp change in pressure at mid-chord. This is particularly visible at $M=16.0$. Based on these results, it was concluded ${ }^{47}$ that MESH4, with the majority of cells concentrated near the wing surface, produced the most accurate representation of the flow characteristics around the wing. This is necessary due to the large velocity gradients present at the leading/trailing edges and at mid-chord. As noted in Ref. [47], a convenient feature of this requirement is the ability of the grid to also resolve the boundary layer in Navier-Stokes computations. Thus in hypersonic flow, both Euler and Navier-Stokes computations should be performed using similar cell distributions. ${ }^{33,47}$ 


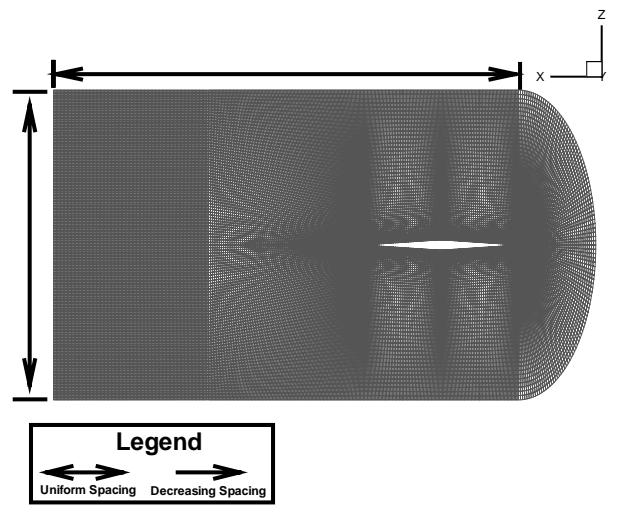

(a) Root plane of MESH1.

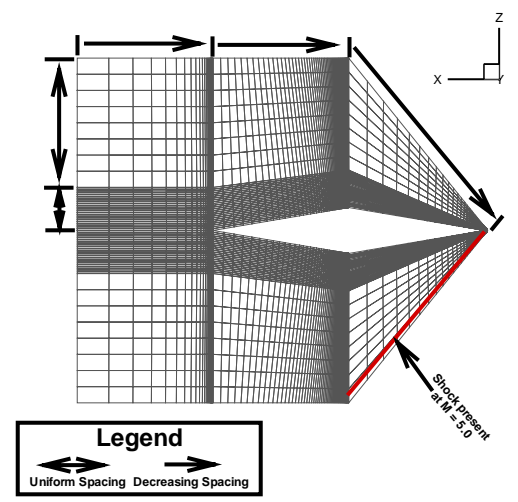

(c) Root plane of MESH3.

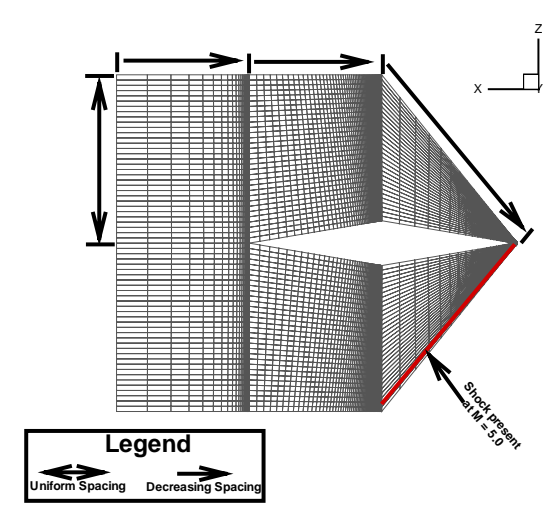

(b) Root plane of MESH2.

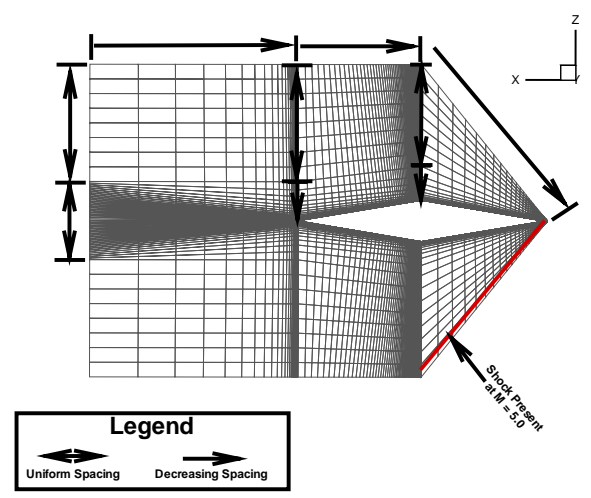

(d) Root plane of MESH4.

Figure 9. Comparison of several different types of hypersonic mesh configurations. Two-sided arrows designate a region of uniform spacing, while one-sided arrows indicate a direction of decreasing cell spacing. [47]

Mach contour plots, shown in Fig. 12, demonstrate the ability of a reduced domain to utilize all of the computational cells. Furthermore, Fig. 12 also illustrates the computation of both the inviscid and viscous flow characteristics with the same grid configuration.

The effect of grid resolution on the accuracy of the flow solution was also investigated in Ref. [47]. A comparison of the force coefficients with increasing grid resolution, generated using both Euler and NavierStokes aerodynamics, are provided in Tables 1 and 2. It is evident that the lift and moment coefficients were relatively insensitive to grid resolution for both the Euler and Navier-Stokes computations. In contrast, the Navier-Stokes coefficient of drag required significant grid refinement to converge. ${ }^{33,47}$ Note, however, that flutter of the wing was found to be relatively insensitive to grid resolution, even when the unsteady aerodynamics were computed using the Navier-Stokes computations. ${ }^{33,49}$ This result will be discussed in more detail in a latter section of this paper.

Finally, it is important to mention that the reduced domain for a grid, as discussed in Ref. [47], is only useful for cases where the aeroelastic steady-state is nearly undeflected from the initial position (e.g. zero angle-of-attack for the wing considered in [47]). For an aeroelastic system where there is considerable deflection at the aeroelastic steady-state, the outer boundary of the grid must be expanded to accommodate the surface deflection. In such a case, however, only a minimal number of cells outside the flow domain of influence (i.e. outside the shock wave) are required. 


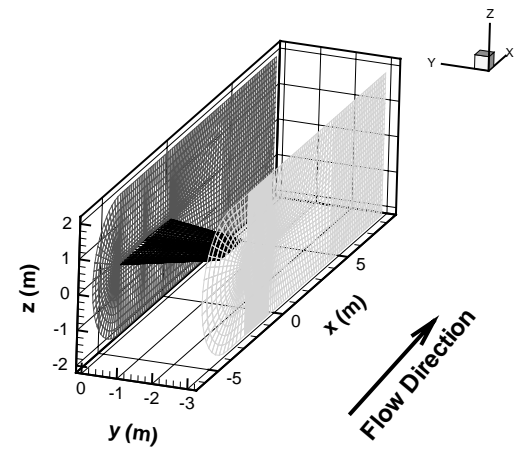

(a) $\operatorname{MESH} 1\left(4.0 \times 10^{6}\right.$ cells $)$.

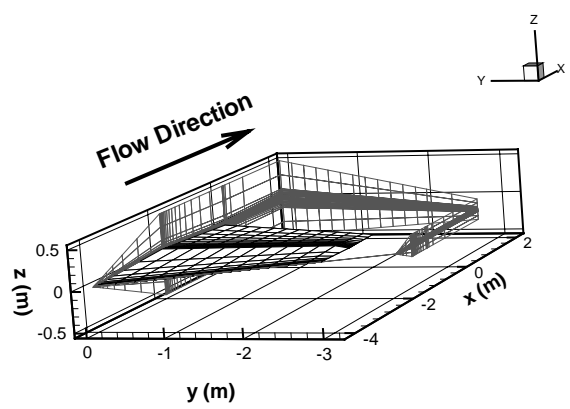

(b) MESH2 - MESH4 $\left(0.63 \times 10^{6}\right.$ cells $)$.

Figure 10. Computational domains of the candidate mesh configurations.[47]

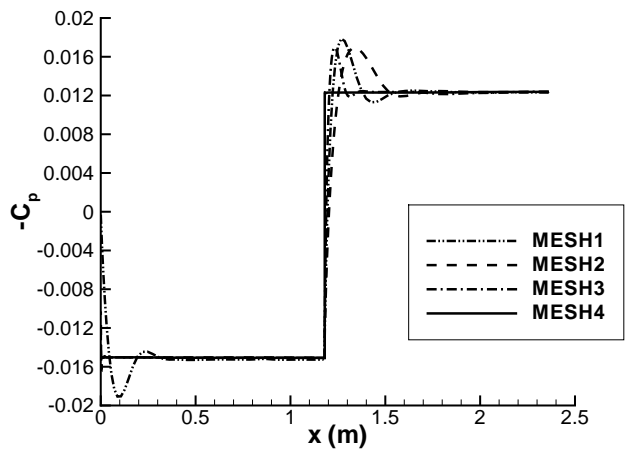

(a) Mach 5, 40,000 ft.

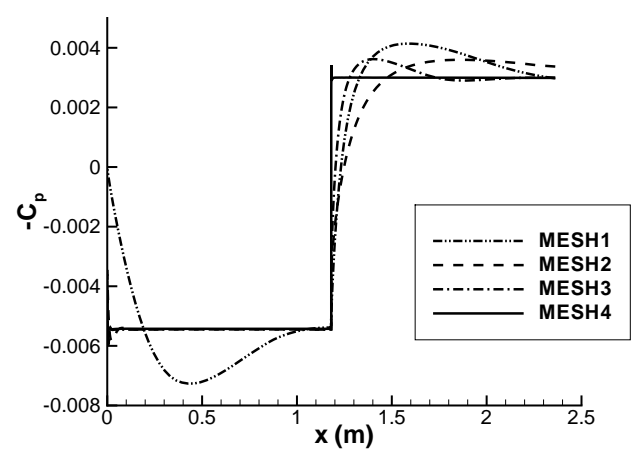

(b) Mach 16, 40,000 ft.

Figure 11. Values of $C_{p}$ from 4 different grids using Euler aerodynamics, for a section located at the $75 \%$ span of the low-aspect ratio wing. [47]

Table 1. Effect of grid resolution on the accuracy of $C_{L}$ and $C_{M}$ computations using Euler aerodynamics[47].

\begin{tabular}{c|cc|cc}
\hline \hline \multicolumn{3}{c}{$C_{L}$} & \multicolumn{2}{c}{$C_{M}$} \\
\hline \# of Cells & $M=5.0$ & $M=16.0$ & $M=5.0$ & $M=16.0$ \\
\hline $0.08 \times 10^{6}$ & $0.70 \%$ & $3.09 \%$ & $1.84 \%$ & $3.16 \%$ \\
$0.27 \times 10^{6}$ & $0.74 \%$ & $1.53 \%$ & $0.96 \%$ & $1.84 \%$ \\
$0.63 \times 10^{6}$ & $0.67 \%$ & $1.11 \%$ & $0.10 \%$ & $1.29 \%$ \\
$2.1 \times 10^{6}$ & $0.23 \%$ & $0.44 \%$ & $0.24 \%$ & $0.43 \%$ \\
$5.1 \times 10^{6}$ & $\mathrm{~N} / \mathrm{A}$ & $\mathrm{N} / \mathrm{A}$ & $\mathrm{N} / \mathrm{A}$ & $\mathrm{N} / \mathrm{A}$ \\
\hline \hline
\end{tabular}




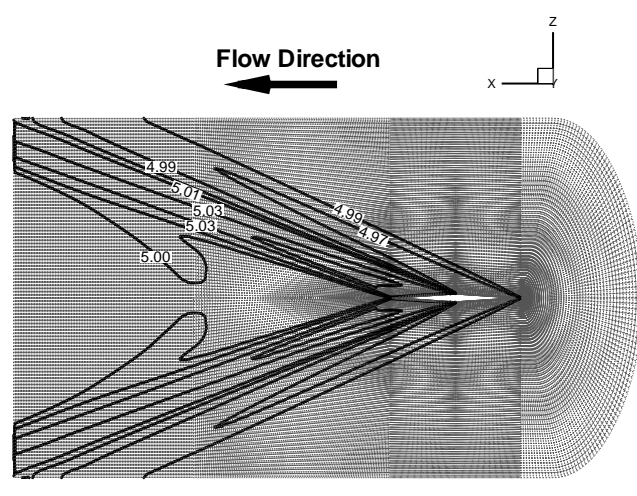

(a) MESH1, Euler Aerodynamics

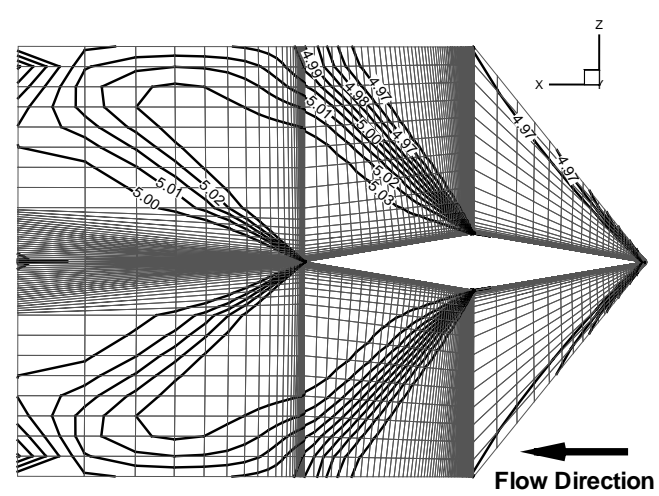

(b) MESH4, Euler Aerodynamics

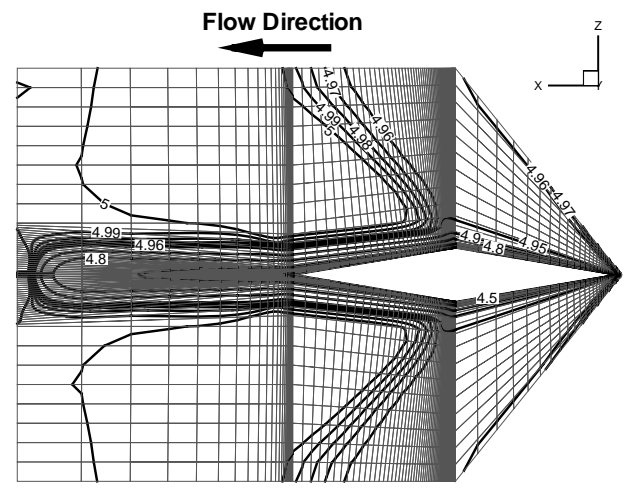

(c) MESH4, Navier-Stokes Aerodynamics

Figure 12. Mach contours of the flow at a section located at $75 \%$ span of a low-aspect ratio wing; $M=5.0$, $40,000 \mathrm{ft}$. Note that the $\mathrm{z}$ dimension is scaled.[47]

Table 2. Effect of grid resolution on the accuracy of $C_{L}, C_{M}$, and $C_{D}$ computations using Navier-Stokes aerodynamics[47].

\begin{tabular}{c|cc|cc|cc}
\hline \hline \multicolumn{3}{c}{$C_{L}$} & \multicolumn{2}{c}{$C_{M}$} & \multicolumn{2}{c}{$C_{D}$} \\
\hline \# of Cells & $M=5.0$ & $M=16.0$ & $M=5.0$ & $M=16.0$ & $M=5.0$ & $M=16.0$ \\
\hline $0.08 \times 10^{6}$ & $0.82 \%$ & $6.96 \%$ & $7.15 \%$ & $4.44 \%$ & $21.75 \%$ & $96.23 \%$ \\
$0.27 \times 10^{6}$ & $1.28 \%$ & $4.41 \%$ & $6.23 \%$ & $4.86 \%$ & $15.30 \%$ & $51.11 \%$ \\
$0.63 \times 10^{6}$ & $1.14 \%$ & $3.85 \%$ & $5.53 \%$ & $3.85 \%$ & $11.48 \%$ & $24.96 \%$ \\
$2.1 \times 10^{6}$ & $0.29 \%$ & $1.44 \%$ & $0.32 \%$ & $1.44 \%$ & $2.05 \%$ & $8.76 \%$ \\
$5.1 \times 10^{6}$ & $\mathrm{~N} / \mathrm{A}$ & $\mathrm{N} / \mathrm{A}$ & $\mathrm{N} / \mathrm{A}$ & $\mathrm{N} / \mathrm{A}$ & $\mathrm{N} / \mathrm{A}$ & $\mathrm{N} / \mathrm{A}$ \\
\hline \hline
\end{tabular}

\section{Temporal Accuracy in Hypersonic Aeroelastic Computations}

The optimal time-step for CAE analysis is ultimately based on a trade-off between accuracy and the demands on computational resources. ${ }^{46}$ A numerical study of this trade-off, with hypersonic flow conditions, was implemented in Ref. [46] by computing the unsteady lift, moment and drag coefficients $\left(C_{L}(t), C_{M y}(t)\right.$, and 
$\left.C_{D}(t)\right)$ due to prescribed motion of a double-wedge airfoil. The analysis was carried out using the NASA Langley CFL3D code; $;^{71}$ with computations at 10,20 and $30 \mathrm{~Hz}$, a forced pitch motion at $1^{\circ}$ and $2^{\circ}$, and altitudes of 50,000, 70,000, and 100,000 feet. Results corresponding to an altitude of 70,000 feet, $M_{\infty}=10.0$, and a maximum rotation of $2^{\circ}$ at $20 \mathrm{~Hz}$, are shown in Fig. 13. From the analysis, it was determined that the drag coefficient exhibited the most sensitivity to the time step in terms of phase errors in the solution. Since phase errors in $C_{L}(t)$ and $C_{M y}(t)$ can have a significant effect in the stability of the problem, it was concluded that the drag coefficient was the best indicator of convergence. A suggested guide for the time step as a function of operating conditions is given in Table 3. It is apparent from these results that increasing the Mach number and the altitude requires a decrease in time step. Note that errors in phase and magnitude were not found to be strongly affected by the frequency or magnitude of the oscillations at each operating condition. ${ }^{46}$

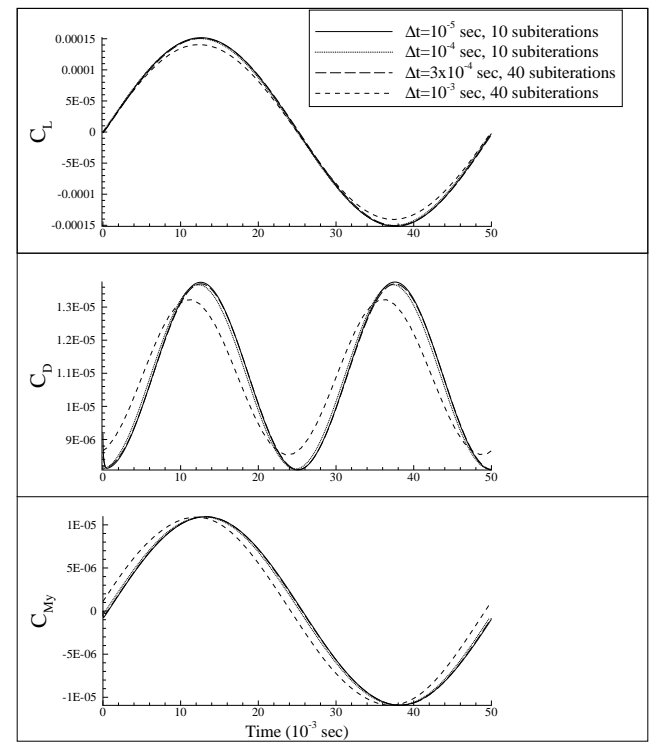

Figure 13. Results for forced pitching motion at Mach 10 and 70,000 feet, with a maximum pitch angle of $2^{\circ}$ at $20 \mathrm{~Hz} \cdot[46]$

Table 3. Suggested timestep sizes and corresponding number of sub-iterations for aeroelastic simulations.[46]

\begin{tabular}{cccc}
\hline \hline Altitude (feet) & Mach no. & Time-step size (sec) & No. of sub-iterations \\
\hline 50,000 & 5.0 & 0.001 & 10 \\
70,000 & 10.0 & 0.0003 & 40 \\
100,000 & 10.0 & 0.0001 & 40 \\
\hline \hline
\end{tabular}

The CFL3D code, used in Ref. [46], steps forward in time using an implicit approximate-factorization method. While computationally efficient, this method leads to linearization and factorization errors at each time step. ${ }^{71}$ As evident from Table 3, a sub-iteration scheme is necessary in order to reduce these errors and achieve the desired accuracy. Thus, a subsequent study ${ }^{49}$ was conducted on the sensitivity of the aeroelastic behavior of a low-aspect ratio wing, shown in Fig. 8, to temporal input parameters governing the subiteration schemes used in CFL3D. Specifically, the "pseudo time sub-iteration" method was examined. This method is implemented by specifying both the number of sub-iterations, and the "pseudo" time step size. The latter is set by the input parameter $C F L_{\tau}$. Note that in general, decreasing the global time step reduces the number of sub-iterations required to achieve the desired temporal accuracy, while increasing the grid 
refinement increases the number of sub-iterations required. ${ }^{71}$

The effect of sub-iterations and $C F L_{\tau}$ on the hypersonic aeroelastic behavior of the low-aspect ratio wing is illustrated in Fig. 14. Note that these results were generated at 40,000 feet, for $M_{\infty}=12.0$. This operating condition was predicted, via preliminary analysis, to be relatively close to, but not above, the flutter boundary of the wing. Also note that the global time step was set to $\Delta t=0.0025$ secs. It is clear from Fig. 14 that system damping varies with an increasing number of sub-iterations, while the frequencies of all the modes remain relatively constant. In particular, the damping of the first and second modes, which are important for flutter analysis, are significantly affected by the number of sub-iterations. Specifically, the damping of the first and second modes switch in magnitude as the number of sub-iterations is increased. It was concluded, that for the configuration and operating conditions considered, at least 20 - 35 sub-iterations $\left(C F L_{\tau}=5.0\right)$ were required for solving the Euler equations, while 50 sub-iterations $\left(C F L_{\tau}=5.0\right)$ were required for the Navier-Stokes equations. ${ }^{33,49}$

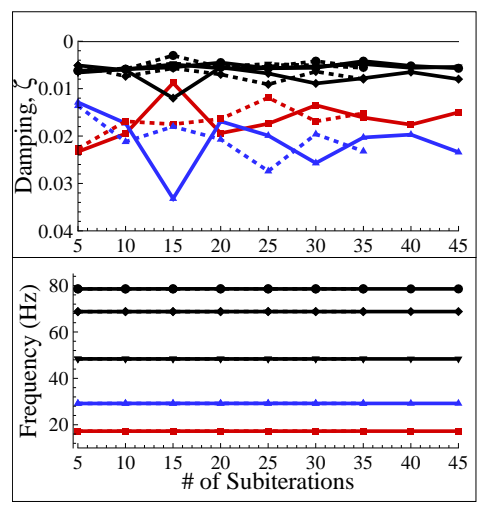

(a) Euler

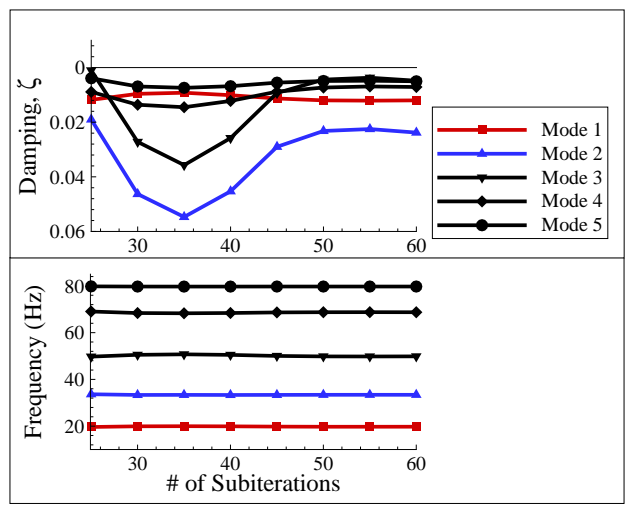

(b) Navier-Stokes $\left(C F L_{\tau}=5.0\right)$

Figure 14. Effect of increasing sub-iterations and $C F L_{\tau}$ on the aeroelastic behavior of the low-aspect ratio wing at $40,000 \mathrm{ft} .[49]$

\section{Panel Flutter}

Panel flutter is an aeroelastic instability that occurs in a small localized region, and effects structural components, such as a skin panel, on the surface of a supersonic or hypersonic vehicle. The combination of minimum weight requirements and high speed flow, which generates intense aerodynamic heating and unsteady surface pressures, results in thin panels subject to substantial in-plane and out-of-plane loads.

Since this problem is relatively easy to simulate, panel flutter has been studied extensively. Thus, a complete survey of research conducted on panel flutter is beyond the scope of this paper. A partial review is useful because the methods that have been employed, as well as several results, have a connection to the current state of hypersonic aeroelastic and aerothermoelastic research. A more in depth review, is provided in a recent survey paper on panel flutter. ${ }^{32}$

The majority of panel flutter studies have focused on the supersonic regime ${ }^{72}$ (i.e. $2<M_{\infty}<4$ ) however, more recently hypersonic panel flutter has also been considered. ${ }^{41,73,74}$ The prevalent aerodynamic theory used in these studies is either piston theory, for $M_{\infty}>2.0$, or Van Dyke's second order theory for low to moderate supersonic Mach numbers $\left(1.2<M_{\infty}<2\right){ }^{32}$

Early studies, such as Refs. [17,75], dealt with relatively simple panel flutter analyses using first order quasi-steady aerodynamics (i.e. linear piston theory) and linear plate models. In particular, Ref. [17] used such a model to study the fundamental effects of aerodynamic heating on panel flutter. An early series of papers dealing with hypersonic aerothermoelasticity, ${ }^{25-27}$ identified panel flutter as significant for the design of re-entry vehicles, as well as hypersonic cruise vehicles. Furthermore, it was noted that panels are susceptible to a flutter instability when in-plane loading is present, suggesting that aerodynamic heating plays an important role in panel flutter. 
Early experimental studies of panel flutter revealed that the panels exhibited limit cycle oscillations rather than catastrophic failure, due to nonlinear membrane forces induced by moderate plate deflections. This limit cycle behavior was subsequently reproduced numerically for isotropic plates in Ref. [76] by implementing the moderate deflection von Karman plate theory. Subsequently, Ref. [77] extended this model to an orthotropic plate subject to arbitrary flow direction, where it was concluded that orthotropicity and flow direction have a strong effect on the flutter boundary of panels.

More recent studies of panel flutter have used finite elements to model relatively complex composite panels and temperature effects. Reference [78] included the effects of aerodynamic heating in isotropic plates using the finite element method for spatial discretization, while Refs. [79, 80] extended the model to orthotropic plates. The effect of panel location on the vehicle, panel curvature, and comparisons between piston theory and Euler aerodynamics in hypersonic flow was studied in Ref. [73]. The analysis was extended in Ref. [41] by introducing an improved formulation and solution process, increased generality in the plate temperature distributions, and a comparison of the unsteady pressure computed using piston theory, Euler and Computational Fluid Dynamic (CFD) Navier-Stokes solutions.

This comparison of the unsteady pressure ${ }^{41}$ is shown in Fig. 15. For $M_{\infty}=10.0$, and a typical plate vibration frequency, there was only a $5 \%$ difference between the unsteady pressure coefficient calculated using third-order piston theory and that calculated using the Euler equations. However, there was approximately a $60 \%$ difference between the Euler solution and the pressure coefficient calculated by solving the Navier-Stokes equations. This result emphasizes the important role viscosity may have in hypersonic aeroelasticity.

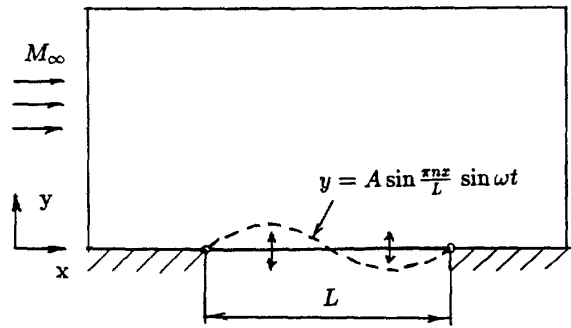

(a) Schematic of 2-D unsteady hypersonic flow field

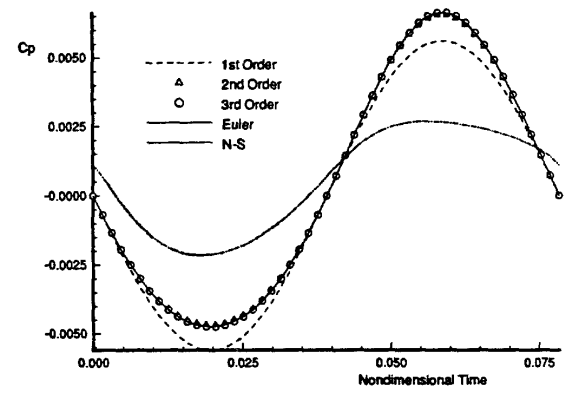

(b) Pressure Coefficient on the mid-panel

Figure 15. Comparison of unsteady pressures for an oscillating panel using different aerodynamic theories.[41]

Finally, it is evident from Refs. [81-85] that research into the advanced analysis of panel flutter is active and ongoing. In these studies, various in-plane edge restraints and imperfect geometry effects ${ }^{81}$ are investigated, as well as nonlinear aerothermoelastic panel flutter behavior ${ }^{82-84}$ and cylindrical panels under yawed high speed flow ${ }^{85}$

The general categories of panel flutter ${ }^{82}$ analysis are concisely listed in Table 4.

\section{Hypersonic Aeroelasticity of Wings and Complete Vehicles}

Because the complexity of the problem requires a much higher level of computational effort, when compared to the relatively convenient panel flutter problem, the aeroelastic behavior of wings and complete vehicles in hypersonic flow has not been studied as systematically and thoroughly as panel flutter. However, there are several experimental and computational studies that have examined fundamental issues of this problem.

Most of the experimental aeroelastic data generated in hypersonic flow was gathered in a series of studies conducted during the late 1950's and early 1960's using the Langley Hypersonic Aeroelastic wind tunnel. ${ }^{18-24,28,29}$ Conversely, most of the advanced computational work in hypersonic aeroelasticity has emerged relatively recently, beginning with the 1984-1994 period, and motivated primarily by the previously men- 
Table 4. Panel flutter analysis categories, as described in Ref. [82].

\begin{tabular}{cccc}
\hline \hline Type & Structural Theory & Aerodynamic Theory & Range of Mach No. \\
\hline 1 & Linear & Linear Piston & $\sqrt{2}<M_{\infty}<5$ \\
2 & Linear & Linearized potential flow & $1<M_{\infty}<5$ \\
3 & Nonlinear & Linear piston & $\sqrt{2}<M_{\infty}<5$ \\
4 & Nonlinear & Linearized potential flow & $1<M_{\infty}<5$ \\
5 & Nonlinear & Nonlinear piston & $M_{\infty}>5$ \\
6 & Nonlinear & Euler or Navier-Stokes equations & Transonic, supersonic, hypersonic \\
\hline \hline
\end{tabular}

tioned National Aerospace Plane Program (NASP). ${ }^{6,42,53,86-92}$ This section provides a review of the experimental and computational research conducted both experimentally and computationally on hypersonic vehicles and lifting surfaces.

\section{A. Experimental Aeroelastic Analysis of Hypersonic Configurations}

As interest increased in high speed flight during the 1950's, two early hypersonic experimental studies ${ }^{18,19}$ were conducted to examine the potential flutter of wing configurations proposed for high speed vehicles. Reference [18] performed experimental tests on the possible flutter of a dynamically and elastically scaled model of a proposed all-movable horizontal tail surface for the X-15. The prototype had an aspect ratio of 2.5, a taper ratio of 0.305 , and a sweep angle at the quarter-chord of $45^{\circ}$. No flutter was observed at the tunnel test conditions, at $M_{\infty}=6.86$. Analytical calculations of the flutter speed, using piston theory aerodynamics, indicated a flutter speed approximately four times higher than the velocity obtained in the tests. This is likely explained by the high stiffness of the model, where the first four free vibration frequencies were $44 \mathrm{~Hz}, 115 \mathrm{~Hz}, 148 \mathrm{~Hz}$, and $172 \mathrm{~Hz}$, respectively. In a similar study, ${ }^{19}$ the flutter of a low-aspect ratio, boost-glide-vehicle wing prototype was considered at $M_{\infty}=3.0$, and $M_{\infty}=7.3$. Similar to Ref. [18], none of the models tested experienced flutter at the tunnel operating conditions; again, primarily due to high model stiffness. The most flexible model had free vibration frequencies of $\omega_{1}=36.1 \mathrm{~Hz}, \omega_{2}=126.6 \mathrm{~Hz}, \omega_{3}$ $=244.1 \mathrm{~Hz}$, respectively

After these initial studies, more systematic, parametric investigations were undertaken by several researchers. In Ref. [21], the effect of planform geometry, airfoil thickness, and leading/trailing edge radii on the aeroelastic behavior of single- and double-wedge airfoils was studied experimentally for $0.7<M_{\infty}<6.86$. The tests in hypersonic flow were conducted on square planform, double-wedge airfoil, all-movable-controltype models; having leading and trailing edge radii from 0 to $6 \%$ chord, and airfoil thickness from 9 to $20 \%$ chord. For supersonic and hypersonic Mach numbers, analytical flutter results were computed using second-order piston theory. Hanson ${ }^{21}$ observed that increasing airfoil thickness had a destabilizing effect at the supersonic and hypersonic Mach numbers. Also, increasing airfoil bluntness had a stabilizing effect at the upper Mach number range. Finally, the agreement between the piston theory and experimental analysis was observed to improve with increasing Mach number. At $M_{\infty}=6.86$, the flutter boundary predicted using second-order piston theory aerodynamics was within $20 \%$ of the experimental flutter boundary. It also noted that the piston theory results were generally unconservative with respect to the experimental flutter boundaries.

In Ref. [22], an analytical and experimental flutter study of spring mounted cones in $M_{\infty}=6.83$ and $M_{\infty}=15.4$ flow conditions was conducted. Furthermore, analytical results were obtained using second-order piston theory, unsteady shock-expansion theory, and unsteady Newtonian aerodynamics. The cones tested had both pitch and plunge degrees of freedom, and a thickness-to-chord ratio of $26 \%$. The flutter speed index for this configuration as a function Mach number, and ratio of plunge to pitch natural frequency, are provided in Fig. 16. It is evident that unsteady Newtonian aerodynamics provide the best correlation with the experimental results, particularly for $M_{\infty}=15.4$. This is most likely due to the use of a relatively thick cone geometry, for which the use of Newtonian aerodynamics is most appropriate ${ }^{1}$

In Ref. [23], the effect of angle-of-attack and airfoil thickness on the binary flutter of a double-wedge airfoil section wing was investigated at $M_{\infty}=10.0$. The analytical results were generated using third-order 


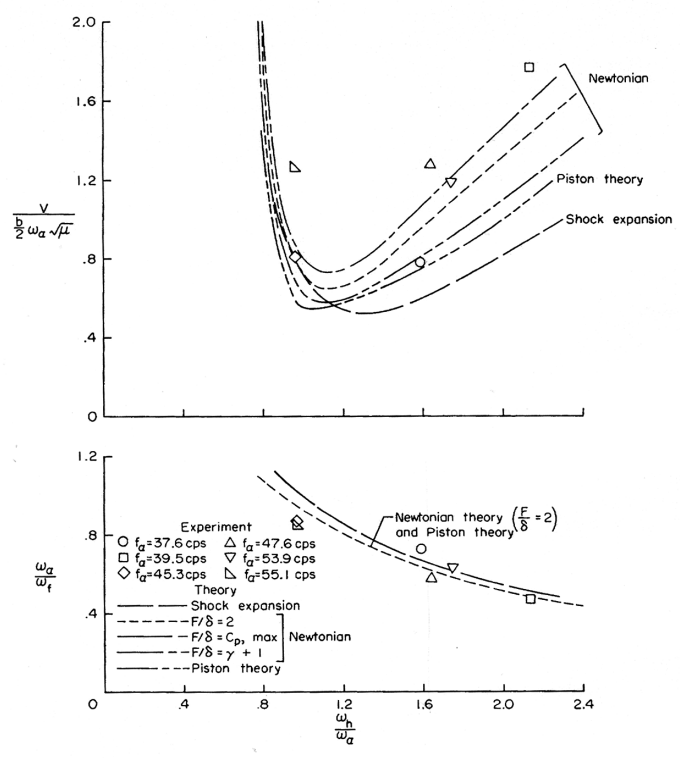

(a) Mach 6.83
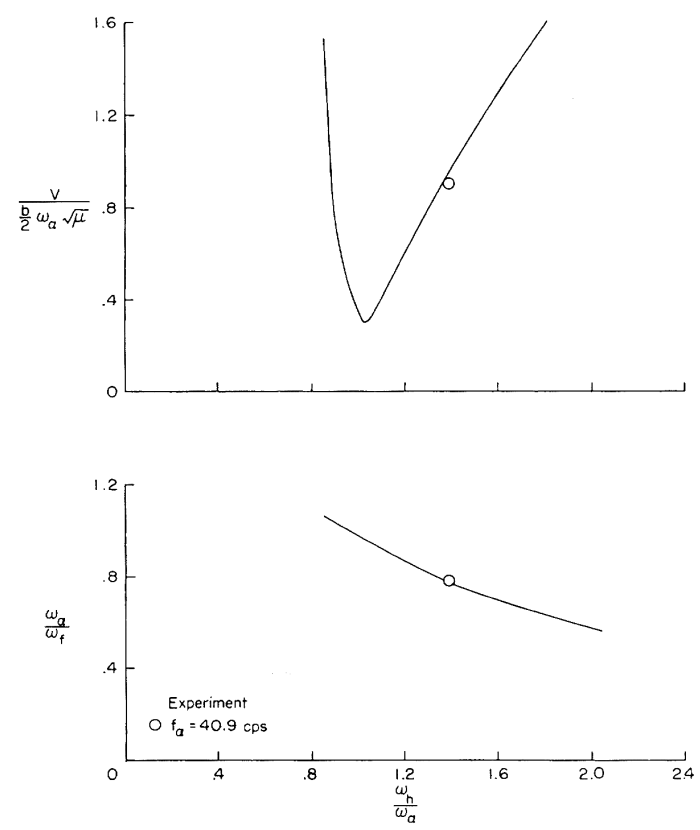

(b) Mach 15.4, Newtionan-Impact Aerodynamics

Figure 16. Experimental and analytical flutter boundary of a two-degree-of-freedom cone in hypersonic flow.[22]

piston theory aerodynamics. In particular, both an $11 \%$ and $15 \%$ thick airfoil section were tested at angles of attack ranging from $0^{\circ}$ to $10^{\circ}$. It was observed, similar to Ref. [21], that increasing the thickness, and angle-of-attack of the airfoils, decreased the flutter boundary. Also, the flutter velocity predicted using piston theory aerodynamics was generally within $6 \%$ of the experimental results.

In Ref. [24], the effect of leading edge bluntness on the hypersonic aeroelastic behavior of square planform, double-wedge airfoils was studied experimentally and analytically at $M_{\infty}=15.4$. Specifically, the leading and trailing edge radii were varied from $0,1,3$, and $6 \%$ of chord. Results indicated that increasing leading edge bluntness to $1 \%$ chord had a stabilizing effect, while additional increases in bluntess were destabilizing. Analytical results were generated using both unsteady Newtonian aerodynamics and also a combined Newtonian-piston theory method. In the combined method, Newtonian aerodynamics were used in the leading edge regions, while piston theory was used in the remaining sections. Differences, between the theoretical and analytical results, of up to $20 \%$ were observed in the flutter velocity. In general, however, the combined Newtonian-piston theory approach generated conservative results, while the Netwonian approach was unconservative.

In a follow-up study, ${ }^{28}$ an experimental and analytical investigation into the effect of leading-edge sweep was performed on the hypersonic aeroelastic behavior of delta-planform lifting surfaces. As before, the tests were conducted at $M_{\infty}=15.4$. Furthermore, several profile shapes were investigated, such as blunt single-wedges, double-wedges, and slabs; with leading-edge radii of $1.25 \%, 1.25 \%$, and $2.5 \%$ of their local chord, respectively. All of the profile shapes had a $5 \%$ maximum thickness-to-chord ratio. ${ }^{28}$ In general, the results indicated that increasing leading-edge sweep angle from $60^{\circ}$ to approximately $65^{\circ}$ or $70^{\circ}$ is destabilizing, and also that further increases were stabilizing. It was noted, however, that wind tunnel effects may have contributed to the higher sweep angle results. Goetz, ${ }^{28}$ also compared the results to those computed using piston theory and Newtonian unsteady aerodynamics. It was found that the piston-theory and Newtonian results were conservative, in terms of flutter velocity, with respect to the experimental results. Furthermore, the Newtonian results more closely predicted the experimental flutter velocities for all of the cases considered. The piston theory flutter velocities were up to $50 \%$ lower than the experimental flutter velocities, while the 
Newtonian flutter velocities were at most $25 \%$ lower than the experimental results.

In a third study, Goetz and Sewall ${ }^{29}$ performed an experimental investigation of a wing-fuselage configuration at $M_{\infty}=15.4$. In parallel, results were generated analytically using both piston theory aerodynamics, and a combination of piston theory and Newtonian aerodynamics. Results indicated that the analytical investigation provided similar trends, however, the flutter boundaries were unconservative by anywhere from $3 \%$ to $80 \%$. A possible explanation for the large discrepancies was the use of only the first four symmetric structural modes in the analysis. Thus, better correlation between the approximate and experimental results, due to higher-order modes, could not be ruled out. The model was destroyed during testing, however, before higher order modes could be measured. Note that the use of combined Newtonian-piston theory aerodynamics did not offer significant improvements in the flutter solution, compared to that obtained using only piston theory aerodynamics.

In a more recent study, ${ }^{42}$ the hypersonic aeroelastic behavior of several different delta-wing configurations, with both slab and double-wedge airfoil sections, was investigated experimentally in the NASA Langley Mach 20 Helium Tunnel. The configurations were tested at $16.7<M<18.1$, and reduced frequencies below 0.09. Furthermore, the results were compared to analytical predictions that were computed using linearized second-order piston theory aerodynamics. Note that both blunt and sharp leading/trailing edges were considered, and that the double-wedge airfoils had a $4 \%$ thickness-to-chord ratio. A number of conclusions were made, namely: 1) the slab airfoils were more stable than the double-wedge airfoils, 2) the blunt leading edges improved stability compared to the sharp leading edges (leading edge radii not specified), and 3) the experimentally measured flutter dynamic pressures were substantially lower than the analytical results. Further analysis in Ref. [42] deomonstrated that modifying the airfoil shapes with a laminar flat plate boundary layer displacement thickness, as given in Eqn. 38, improved the agreement between the analytical and experimental results. This is shown in Fig. 17. This result emphasizes the potential impact of viscous effects in the hypersonic regime on the aeroelastic behavior of hypersonic vehicles.

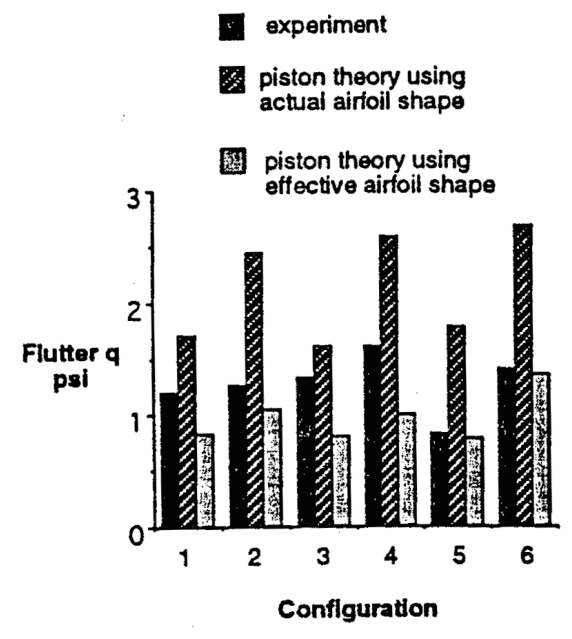

Figure 17. Comparison of analytical and experimental dynamic pressures at flutter.[42]

\section{B. Computational Aeroelastic Analysis of Hypersonic Configurations}

Advanced CAE analysis, where CFD flow analysis and finite element structural analysis (FEA) is integrated into the full aeroelastic solution procedure, is currently the state-of-the-art method for the aeroelastic study of hypersonic vehicles. However, as will be seen from a review of the research in this area, significant work is still necessary in order to reach the full potential of advanced computational approaches.

The NASP program (1984 to 1994) represents the initial phases of using advanced CAE analysis to design hypersonic vehicles. Much of the aeroelastic research for the NASP was summarized during two special sessions held at the 34th AIAA/ASME/ASCE/AHS/ACS Structures, Structural Dynamics, and Materials Conference in 1993. Note that only the studies pertaining to hypersonic flow are reviewed here. 
The research presented at these sessions focused on the X-30 NASP Demonstrator Model, an unclassified version of the National Program Office (NPO) classified finite element model. An overview of the research effort is presented in Ref. [6].

In Ref. [87], a supersonic and hypersonic aeroelastic analysis of the NASP Demonstrator Model was performed using second order Van Dyke quasi-steady aerodynamics for $1.6<M<8.0$; corresponding to reduced frequencies of $0.04<k_{\omega}<0.18$. As shown in Fig. 18a), it was predicted that body-freedom flutter was present, at all Mach numbers analyzed, due to coupling of the short-period mode with the wing-pivot mode. A weaker elastic mode flutter involving the coupling of the wing-pivot mode with the fuselage-bending mode was also identified at $M=2.3$. It was also observed that shifting the all-moveable wing aft on the fuselage is destabilizing. This result is illustrated in Fig. 18b). It was noted that in all the cases considered, flutter involved the all-movable wing-pivot vibration mode.

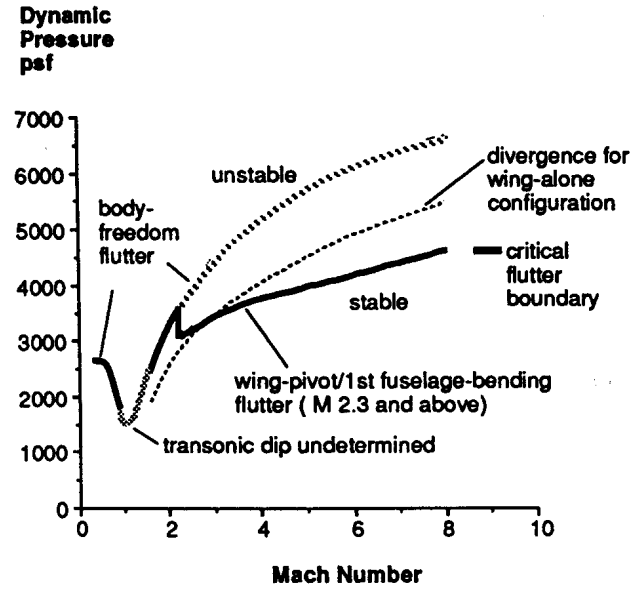

(a) Predicted stability boundaries for the NASP demonstrator

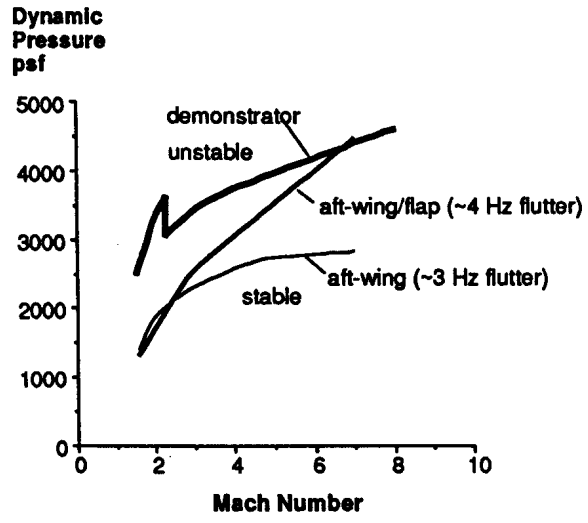

(b) Comparison between the stability boundaries for the baseline demonstrator design, and two additional variations

Figure 18. Critical supersonic/hypersonic flutter boundaries for a NASP demonstrator model.[87]

Noting that hypersonic flows are often quasi-steady in nature due to relatively low values of reduced frequency $\left(k_{\omega} \ll 1\right)$, an improved method for calculating quasi-steady generalized forces using steady CFD calculations was developed in Ref. [89]. Separate solutions for the real and imaginary portions of the pressure were obtained using carefully constructed boundary conditions reflecting the unsteadiness of the flow. To investigate the method, the authors studied a wing operating at Mach numbers of 5, 10, and 15, and reduced frequencies of 0.05 and 0.1 . It was observed, by comparing results with complete unsteady CFD calculations of the hypersonic configuration, that the CFD-based quasi-steady approach offered an improvement in accuracy over linear piston theory. ${ }^{89}$

In addition to these aeroelastic studies of the NASP vehicle effort, there have been several papers on different and newer hypersonic vehicle configurations such as the X-33, X-34 and X-43. The X-33 was a 1/2-scale fully functional technology demonstrator for the Lockheed-Martin VentureStar, while the X-34 was developed by the Orbital Sciences Corporation for NASA's RLV program. The X-43 was used in the NASA Hyper-X Project, mentioned earlier, to demonstrate the feasibility of air-breathing SCRamjet engine technologies. Schematic depictions of these vehicles are shown in Fig. 1.

In Ref. [93], the aeroelastic behavior of the X-34 launch vehicle in free flight was studying using MSC NASTRAN. This code is primarily a finite element structural/heat transfer code; however, there is an aeroelastic module available that utilizes approximate aerodynamics. For the hypersonic regime, MSC NASTRAN uses piston theory aerodynamics. The aeroelastic behavior of the vehicle was studied along a representative trajectory, with a maximum Mach number of $M=8.0$. The study found instabilities at speeds above $M=5.0$, in the primary bending modes, due to the control system.

In Ref. [7], a CFD-based flutter analysis was used for the aeroelastic analysis of the X-43 configuration, 
using system identification based order reduction of the aerodynamic degrees of freedom. The system identification was carried out using an Auto-Regressive Moving Average (ARMA) model; which describes the modal response force of a system at a given time as a summation of scaled previous outputs, and scaled values of modal displacement inputs to the system, for small perturbations of the system. Both the structure and the fluid were discretized using the finite element approach. The results of the analysis are shown in Fig. 19, where it is evident that the flutter point is predicted similarly for $M_{\infty}=7.0$, using either Euler or piston theory aerodynamics. The order of the piston theory model, however, was not specified.

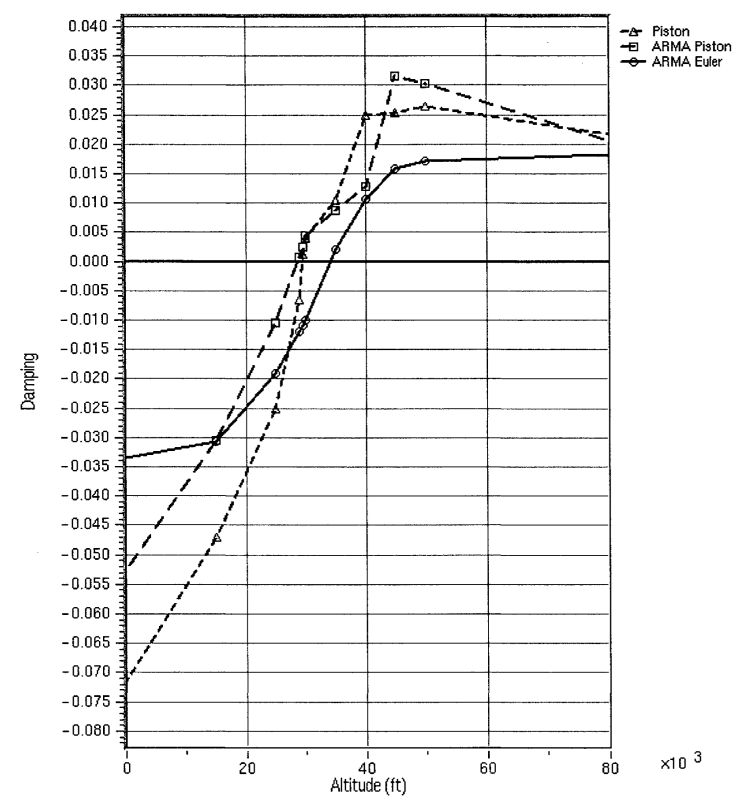

Figure 19. Flutter boundary of the X-43 hypesonic vehicle, at Mach 7.0, predicted using piston theory, ARMA piston theory, and ARMA Euler unsteady aerodynamics.[7]

Recently, the authors of this paper have been co-investigators in a number of studies on the hypersonic aeroelastic behavior of generic reusable launch vehicles. ${ }^{33,44-50}$ The aeroelastic analysis was performed using computational tools such as the NASA Langley CFL3D ${ }^{71}$ and an independently generated aeroelastic code based on piston theory aerodynamics. The CFL3D code contains both a CFD solver, and a deforming mesh capability enabling aeroelastic computations. ${ }^{71}$ The method of solution is shown in Fig. 20, and is briefly described next.

The first step in Fig. 20 is generation of the vehicle geometry using a CAD software package. Using this geometry, a mesh generator is used to create a structured mesh for the flow domain around the body. In parallel, an unstructured mesh is generated, representing the surface of the finite element model of the vehicle. This structural model is further refined with internal stiffeners and mass elements to represent a complete vehicle, and is used to obtain the free vibration modes of the vehicle using MSC NASTRAN. Subsequently, the fluid mesh is used to compute the flow around the rigid vehicle using the CFL3D code CFD solver. ${ }^{71}$ In order to generate the aeroelastic input for CFL3D, the modal deformation at each surface gridpoint in the fluid mesh is obtained by using cubic interpolation (in MATLAB) from the finite element structural model, for each structural mode. Using the flow solution as an initial condition, and the interpolated modal data as additional input, an aeroelastic equilibrium state is obtained for the flexible vehicle. For a geometry with vertical symmetry at zero angle-of-attack, the equilibrium state is the same as the undeflected state. Next, the structure is perturbed in one or more of its modes by an initial modal velocity condition, and the transient response of the structure is obtained. To determine the flutter conditions at a given altitude, aeroelastic transients are computed at several Mach numbers and the corresponding dynamic pressures. The frequency and damping characteristics of the transient response for a given flight condition and vehicle configuration 
are then determined using a time domain damping and frequency identification method, such as the ARMA curve fitting method. ${ }^{49,94-96}$

The approach presented in Fig. 20 is applied at the same altitude and vehicle configuration for a range of Mach numbers, resulting in a series of damping values for the system. The flutter Mach number can then be estimated from this series by interpolating the damping data points to identify the value of the Mach number at which the damping is zero.

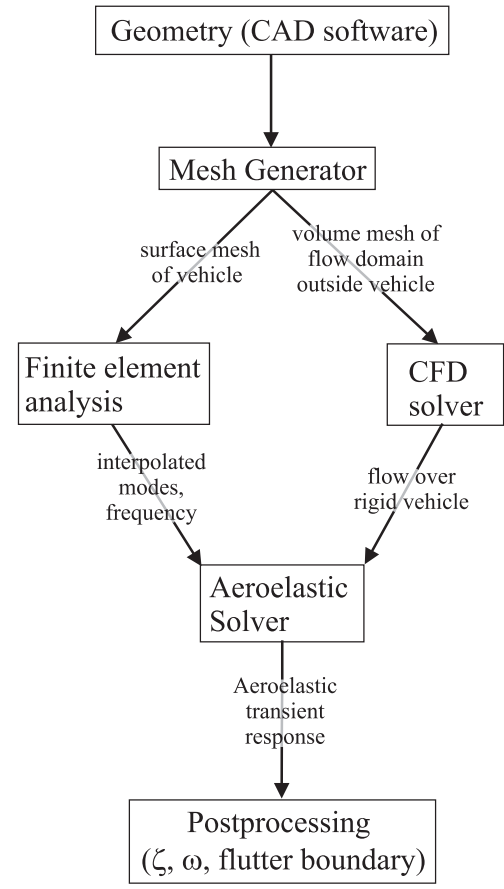

Figure 20. A flow diagram of the computational aeroelastic solution procedure.[33, 44-50]

In Ref. [33], using both Euler and Navier-Stokes aerodynamics, the above approach was used to compute the aeroelastic behavior of the double-wedge airfoil shown in Fig. 21. The results were then compared to those generated using several approximate aerodynamic theories, in order to characterize the range of validity of the approximate methods, and also investigate further the importance of viscosity in the hypersonic regime. The theories were compared over a wide range of operating conditions by varying the elastic axis location, and operating altitudes. The approximate aerodynamics were computed using piston theory (PT), Van Dyke's second order theory (VD), unsteady shock-expansion theory (SE), and unsteady Newtonian-impact theory (NI). An important part of the investigation ${ }^{33}$ was the generalization of the results in terms of the hypersonic similarity parameter. ${ }^{1}$ This parameter is useful since it describes similarity for different flow/body combinations. In other words, moderate Mach number flow around a thick body is similar to high Mach number flow around a thin body, if the two systems have the same hypersonic similarity value. Note that hypersonic similarity is governed by $M_{\infty} \theta_{l}$. For the double-wedge airfoil shown in Fig. 21, this is equivalent to $M_{\infty} \tau$.

In the first part of the analysis, ${ }^{33}$ comparisons were made between first, second, and third order piston theory. This is shown in Fig. 22. It was concluded from these results that second and third order effects are important for high Mach number flows. At each of the operating altitudes, the first order piston theory boundaries were unconservative compared to both the second and third order piston theory boundaries. More specifically, first order piston theory results were $20 \%$ higher than the second and third order piston theory results for $M_{\infty} \tau=0.18\left(M_{\infty}=5.0\right)$. Furthermore, the differences increased with increasing Mach number. This result emphasizes the destabilizing effect of thickness in hypersonic flows, since it is neglected using first order piston theory.

In the second part of the analysis, ${ }^{33}$ CFD-based results were generated and compared to several approximate theories. These results are provided in Fig. 23. Since there is less than a $15 \%$ difference in the results for $M_{\infty} \tau<1.0\left(M_{\infty}<30\right)$, it is evident that the third order PT, VD, and SE results match the CFD-based 


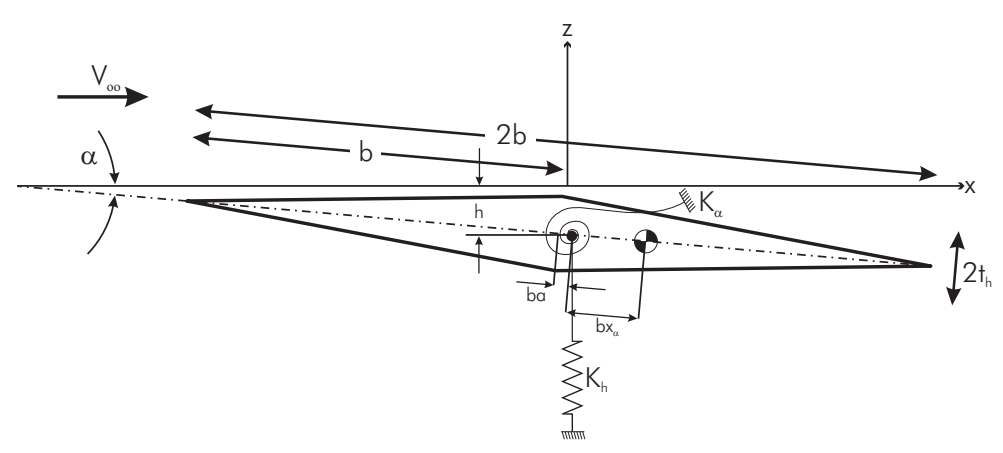

Figure 21. Two degree-of-freedom typical section geometry.[33]

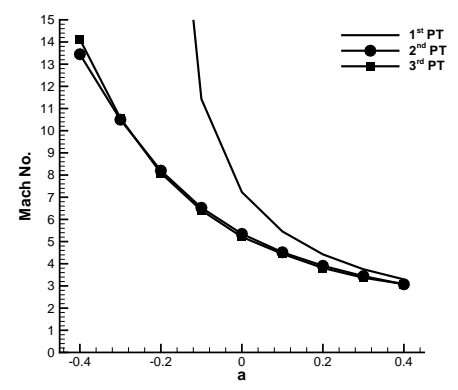

(a) 10,000 feet

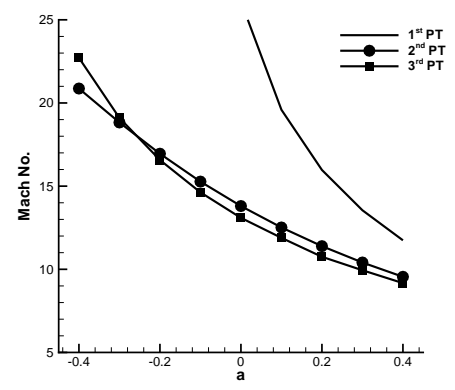

(b) 40,000 feet

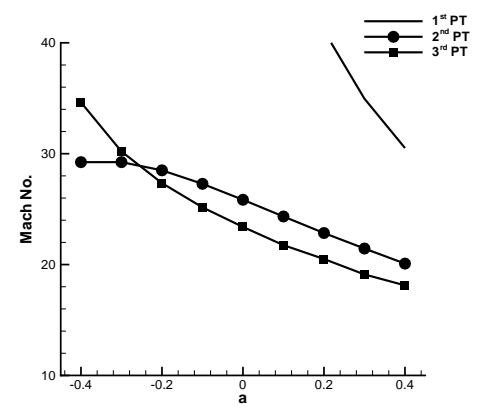

(c) 60,000 feet

Figure 22. Variation in the flutter Mach number of a double-wedge typical section, as a function of elastic axis offset parameter $a$, computed using different orders of piston theory aerodynamics. [33]

results reasonably well, for moderate to high Mach numbers. The NI impact results, however, were unconservative for most of the cases considered. This is likely due to the relatively thin geometry considered in the study. ${ }^{33}$ Finally, the inclusion of viscosity did not significantly affect the aeroelastic behavior of the airfoil.

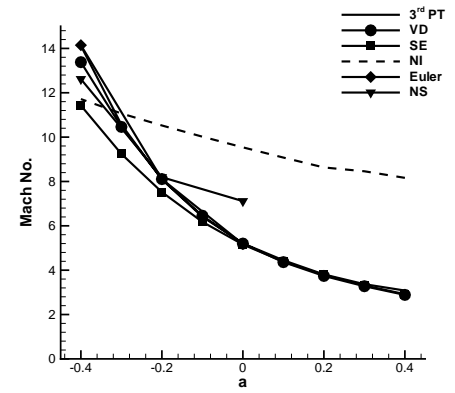

(a) 10,000 feet

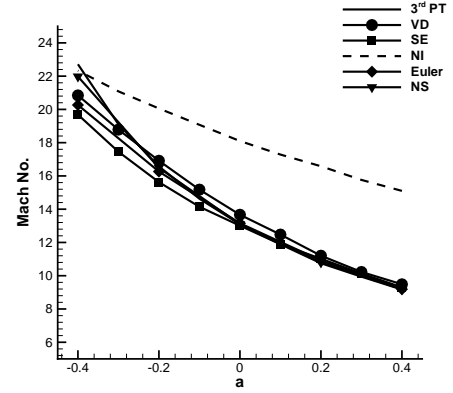

(b) 40,000 feet

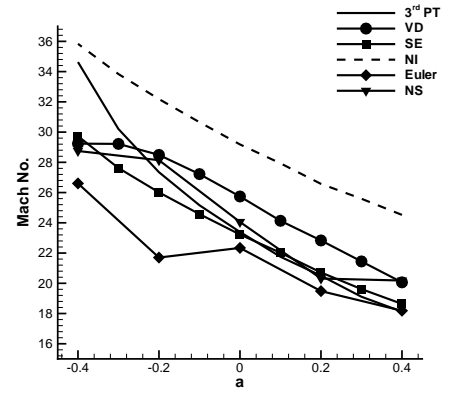

(c) 60,000 feet

Figure 23. Variation in the flutter Mach number of a double-wedge typical section, as a function of elastic axis offset parameter $a$, computed using several different aerodynamic models.[33] 
In the final part of the analysis, ${ }^{33}$ the aeroelastic behavior of the airfoil was computed using piston theory with an effective shape added to the airfoil surface. The results were compared to those generated by solving the unsteady Navier-Stokes equations. The effective shapes were computed using Eqn. 38, for a flat plate correction ("FP"), and Eqn. 45 for the CFD correction ("CFD"). Figure 24 provides a comparison of the two effective shapes for a typical flutter Mach number of the airfoil at 60,000 feet. It is clear that the flat plate correction significantly underpredicted the thickness of boundary layer over the aft portion of the airfoil. ${ }^{33}$ The aeroelastic behavior of the airfoil, computed using the effective shape corrections, are shown in Fig. 25. It was observed that the addition of an effective shape did improve the correlation, between the approximate aerodynamic and the full CFD-based analysis, at high Mach numbers.

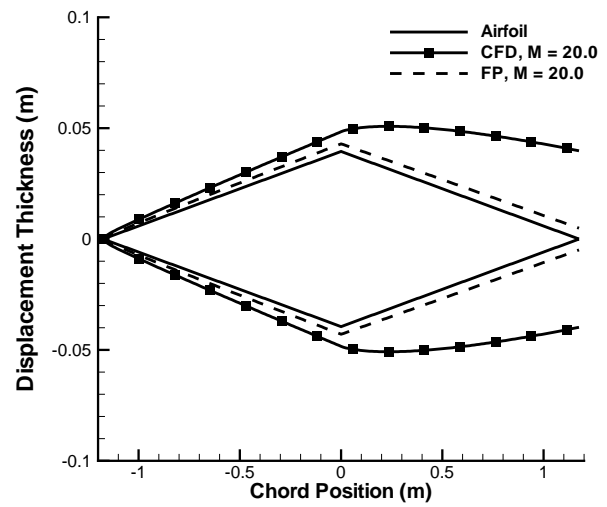

Figure 24. Comparison of effective shapes generated using both a laminar flat plate (FP) approximation and steady CFD pressure data, at $\mathbf{6 0 , 0 0 0} \mathrm{ft}, \mathbf{R e}=1.8 \times 10^{8} \cdot[\mathbf{3 3}]$

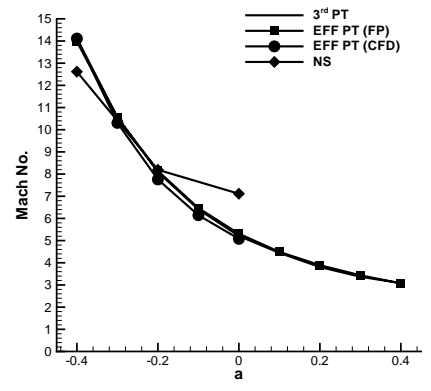

(a) 10,000 feet

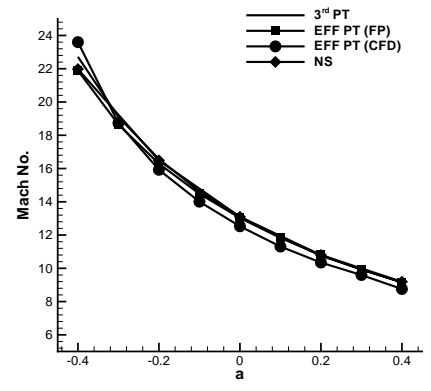

(b) 40,000 feet

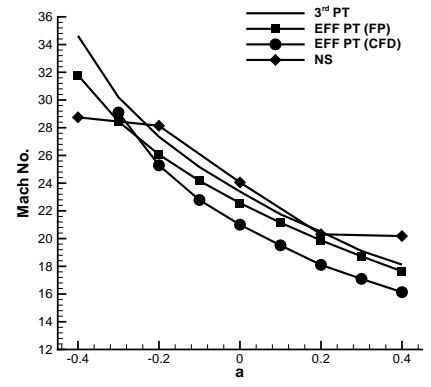

(c) 60,000 feet

Figure 25. Variation in the flutter Mach number of a double-wedge typical section, as a function of elastic axis offset parameter $a$, computed using third order piston theory, third order piston theory with effective shape corrections, and Navier-Stokes aerodynamics.[33]

The CAE method of solution in Fig. 20 was also applied to a generic hypersonic vehicle configuration ${ }^{44-50}$ resembling the X-33 RLV, as shown in Fig. 26, and the three-dimensional low-aspect ratio wing ${ }^{33,47,49}$ shown in Fig. 8. The low-aspect ratio wing was considered representative of a fin or control surface on a hypersonic vehicle. The flutter boundaries for these two configurations are shown in Figs. 27 and 28. A number of important conclusions were drawn from these results.

For both of the configurations investigated, the flutter boundary was not sensitive to grid resolution at moderate hypersonic Mach numbers. At the upper Mach number ranges, however, grid resolution was important since the shock layer approached the body surface, and required increased grid density to re- 
solve. ${ }^{33,50}$ Also, grid resolution was important for the Navier-Stokes results at the very low altitudes, in order to properly capture the boundary layer. ${ }^{33}$ However, it is not expected that hypersonic vehicles would fly at hypersonic Mach numbers at such low altitudes.

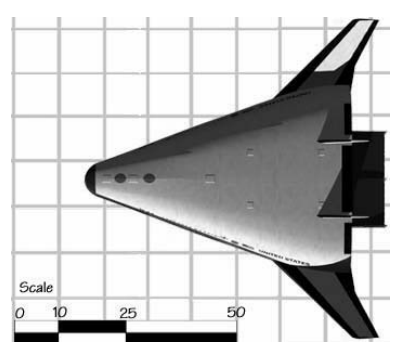

(a) X33, top view.

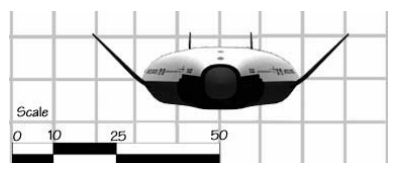

(c) X33, front view.

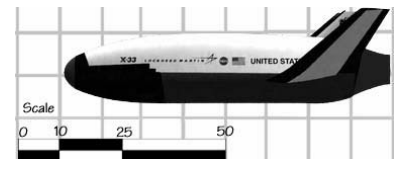

(e) X33, side view.

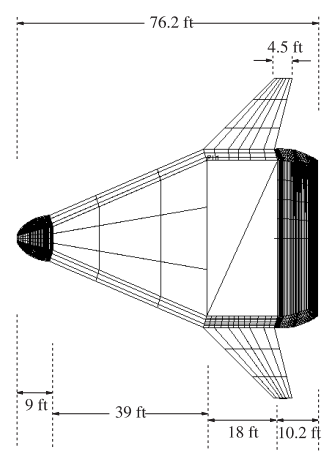

(b) Generic vehicle, top view.

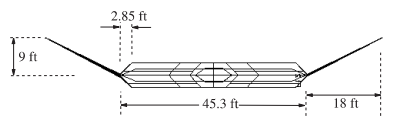

(d) Generic vehicle, front view.

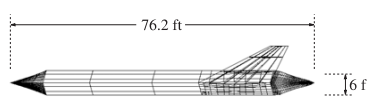

(f) Generic vehicle, side view.

Figure 26. X-33 and generic reusable launch vehicle.[44-50]

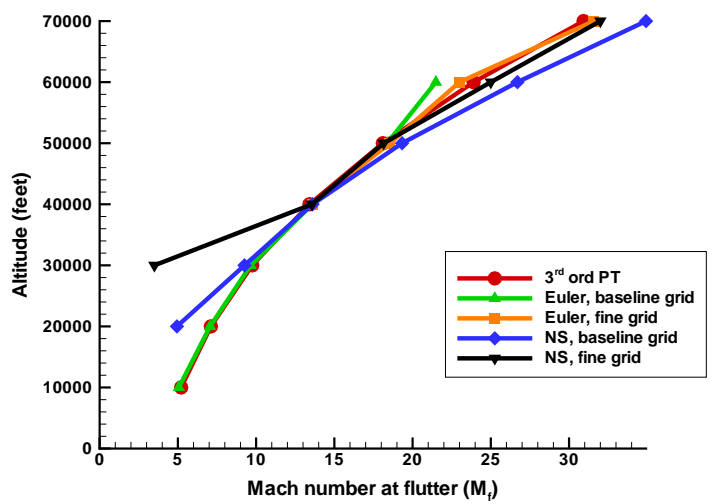

(a) Mach number

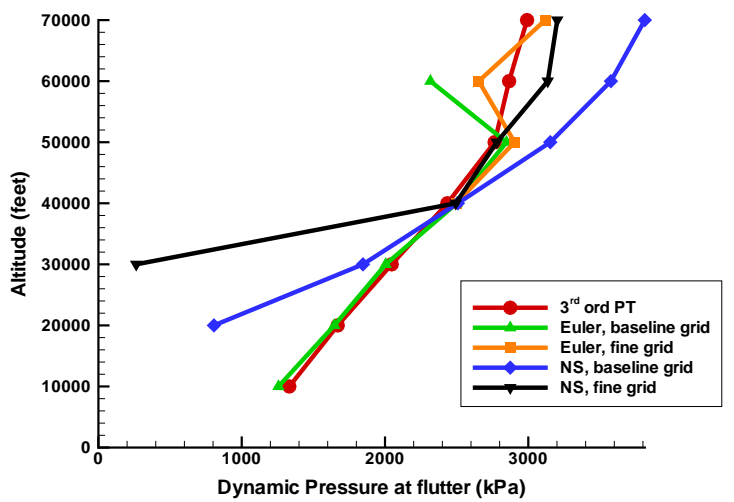

(b) Dynamic Pressure

Figure 27. Flutter envelope of the low-aspect ratio wing, calculated using third order piston theory, Euler and Navier-Stokes aerodynamics.[33]

Another important observation, from the results presented in Figs. 27 and 28, is that the differences between the Euler and third order piston theory flutter Mach numbers for the wing was only 5-8\%, while 


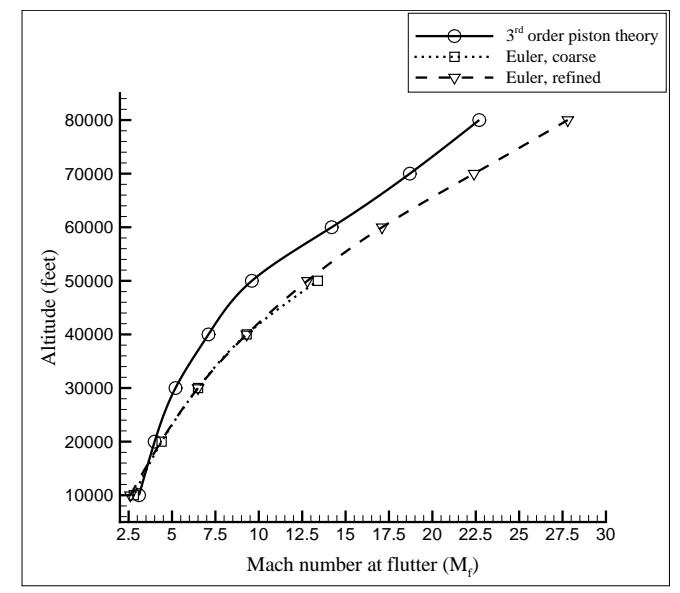

(a) Mach number

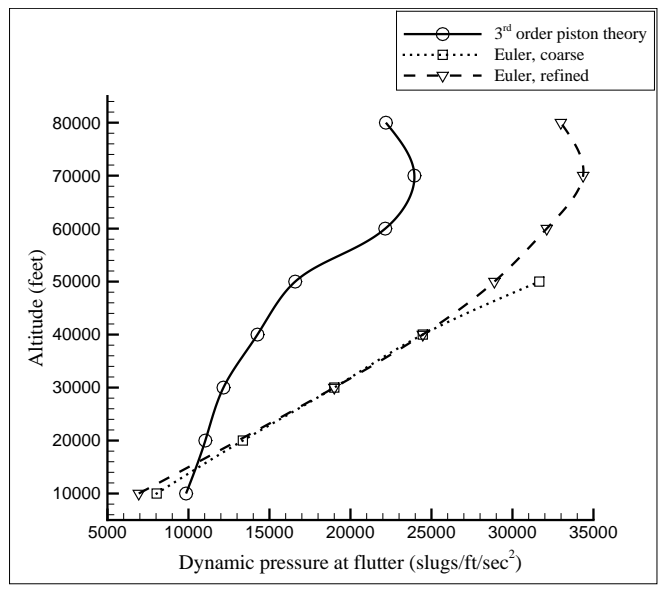

(b) Dynamic Pressure

Figure 28. Flutter envelope of the generic hypersonic vehicle, calculated using piston theory and Euler aerodynamics. $[49,50]$

the differences grew to over $25 \%$ when the complete vehicle was considered. It was concluded that this was due to increased three-dimensional flow effects, on the complete vehicle, introduced by flow interaction between the lifting body and the canted fins. Such effects are not properly captured by the sectional piston theory aerodynamics. ${ }^{50}$ It was also observed that, due to the hypersonic speed of the flow, the configurations experienced flutter at low reduced frequencies $\left(\left(k_{\omega}\right)_{\text {wing }}<0.5,\left(k_{\omega}\right)_{\text {vehicle }}<0.3\right)$. Thus, third-order piston theory, a quasi-steady aerodynamic theory, is a valid approach to modeling the unsteady aerodynamics so long as the body is thin and there are minimal three-dimensional flow effects. ${ }^{33,50}$

It is evident from results computed using both inviscid and viscous flows, i.e. Figs. 15, 17, 23, 25, and 27 , that the effect of viscosity in hypersonic aeroelasticity is dependent on the operating conditions. For some of the configurations and operating conditions, there were significant differences between the two types of flows. Conversely, in others, there were not. Thus, more work is needed to better understand the role viscosity plays on this class of vehicles.

In a related note, it is also clear that certain aeroelastic systems will require the use of more sophisticated aerodynamics than the commonly used approximate theories. Namely, configurations where threedimensional flow and viscous effects are important. Finally, it is apparent from the various flutter boundaries presented, e.g. Figs. 19, 23, 27, and 28, that the neglect of aerodynamic heating in the aeroelastic analysis results in high flutter Mach numbers at relatively low altitudes. Thus, since aerodynamic heating is extreme at such operating conditions, this effect must be incorporated in order to compute the true aeroelastic behavior.

\section{Hypersonic Aerothermoelasticity}

Aerodynamic heating of a hypersonic vehicle degrades structural material properties; and also introduces thermal stresses in the airframe ${ }^{97-99}$ due to rapidly changing conditions of heat input where time lags are involved, or from equilibrium conditions of non-uniform temperature distribution. ${ }^{98,99}$ Lowered stiffness, due to material degradation and thermal stresses, results in a reduction in natural frequencies of the structure. ${ }^{98-100}$ Aerodynamic heating of the flow surrounding a hypersonic vehicle leads to significantly different thermodynamic and transport properties, high heat-transfer rates, variable $\gamma$, possible ionization, and nonadiabatic effects from radiation. ${ }^{97,101}$ Each of these issues can significantly affect the aeroelastic behavior of a hypersonic vehicle.

The heat transfer between the fluid and the structure, schematically depicted in Fig. 29, is determined 
from an energy balance of the heat fluxes at the wall of the structure: ${ }^{10,102}$

$$
\dot{q}_{a e r o}=\dot{q}_{r a d}+\dot{q}_{c o n d}+\dot{q}_{s t r d}
$$

where

$$
\begin{gathered}
\dot{q}_{a e r o}=h_{h t}\left(T_{A W}-T_{W}\right) \\
\dot{q}_{r a d}=\sigma \epsilon T_{W}^{4} \\
\dot{q}_{c o n d}=\left(\kappa \frac{\partial T}{\partial \mathbf{n}}\right)_{W} \\
\dot{q}_{s t r d}=\rho_{W} c_{p w} \Delta_{w} \frac{d T_{W}}{d t}
\end{gathered}
$$

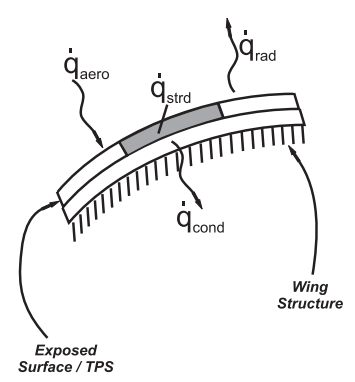

Figure 29. Heat transfer at the wall of a hypersonic vehicle.[49]

Thus, the heat transfer represents a balance between the convective heating by the fluid $\left(\dot{q}_{\text {aero }}\right)$ and heat loss due to conduction into the structure $\left(\dot{q}_{c o n d}\right)$, radiation out to space $\left(\dot{q}_{\text {rad }}\right)$, and energy stored in the wall $\left(\dot{q}_{s t r d}\right)$. The heat transfer problem is driven by Eqn. 47 , or more specifically, the adiabatic wall temperature, $T_{A W}$, which is a function of the surface geometry and free stream conditions.

Several early publications, such as Refs. [17,25,97,98], provide insight into the salient aspects of aerothermoelasticity. In Refs. [17,98], aerothermoelasticity is described as the expansion of Collar's aeroelastic triangle to include the coupling of aerodynamic heating. This is graphically depicted by the lower portion of the Aero-servo-thermo-elastic hexahedron shown in Fig. 3. Figure 30 illustrates the degree of coupling between the aerodynamic heating, aerodynamic forces, inertial forces, and elastic forces. ${ }^{98}$

Often, the aerothermoelastic problem can be simplified by neglecting the "weak" coupling shown in Fig. 30, as well as the effect of the aerodynamic pressure on the aerodynamic heating. This reduces the analysis to an aerothermal problem and a separate aeroelastic problem. Figure 31 illustrates how this is implemented through finite element analysis. ${ }^{103}$ In this solution procedure, the aerothermal solution is obtained first, and the aeroelastic analysis is then carried out on the updated structure. This simplification of the aerothermoelastic problem relies on three important assumptions: ${ }^{17,25,97,98}$ 1) thermodynamic coupling between heat generation and elastic deformation is negligible; 2) dynamic aeroelastic coupling is small, i.e. the characteristic time of the aerothermal system is large relative to the time periods of the natural modes of the aeroelastic system; and 3) static aeroelastic coupling is small, i.e. total elastic deflections are insufficient to alter the temperature distribution. In most cases, the first two assumptions are valid, however, it is possible for aerodynamic pressures to cause deformations of the wing such that the local angle-of-attack, and therefore the heat distribution in the wing, is altered. ${ }^{17}$

The amount of published research in the field of hypersonic aerothermoelasticity is limited. Furthermore, due to the complexity of the problem, all of the studies conducted have implemented varying degrees of approximation. In an early aerothermoelastic study ${ }^{104}$ the effect of thermal stresses on the static aeroelastic stability of a cantilever wing with a double-wedge cross-section was examined. Thermal stresses were introduced into the wing using a chord wise parabolic heat distribution. It was determined that thermal stresses affected the stability of the wing by influencing both torsional rigidity and the anticlastic effect.

The effect of aerodynamic heating on divergence, control effectiveness, and flutter was considered in Ref. 


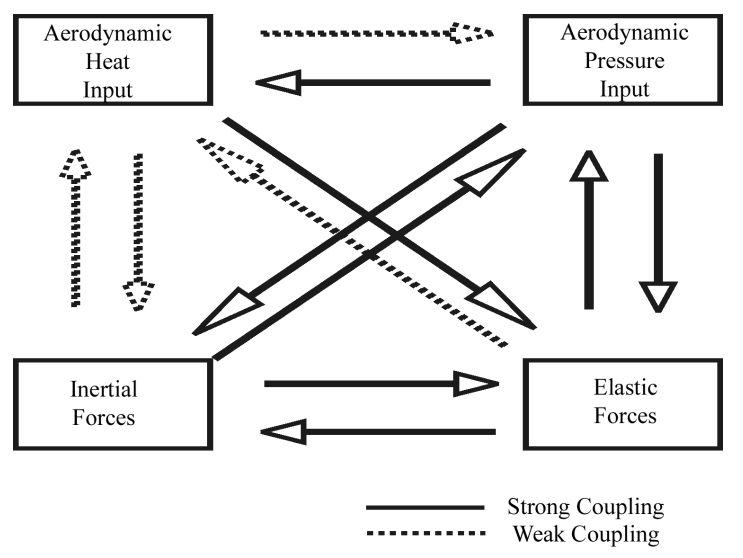

Figure 30. Degree of coupling for the domain of aerothermoelasticity. [98]

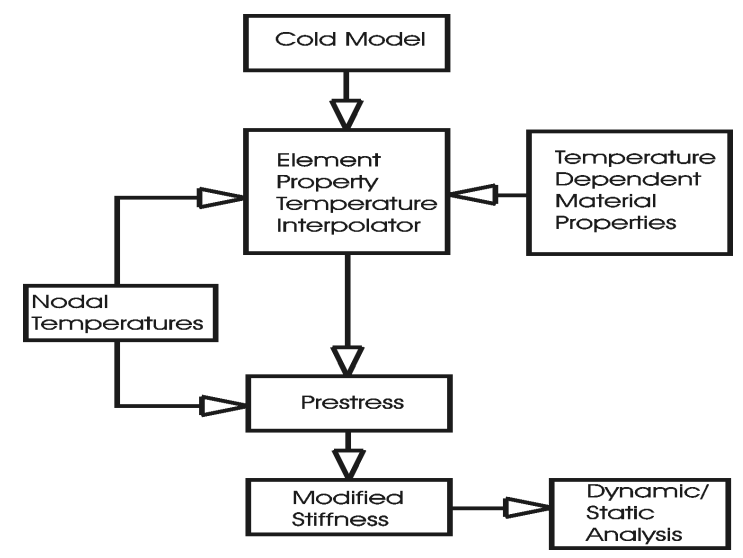

Figure 31. Proposed process for including thermal effects into the finite element analysis of a aerodynamically heated structure.[103]

[17]. In this study, the flutter boundary of a solid aluminum wing, with a rectangular planform (6 ft semispan) and $4 \%$ thick double-wedge airfoil section ( $3 \mathrm{ft}$ chord), at $35,000 \mathrm{ft}$, was reduced by up to $60 \%$ due to material property degradations in the presence of small temperature gradients.

An aerothermoelastic analysis of a thin wing in heated flow was carried out in Ref. [105], in order to determine the effect of thermal stresses on aeroelastic stability, and to develop approximate methods for predicting this behavior. In this study, a flutter analysis of a solid wing, with a rectangular planform and $65 \mathrm{~A}$ series airfoil, was performed using both an experimental and analytical approach. The wing was placed in Mach 2 flow preheated to $300^{\circ} \mathrm{F}$ and $800^{\circ} \mathrm{F}$. It was found that the wind tunnel model did not flutter for the lower temperature flow $\left(300^{\circ} \mathrm{F}\right)$. However, when the flow was heated to $800^{\circ} \mathrm{F}$, the model began to flutter after $2 \mathrm{sec}$, and continued to flutter for 2 additional seconds, at which point aeroelastic stability was gradually regained. The analytical results, based on Van Dyke's second order supersonic theory for the unsteady aerodynamics, were capable of adequately reproducing this result. Results from Ref. [100] were used to determine the reduction in stiffness due to thermal stresses.

The aerothermoelastic characteristics of an aluminum finned missile in the Mach number range $3 \leq$ $M \leq 6$ were studied in Ref. [106]. The analysis considered both a constant temperature and a parabolic temperature distribution in the chord wise direction, and neglected span wise distributions. The maximum temperature considered was $650^{\circ} \mathrm{F}$. The unsteady aerodynamic loads were computed using third order piston theory aerodynamics. The authors chose not to perform comparisons between the heated and unheated configuration, but rather studied the effect of temperature distribution on the flutter boundary. It was determined that the parabolic temperature distribution resulted in a lower flutter speed when compared to the isothermal distribution.

A limited number of aerothermoelastic studies have also been performed on the NASP vehicle project discussed earlier. An aerothermoelastic analysis of a NASP vertical fin, using several different temperature distributions, was performed in Ref. [86]. The aerodynamic heating was approximated using experimental data generated from flights of the NASA X-15 experimental aircraft, scaled to reasonable values for the aluminum model used in the analysis. Several different temperature were used, as illustrated in Fig. 32, ranging from $600^{\circ} \mathrm{F}$ at the leading edge, to $100^{\circ} \mathrm{F}$ at the trailing edge of the fin. A finite element model was used to determine the structural characteristics using the different temperature distributions. It was determined that two modes were of primary importance; namely the "flap mode", which consisted of control surface rotation about a hinge line, and a fin bending mode, which consisted of a primary bending mode with some control surface rotation. The "flap mode" natural frequency was reduced by nearly $50 \%$, while the bending mode frequency was reduced by approximately $20 \%$. The unsteady aerodynamic loads were computed using Van Dyke's second order theory. As shown in Fig. 33, the relatively constant temperature distributions had little effect on the flutter boundary. However, the "spike 1" distribution decreasead the flutter boundary by up to $37 \%$. This emphasizes the importance of chord-wise thermal gradients, and thermal stresses, on the flutter behavior of hypersonic vehicles.

In Ref. [53], an aerothermoelastic analysis of the NASP vehicle was conducted using a blended Van Dyke/Newtonian impact theory for the calculation of the aerodynamic loads. The effect of aerodynamic 


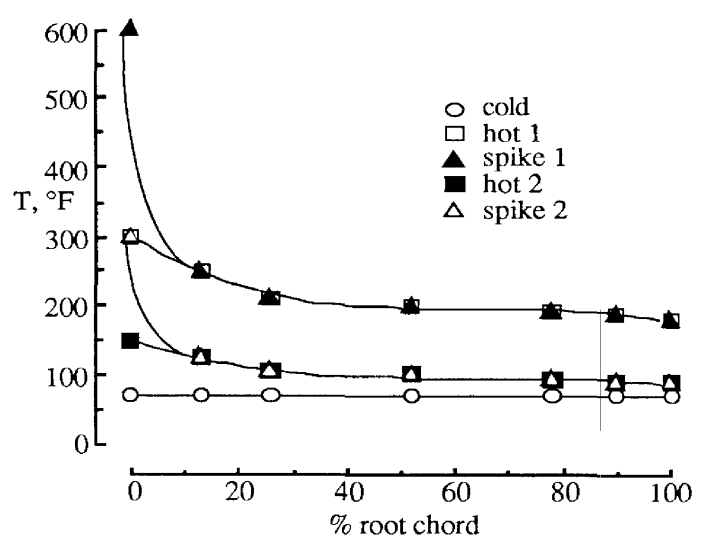

Figure 32. Temperature distributions applied to a NASP type vertical fin.[86]
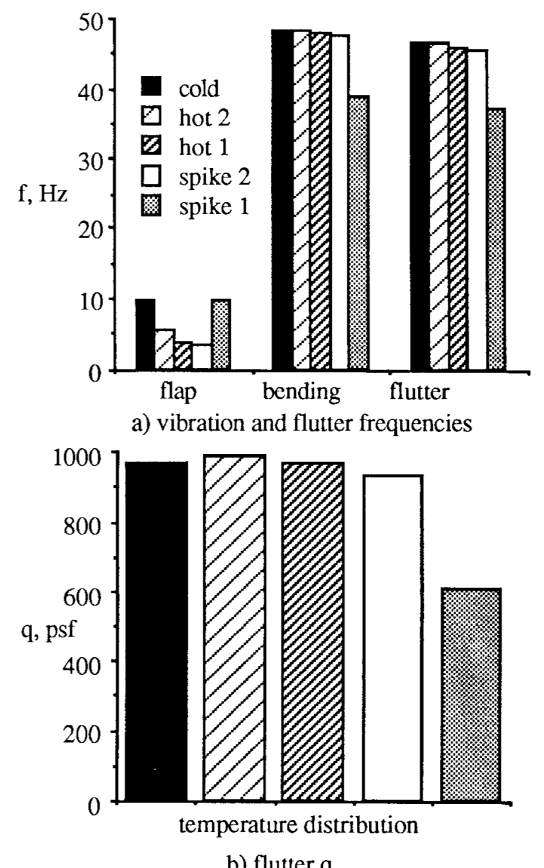

b) flutter $q$

Figure 33. Effects of aerodynamic heating on the frequencies and flutter boundary of a NASP type vertical fin. $[86]$

heating on the aeroelastic behavior of the vehicle was approximated by modifying the material properties of the structure due to increased temperature. The structural analysis was performed using the X-30 NASP Demonstrator finite element model; and surface temperatures on the vehicle were obtained with the Aerodynamic Preliminary Analysis System code (APAS). ${ }^{107}$ It was noted that the highest surface temperatures $\left(5000^{\circ} \mathrm{F}\right.$ on the nose portion of the vehicle) occurred at a moderate operating condition $\left(M_{\infty}=15, \sim 100,000\right.$ feet) along the proposed NASP ascent trajectory. The aerodynamic heating of the vehicle decreased the natural frequencies of the system by up to $30 \%$. Furthermore, it was observed that the cold vehicle experienced body freedom flutter, while the hot vehicle was primarily susceptible to elastic mode flutter. These results are illustrated in Fig. 34. In Ref. [92], the analysis of Ref. [53] was continued by including the effect of thermal stresses in the aerothermoelastic calculations. In this case, aerodynamic heating reduced the first six natural frequencies by $13-20 \%$, and lowered the flutter boundary by up to $25 \%$.

One of the main issues in performing an aerothermoelastic analysis is the need to combine several different computational tools, such as a CFD solver, a structural solver, and a heat transfer solver. Significant transfer of data between these components is required for advanced computational aerothermoelastic (CATE) analysis. A few researchers have focused on developing codes to efficiently combine these components. Reference [102] coupled the flow, thermal, and structural analyses into one integrated code, using the finite element method. The aerodynamic pressure and heating were determined by solving the Navier-Stokes equations. Results were generated for aerodynamically heated stainless steel panels, and indicated that at hypersonic Mach numbers $(M=6.6)$, panel deformations, due to aerodynamic heating and pressures, introduce shocks, expansions, and recirculation regions in the flow. Consequently, the heating rate distributions were altered significantly. In a related study, ${ }^{108}$ an implicit/explicit upwind cell-centered finite element algorithm, coupled with an adaptive unstructured finite element remeshing technique, was examined in order to study the fluid-thermal-structural interaction of aerodynamically heated leading edges. The analysis was validated with experimental results of a cylinder in hypersonic flow $(M=8.0)$, and applied to a 0.25 -inch diameter leading edge. Results indicated that deformations, due to non-uniform aerodynamic heating distributions significantly altered the flow field, and thus the aerothermal loads.

Reference [109] proposed solving the fluid-structural-thermal problem with a loosely coupled algorithm, which combined existing CFD, Computational Structural Dynamics (CSD) and Computational ThermoDynamics (CTD) codes. The authors chose the CFD code FEFLO98, the CSD codes COSMIC-NASTRAN 


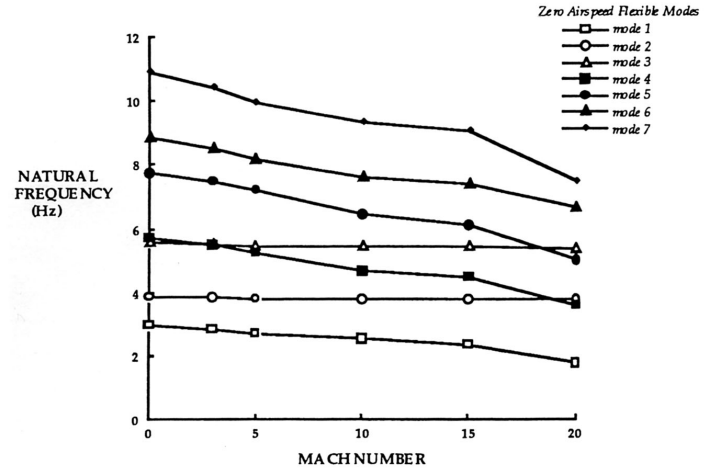

(a) Natural Frequencies

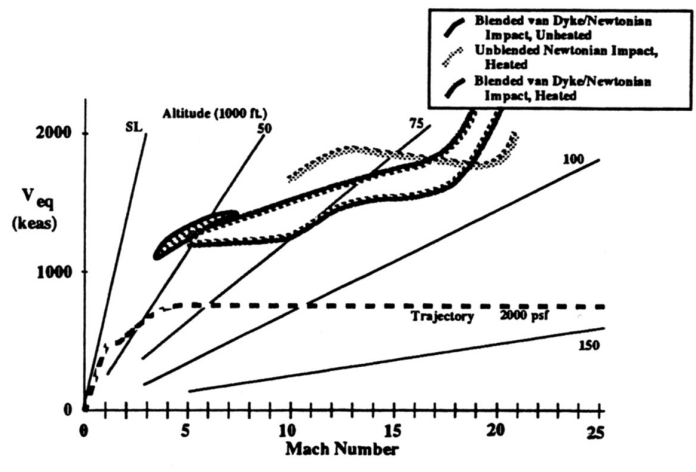

(b) Flutter Boundary

Figure 34. Effects of aerodynamic heating on the frequencies and flutter boundary of a NASP like hypersonic vehicle.[53]

for linear analysis and DYNA3D for nonlinear analysis, and the CTD code COSMIC-NASTRAN. A loose coupling of these codes was achieved by selecting a master surface for a specific variable, and interpolating/projecting that variable to the other codes at each time step. The algorithm provided a cost-effective means of using existing CFD, CSD, and CTD codes, with minimal alterations. The method was applied to several examples, including the aerodynamically heated panel configurations studied in Ref. [102].

Reference [65] used an integrated fluid-structure-thermal solver to perform analysis on the aerodynamic heating of an F-16 airfoil and an aerothermoelastic stability analysis of a flat panel. The aerothermoelastic formulation ${ }^{65}$ was an extension of a previous aeroelastic formulation developed in Ref. [60]. In the aerothermoelastic analysis, only one-way thermal coupling was considered. In this case, stress and deformations due to temperature changes are included, however feedback is neglected between the stress/deformations and the aerodynamic heating computations. The fluid, structure and mesh dynamics were solved separately in a serial manner, and the solutions from each computational domain were then transferred via the interface boundary, in order to account for interaction effects. It was observed that aerodynamically heating a panel reduces the flutter boundary and increases the amplitudes of oscillation.

Recently, the authors of this study were involved in developing a CATE methodology that Incorporated the heat transfer between the fluid and the structure using CFD-based aerodynamic heating computations. ${ }^{33,49}$ The CATE analysis procedure was then used to investigate the aerothermoelastic behavior of a low-aspect ratio wing in hypersonic flow. The CATE method of solution ${ }^{33,49}$ is shown in Fig. 35. Note that it is a modification to the original CAE solution procedure ${ }^{33,44-50}$ shown in Fig. 20. Thus, for the CATE analysis, the aerodynamic heating conditions were extracted from the CFD solver and passed to the finite element analysis module. Within the FEA module, shown in Fig. 35b) a heat transfer analysis was performed, and subsequently used to compute the heated free vibration modes and frequencies.

It was noted in Ref. [33] that the heat transfer on hypersonic vehicles is transient. Therefore, in regards to hypersonic aerothermoelasticity, an important consideration is the operating conditions through time. For instance, the transient heating at a constant altitude with constant Mach number is quite different than the transient heating with continuously changing operating conditions. Therefore, the transient heating of a hypersonic vehicle is inherently linked to its trajectory. In order to account for this, the aerothermoelastic stability of the wing was computed along a representative hypersonic trajectory, based on that proposed for the FALCON hypersonic cruise vehicle. ${ }^{33}$

As discussed in the introduction, the FALCON (Force Application and Launch from CONUS) program is intended to give the United States a global prompt strike capability by using hypersonic sub-orbital launch platforms. ${ }^{5}$ The long term goal of FALCON is an autonomous hypersonic cruise vehicle (HCV) that will take off like a conventional aircraft and transport 12,000 lbs. of cargo up to $17,000 \mathrm{~km}$ in less than 2 hours. The 
trajectory of the HCV consists of three phases. In the initial phase, the vehicle takes off from a conventional runway and is lifted from sea level to $40 \mathrm{~km}$, where the Mach number is a maximum at $M_{\infty}=12$. In the second phase the engines are turned off, however, the HCV continues to climb ballistically to an apogee of $60 \mathrm{~km}$. At this point the vehicle begins an equilibrium glide to an altitude of $35 \mathrm{~km}$. Here, the third phase begins as the engines re-ignite and push the vehicle back to an altitude of $40 \mathrm{~km}\left(M_{\infty}=12\right)$ to start the second phase again. This "skipping" type trajectory is proposed for the HCV in order to prevent heat build up by operating in a near-space environment. The representative trajectory, ${ }^{33}$ based on this proposed FALCON trajectory, is illustrated in Fig. 36.

Using the representative hypersonic trajectory, the variation in free vibration characteristics of the wing was determined. Two different cases were studied, in order to assess the sensitivity of the free vibration characteristics to relatively small variations in the trajectory. First, the free vibration characteristics were computed for the wing at varying angle-of-attack $\left(\alpha_{s}=0^{\circ}, 2^{\circ}\right.$, and $\left.4^{\circ}\right)$. The results from this analysis are presented in Fig. 37a). It is interesting that the change in frequencies, with aerodynamic heating, was not sensitive to variations in flow angle-of-attack. For the second case, the free vibration characteristics of the wing were calculated along the trajectory with the Mach number increased by $25 \%\left(\alpha_{s}=2^{o}\right)$. From Fig. 37b), it is evident that increasing the Mach number by $25 \%$ produced qualitatively similar results, however, the time to thermal buckling was reduced by approximately 8 minutes. It is also clear that, for this trajectory, increasing the Mach number by $25 \%$ had a more dramatic effect on the modal frequencies of the wing, than increasing the angle-of-attack from $0^{\circ}$ to $4^{\circ}$.

Figure 37c) illustrates the change in frequencies along the trajectory as a function of a reference temperature, $T_{R e f}$. Here, $T_{R e f}$ is the temperature at the leading edge of the $75 \%$ span section. ${ }^{33,49}$ It is apparent that when the frequencies are plotted as a function of reference temperature, there are only minimal differences between each case. This implies that while the heating rate changes by varying the the Mach number, the temperature distribution throughout the wing remains relatively constant. ${ }^{33}$ In other words, the time to buckling may vary with Mach number perturbations, however, the changes in the frequency, as a function of a reference temperature, are relatively unchanged.

The maximum pre-buckling changes in modal frequencies ${ }^{33}$ along the hypersonic trajectory are given in Table 5. Aerodynamic heating along the trajectory resulted in $20 \%-30 \%$ reductions in the second, third and fifth modal frequencies of the wing. In particular, the maximum reduction in stiffness occured for the first torsional mode (second modal frequency), which is a critical mode for flutter.

Table 5. Maximum percent change in the pre-buckled modal frequencies of the heated low-aspect ratio wing operating on a representative trajectory. A negative sign indicates a decrease in frequency. [33]

\begin{tabular}{cccccc}
\hline \hline Case & Mode 1 & Mode 2 & Mode 3 & Mode 4 & Mode 5 \\
\hline$\alpha_{s}=0^{\circ}$ & $-2.4 \%$ & $-31.0 \%$ & $-16.9 \%$ & $5.8 \%$ & $-24.4 \%$ \\
$\alpha_{s}=2^{\circ}$ & $-2.8 \%$ & $-30.8 \%$ & $-17.3 \%$ & $5.3 \%$ & $-24.7 \%$ \\
$\alpha_{s}=4^{o}$ & $-1.2 \%$ & $-30.6 \%$ & $-16.6 \%$ & $6.2 \%$ & $-24.7 \%$ \\
Increased Mach No. & $-2.1 \%$ & $-31.2 \%$ & $-17.6 \%$ & $5.6 \%$ & $-25.3 \%$ \\
\hline \hline
\end{tabular}

Using the time dependent free vibration characteristics, the aerothermoelastic behavior of the wing was investigated. ${ }^{33}$ In order to perform this analysis, however, several issues had to be addressed first. For instance, note that when heating is neglected the structural properties are independent of operating conditions. Therefore, flutter is calculated by holding altitude constant, and increasing Mach number until critical damping of the system occurs. In an aerothermoelastic solution, however, aerodynamic heating couples the structural properties to the operating conditions. Therefore, the structure must be updated according to the operating conditions at the current point in the trajectory. Similar to the unheated case, flutter can be calculated by holding altitude constant and increasing the Mach number. However, this is a virtual flutter Mach number, since the structural properties are not updated at each increase in Mach number to critical system damping. Therefore, this only yields a quantitative measure of the proximity to flutter, and not the actual flutter Mach number. For this reason, it is important to check the sensitivity of the virtual flutter Mach number to perturbations in the trajectory, such as modest increases to the trajectory operating Mach number. ${ }^{33}$

A second issue is the operation of the wing at high altitudes, for the representative trajectory shown in 


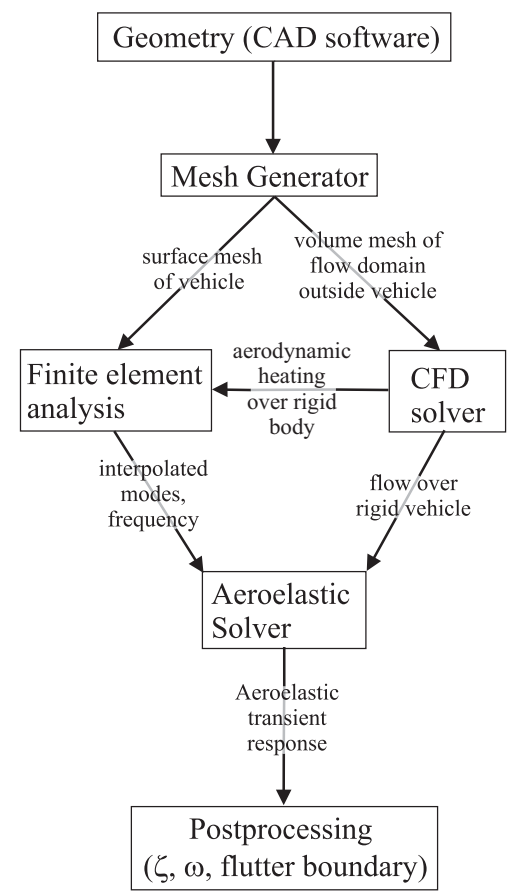

(a) Aerothermoelastic Solution

Procedure.

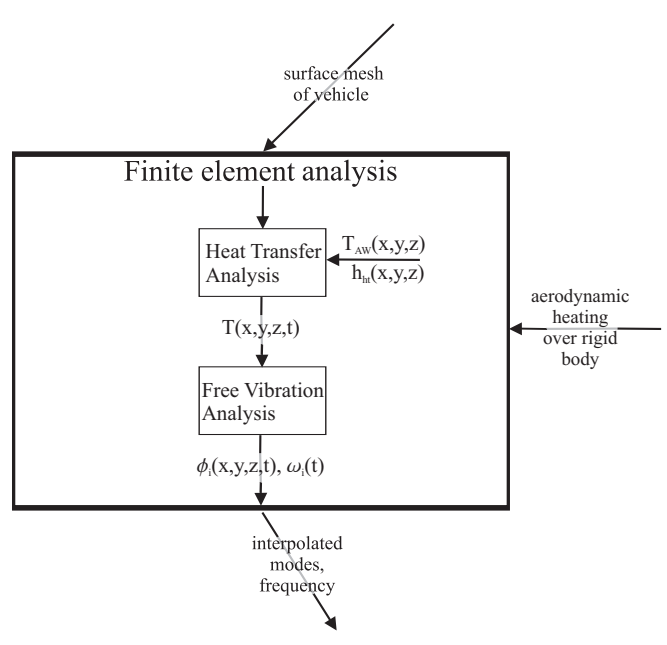

(b) Finite Element Analysis.

Figure 35. A flow diagram for the computational aerothermoelastic solution procedure. The aerodynamic heating information is passed from the CFD solver to the finite element analysis module; in order to compute the heat transfer between the fluid and structure, and heated free vibration characteristics.[33, 49]

Fig. 36. As illustrated in Fig. 27, the wing fluttered at very high Mach numbers, at altitudes relatively low compared to most portions of the representative hypersonic trajectory. Thus, since the wing was assumed to operate at such high altitudes, the virtual flutter Mach numbers were unrealistic. Therefore, at each operating condition in the trajectory, the Mach number and altitude were held constant, and only the dynamic pressure was increased until flutter occurred. Using this method, the proximity to flutter was 

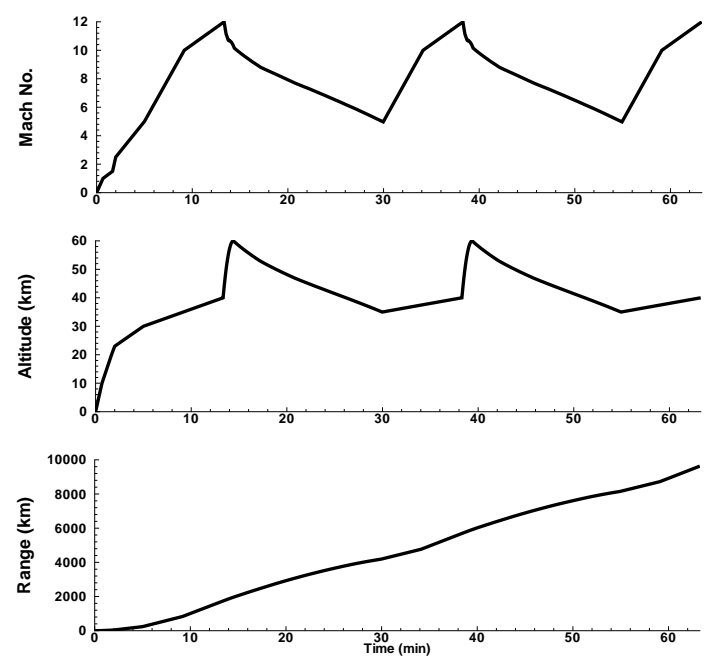

Figure 36. Representative trajectory, based on the FALCON program, of a hypersonic vehicle.[33]

determined by calculating the ratio of the virtual flutter dynamic pressure, $q_{v f}$, to the free-stream dynamic pressure, $q_{\infty}$. A value of unity for this ratio at a given point on the trajectory implies the wing flutters at that operating condition.

The aerothermoelastic behavior of the wing along the trajectory is illustrated in Fig. 38. Due to the agreement between the aeroelastic behavior predicted using third order piston theory and CFD-based aerodynamics at high altitudes and Mach numbers, as illustrated in Fig. 27, the majority of aerothermoelastic computations for the modified wing were carried out using third order piston theory unsteady aerodynamics. However, for verification purposes, the aerothermoelastic behavior was computed using Euler unsteady aerodynamics at a few points on the trajectory. Several important conclusions were made from these results. First, it is clear by comparing the heated and unheated dynamic pressure ratios of the $0^{\circ}$ angle-of-attack cases, that prior to thermal buckling, aerodynamic heating reduces the flutter margin by up to $40 \%$. However, as expected, for most portions of the trajectory, the virtual flutter dynamic pressure were significantly higher than the free-stream dynamic pressure, for all cases considered. In particular, for the entire unheated case, and first 30 minutes of the heated cases, the virtual flutter dynamic pressure were two to three orders of magnitude higher than the free-stream dynamic pressure. Furthermore, the minimum dynamic pressure ratios were approximately $\mathcal{O}(10)$. These minimum ratios occurred after the first skip phase, and during the re-acceleration phase where the altitudes are relatively moderate and the Mach numbers are maximum. It is also evident from these results that angle-of-attack did not significantly alter the flutter margin of the wing. Furthermore, increasing the Mach number by $25 \%$ resulted in approximately a $30 \%$ reduction in the dynamic pressure ratio in the pre-buckled portion of the trajectory. Finally, note that, as in the unheated case, there were only minimal differences between the Euler and third order piston theory results.

It is apparent from the large dynamic pressure ratios that, for this type of trajectory, thermal buckling is more critical to the wing than flutter. ${ }^{33}$ This is likely due to the low density air present at the near-space environment of the representative trajectory. These results emphasize the need for advanced materials and/or an active cooling system, since the wing fails well before the $17,000 \mathrm{~km}$ desired range of the HCV. Specifically, for this trajectory and configuration, the wing buckled after approximately 7,000 km (45 minutes). ${ }^{33}$

\section{Future Directions}

From this review of the current status of hypersonic aeroelasticity and aerothermoelasticity, a number of future directions can be identified. First, it is clear that sophisticated computational methodologies are becoming more prevalent in the field of aeroelasticity. Aeroelasticians now have the ability to implement unsteady aerodynamics based on solutions to the unsteady Euler and Navier-Stokes equations. However, there are still many steps between fundamental studies using these tools, and full-scale aeroelastic analysis 


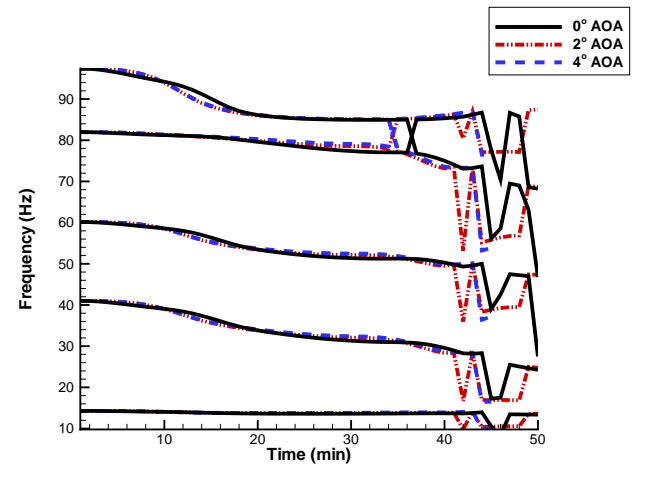

(a) Variation of frequency vs. time for different Angles of Attack

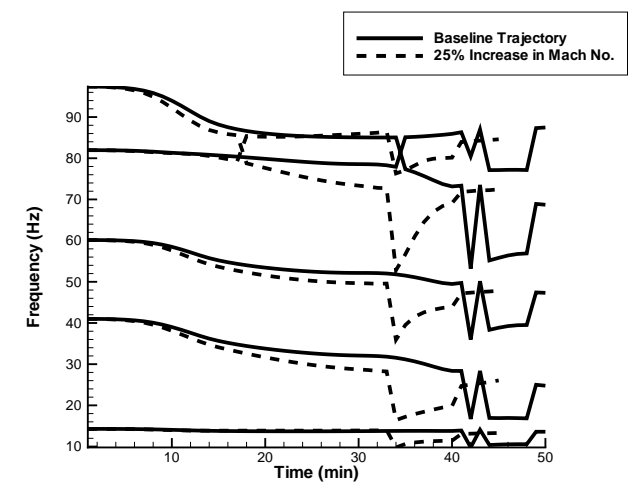

(b) Effect of increasing the trajectory Mach number by $25 \% . \alpha_{s}=2^{\circ}$

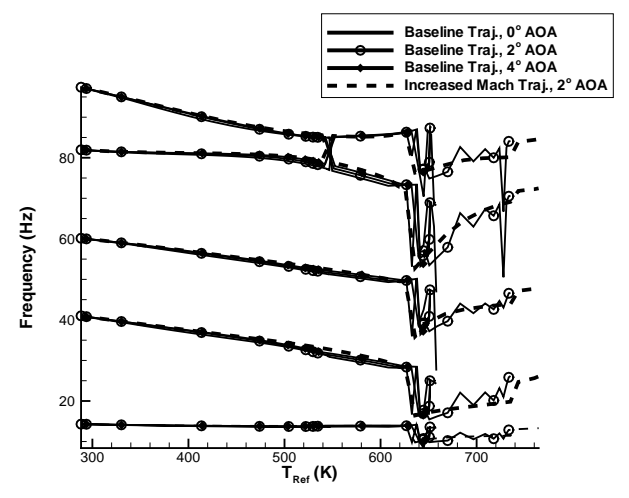

(c) Variation of frequency vs. $T_{R e f}$

Figure 37. Natural frequencies of the modified low-aspect ratio wing operating along a representative hypersonic trajectory. [33]

and design of hypersonic vehicles. The biggest obstacle is the difficulty, and extreme computational cost, of incorporating aerodynamic heating. As shown, there has been limited work in this area, and results produced have been at the fundamental level. Therefore, much work is needed in this area, to: 1) gain a complete understanding of the important aspects, and 2) develop computationally feasible methodologies that address these aspects. Incorporating real gas effects and chemistry in an accurate manner in the aerothermoelastic analysis is another important consideration that is required in order to design hypersonic vehicles in a reliable manner.

Other future directions in hypersonic aeroelasticity and aerothermoelasticity deal with the multi-disciplinary nature of hypersonic vehicles. As discussed earlier, the way hypersonic vehicles are configured results in significant interactions between the structural, control, propulsion, and lifting components. ${ }^{11-13}$ Therefore, aerothermoelasticity is not only an important consideration for the structural system, but also for the propulsion and control systems. During the last 15 years, a number of studies have focused on the multidisciplinary analysis of hypersonic vehicles. However, as will be seen from the review of these studies below, the analysis has invariably implemented extensive simplification of the actual problem. 

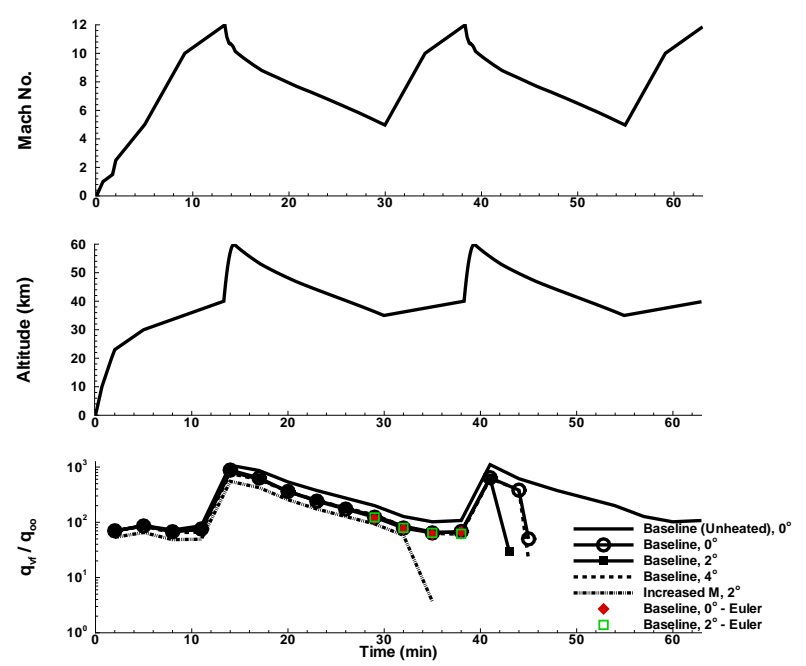

Figure 38. Aerothermoelastic flutter margin of the modified low-aspect ratio wing along a representative hypersonic trajectory.[33]

\section{A. Dynamics and Control of Hypersonic Vehicles}

Recent multi-disciplinary research into the dynamics and control of air-breathing hypersonics has mainly focused on configurations resembling the conceptual National Aerospace Plane (NASP) and NASA experimental X-43 aircraft. Each of these studies has implemented system models of varying complexity, in order to study the effects of the integrated propulsion-airframe design on vehicle dynamics and control.

Reference [110] investigated a simplified, elastic hypersonic vehicle resembling the NASP X-30 configuration, assumed to operate at Mach 8 and 85,000 ft altitude. The airframe aerodynamics were predicted using 2-D Newtonian flow assumptions. Thus, viscous and aeroheating effects were ignored. It was concluded that a holistic approach is required in order to synthesize the airframe/engine control system. A second study on the X-30 NASP demonstrator configuration investigated active control of the vehicle when aerothermoelastic effects are included. ${ }^{92}$ The structure was modeled using the finite element method, while the generalized forces were computed using Van Dykes second order unsteady aerodynamics. The aerodynamic heating was computed using the Aerodynamic Preliminary Analysis System code (APAS) ${ }^{107}$ and changes in the structural frequencies and mode shapes were determined using a finite element heat transfer and free vibration analysis. It was concluded that aerodynamic heating significantly lowered the vehicle flutter boundary; however the boundary was subsequently improved via active control.

Reference [111] investigated the development of uncertainty models for multi-variable control robustness analysis of an air-breathing hypersonic vehicle resembling the X-30 NASP demonstrator. It was noted that important sources of uncertainty are the vehicles structural dynamic characteristics and aerodynamic loads. Furthermore, the flexible degrees of freedom were found to be quite important for flight-control synthesis of air-breathing hypersonic vehicles.

References $[112,113]$ implemented a simple aerothermoelastic model in order to study control issues of air-breathing hypersonic vehicles. In Ref. [112], a linear parameter-varying framework was used to account for aerothermoelastic effects on a hypersonic vehicle. In a follow up study, ${ }^{113}$ a multi-loop aeroservoelastic controller was developed to both augment damping in the structural modes and control rigid body dynamics. The aerothermoelastic model was created by assuming reductions in the free vibration frequencies of the X-30 structural model. The assumed reductions were estimated using Ref. [53].

Reference [114] investigated the dynamics of hypersonic wave riders at Mach 10, and an altitude of 30 $\mathrm{km}$. A finite difference method was used to model the aerodynamic pressures, while the Eckert reference temperature method ${ }^{10}$ was used to compute the drag. It was concluded that the propulsion effects must be accurately modeled in order to develop the system controller.

In a recent study ${ }^{115}$ it is noted that there is a lack of complete models that adequately include and quantify the unique characteristics of air-breathing hypersonic vehicles. A review of the integrated problem 
is provided, and the development of a high-fidelity CFD-based model of a full scale hypersonic vehicle is discussed. As a first step towards this goal, a simple two-dimensional geometry is studied at Mach 10, and an altitude of $30 \mathrm{~km}$. The SCRamjet combustion process was modeled using one-dimensional flow with heat addition. The CFD data was generated using Fluent, assuming an ideal gas. In another recent study, ${ }^{116}$ a comprehensive, non-linear model of the integrated system was developed that accounted for vehicle aerodynamics, propulsion, and structural dynamics. The aerodynamic pressures were computed using Shock-Expansion theory, while the structural dynamics were modeled using a simple joined-beam configuration. The propulsion system was modeled assuming a one-dimensional flow with heat addition.

\section{B. Aerothermoelastic Effects on the Performance of an Integrated Airframe-Propulsion Ve- hicle}

The impact of aeroelasticity on the performance of an air-breathing propulsion system, for a configuration resembling the NASP, was investigated in Ref. [90]. The structural dynamics and response were computed using the free vibration modes of the structure, the propulsion model was developed using the SRGULL hypersonic propulsion code, ${ }^{117}$ and the unsteady aerodynamics were computed using second order piston theory. The propulsion model exhibited a pronounced sensitivity to angle-of-attack and elastic fuselage deflections. Furthermore, this had a significant impact on the vehicle rigid body flight dynamics.

It is noted in Ref. [118] that maximum system efficiency is critical to successful mission operations of airbreathing hypersonic vehicles. Therefore, maximizing efficiency of the highly coupled subsystems is essential. Using a relatively simple system model, the generated results indicated that the maximum achievable orbital mass fraction is a strong function of propulsion system-efficiencies. This, in conjunction with Ref. [90], emphasizes the importance of accurately modeling the vehicle structure. Also, heating constraints were found to have a significant effect on operation efficiency and mission performance.

A high-fidelity CFD analysis of the aero-propulsive performance, i.e. interaction between the aerodynamics and propulsion systems, of the X-43 was described in Ref. [119]. Furthermore, an overview on the methods used in the analysis and preflight database development for the X-43 experimental flights was provided. The GASP code ${ }^{120}$ was the primary CFD code used in the X-43 preflight performance analysis. This code is a Navier-Stokes solver capable of modeling frozen, equilibrium or finite rate chemistry with models for hydrogen-air combustion. The internal analysis of the propulsion flow path was carried out using the SHIP $^{121,122}$ and SGRULL ${ }^{117}$ codes. Note that the effect of the structure was not included in the analysis.

Reference [123] investigated the development and implementation of a multi-disciplinary design optimization (MDO) procedure that coupled the propulsion, aerodynamics, mass properties, and vehicle volume. The MDO process was implemented so as to geometrically optimize a hypersonic cruise missile for maximum overall mission range. In the study, the aerodynamic pressures were computed using Shock-Expansion theory, while the viscous drag was estimated from a table look-up based on wetted area, Mach number, and operating altitude. During the design process, the author noted that the optimization results were found to be counter to that obtained using uncoupled, single-variable-at-a-time trade studies.

In order to study the fluid-thermal-structural interactions in an air-breathing hypersonic engine, Ref. [124] used off-the-shelf solvers to loosely couple the fluid, chemistry, thermal, and structural environments. The hypersonic flow over the vehicle fore body and into the engine inlet was computed using OVERFLOW, ${ }^{125}$ the combustor fluid-chemistry analysis was simulated using VULCAN, ${ }^{126}$ and the engine strut thermal and structural analyses were computed using ANSYS. ${ }^{127}$ Using this methodology, quantitative results were generated for inlet performance, engine combustion, heat transfer and thermal management, and structural deflections/stresses. It was noted, however, that the effort to setup the single discipline simulations, for comprehensive analysis of the engine only, was on the order of months. Furthermore, the process is complicated by the effort required to couple the single discipline computations, both due to manual labor of transferring the relevant input/output between the codes and the disparate execution times for the different component solvers.

In Ref. [128], a comprehensive, computational aero-propulsive analysis of a full scale hypersonic vehicle was performed as a follow up to Ref. [115]. In particular, simulations were carried out using the Fluent CFD code in order to construct a complete set of aerodynamic and coupled aero-propulsion data for the vehicle. At conclusion of the study, it was noted that the hypersonic control law design is associated with a large of amount of system uncertainty due to ignored coupling effects. It is concluded that accurate simulation models are needed for the inclusion of system coupling into the comprehensive analysis.

It is clear from the studies presented above on the multi-disciplinary analysis of hypersonic vehicles 
that, for comprehensive analysis and design of these complex vehicles, an accurate structural model is required. Indeed, many of the researchers in these studies have shown this to be true through comprehensive analysis using simplified models. Thus, since the structure is strongly influenced by aerothermoelastic effects, much work is left to be done to refine the current state-of-the-art in hypersonic aerothermoelasticity, and incorporate it into holistic analysis procedures.

\section{Conclusions}

The history of hypersonic flight research spans over six decades; however, we are still far from the regular and sustained flight of aircraft in this flow regime. In particular, the airframe is a system that continues to pose a significant obstacle to the development of hypersonic vehicles. Aerodynamic heat and pressure loads are extreme within the hypersonic flight regime. This, in conjunction with minimum weight restrictions, implies that careful design of the airframe is necessary in order to survive this harsh environment. However, the study of hypersonic aerothermoelasticity is not only necessary for vehicle survivability, but also for the development of the propulsion and control systems; since airbreathing hypersonic configurations exhibit tight coupling between the airframe and these vehicle components. Therefore, in order to fully understand these interactions, imaginative research is needed to incorporate the effect of hypersonic aerothermoelasticity into the multi-disciplinary analysis of hypersonic vehicles. Complicating the issue is the inability to test aeroelastically and aerothermoelastically scaled models. Thus, computational aeroelasticity and aerothermoelasticity are essential to this flight regime.

There have been several important recent advances in CAE and CATE, namely: 1) the development of CFD-based computational aeroelastic codes; 2) the study of fundamental issues involving the use of CAE/CATE in the hypersonic flow regime; and 3) the use of CFD-based aerodynamic heating computations for the study of the aerothermoelastic problem. However, it is clear that issues such as: 1) the effect of viscosity; 2) the incorporation of real gas effects; and 3) the accurate and efficient incorporation of CFDbased aerodynamic heating, will continue to be critical research areas for the development of hypersonic vehicles.

The usefulness of hypersonic vehicles, for both military and civilian purposes, is unquestionable. However, it is clear that the state-of-the-art in the area of hypersonic aerothermoelasticity is far from sufficient for the design of hypersonic vehicles.

\section{Acknowledgments}

This research was supported by both the Space Vehicle Technology Institute under grant NCC3-989 jointly funded by NASA and DOD within the NASA Constellation University Institutes Project, with Claudia Meyer as the project manager; and Air Force Grant F49620-01-0158 with Dr. Clark Allred as program manager.

\section{References}

1) Anderson, J.D., Hypersonic and High Temperature Gas Dynamics, McGraw-Hill, New York, 1989.

2) Nydick, I., Studies in Hypersonic Aeroelasticity, Ph.D. thesis, University of California, Los Angeles, 2000.

3) McClinton, C., "X-43 Scramjet Power Breaks the Hypersonic Barrier: Dryden Lectureship in Research for 2006," 44th AIAA Aerospace Sciences Meeting and Exhibit, Reno NV, 2006, AIAA 2006-1.

4) Neuenhahn, T., Olivier, H., and Paull, A., "Development of the Hyshot Stability Demonstrator," 25th AIAA Aerodynamic Measurement Technology and Ground Testing Conference, San Francisco CA, 2006, AIAA 2006-2960.

5) Walker, W. and Rodgers, F., "Falcon Hypersonic Technology Overview," 13th International Space Planes and Hypersonic Systems and Technologies Conference, Capua Italy, 2005, AIAA 2005-3253.

6) Ricketts, R., Noll, T., Whitlow, W., and Huttsell,L., "An Overview of Aeroelasticity Studies for the National Aerospace Plane," Proc. 34th AIAA/ASME/ASCE/AHS/ASC Structures, Structural Dynamics and Materials Conference, La Jolla, CA, April 19-22 1993, pp. 152 - 162, AIAA Paper 93-1313.

7) Gupta, K.K., Voelker, L.S., Bach, C., Doyle, T., and Hahn, E., "CFD-Based Aeroelastic Analysis of the X-43 Hypersonic Flight Vehicle," 39th Aerospace Sciences Meeting \& Exhibit, 2001, AIAA Paper No. 2001-0712.

8) Berry, S.A., Horvath, T.J., Hollis, B.R., Thompson, R.A., and Hamilton, H.H., "X-33 Hypersonic Boundary Layer Transition," 33rd AIAA Thermophysics Conference, Norfolk, VA, June 28 - July 1 1999, AIAA Paper 99-3560.

9) Riley, C.J., Kleb, W.L., and Alter, S.J., "Aeroheating Predictions for X-34 Using An Inviscid-Boundary Layer Method," 36th Aerospace Sciences Meeting \& Exhibit, Reno, NV, January 1998, AIAA Paper 98-0880.

10) Bertin, J.J., Hypersonic Aerothermodynamics, AIAA, 1994. 
11) Blankson, IM., "Air-Breathing Hypersonic Waveriders: A Survey of Research Needs," Proceedings of the First International Waverider Symposium, University of Maryland, College Park, MD, October 1990.

12) Bertin, J.J. and Cummings, R.M., "Fifty Years of Hypersonics: Where We've Been and Where We're Going," Progress in Aerospace Sciences, Vol. 39, April 2003, pp. 511-536.

13) Fidan, B., Mirmirani, M., and Ioannou, P., "Flight Dynamics and Control of Air-Breathing Hypersonic Vehicles: Review and New Directions," 12th AIAA International Space Planes and Hypersonic Systems and Technologies, Norfolk, VA, 2003, AIAA Paper No. 2003-7081.

14) Bisplinghoff, R. and Ashley, H., Principles of Aeroelasticity, John Wiley \& Sons, New York, 1962.

15) Bisplinghoff, R.L., Ashley, H., and Halfman, R.L., Aeroelasticity, Addison-Wesley, 1955.

16) Friedmann, P.P., "Renaissance of Aeroelasticity and Its Future," Journal of Aircraft, Vol. 36, No. 1, Jan/Feb 1999, pp. 105-121.

17) Bisplinghoff, R.L. and Dugundji, J., Influence of Aerodynamic Heating on Aeroelastic Phenomena, Pergamon Press, 1958, pp. 288-312.

18) Lauten, W.T., Levey, G.M., and Armstrong, W.O., "Investigation of an All-Movable Control Surface at a Mach Number of 6.86 For Possible Flutter," NACA RM L58B27, 1958.

19) Dogget, R.V., "Experimental Flutter Investigation of Some Simple Models of a Boost-Glide-Vehicle Wing at Mach Numbers of 3.0 and 7.3," NASA TM X-37, 1959.

20) Miller, R.W. and Hannah, M.E., "Flutter Investigation of 60 deg to 80 deg Delta-Planform Surfaces at a Mach Number of 7.0," NASA TM X-325, 1960.

21) Hanson, P.W., "Aerodynamic Effects of Some Configuration Variables on the Aeroelastic Characteristics of Lifting Surfaces at Mach Numbers From 0.7 to 6.86," NASA TN D-984, 1961.

22) Sewall, J.L., Hess, R.W., and Watkins, C.E., "Analytical and Experimental Investigation of Flutter and Divergence of Spring-Mounted Cone Configurations at Supersonic Speeds," NASA TN D-1021, 1962.

23) Young, L.S., "Effects of Angle of Attack and Thickness Ratio on the Flutter of a Rigid Unswept Diamond-AirfoilSection Wing at a Mach Number of 10.0," NASA TN D-1380, 1962.

24) Goetz, R.C., "Effects of Leading-Edge Bluntness on Flutter Characteristics of Some Square-Planform Double-Wedge Airfoils at a Mach Number of 15.4," NASA TN D-1487, 1962.

25) Garrick, I.E., "A Survey of Aerothermoelastic," Aerospace Engineering, January 1963, pp. 140-147.

26) Hedgepeth, J. and Widmayer, E., "Dynamic and Aeroelastic Problems of Lifting Re-Entry Bodies," Aerospace Engineering, January, 1963, pp. 148-153.

27) Laidlaw, W.R. and Wyker, J.H., "Potential Aerothermoelastic Problems Associated with Advanced Vehicle Design," Aerospace Engineering, January, 1963, pp. 154-164.

28) Goetz, R.C., "Effects of Leading-Edge Sweep on Flutter Characteristics of Some Delta-Planform Surfaces at a Mach Number of 15.4," NASA TN D-2360, 1964.

29) Goetz, R.C. and Sewall, J.L., "Experimental Flutter Study of a Wing-Fuselage Configuration at a Mach Number of 15.4 and Comparison With Theory," NASA TN D-3046, 1965.

30) Goetz, R.C., "Hypersonic Flutter Analysis Using Measured Static Aerodynamic Derivatives, and Comparison with Experiment," NASA TN D-5233, 1969.

31) Rasmussen, M., Hypersonic Flow, John Wiley \& Sons, New York, 1994.

32) Mei, C., Abdel-Motagly, K., and Chen, R., "Review of Nonlinear Panel Flutter at Supersonic and Hypersonic Speeds," Applied Mechanics Reviews, 1998.

33) McNamara, J., Aeroelastic and Aerothermoelastic Behavior of Two and Three Dimensional Surfaces in Hypersonic Flow, Ph.D. thesis, University of Michigan, Ann Arbor, 2005.

34) Lighthill, M.J., "Oscillating Airfoils at High Mach Numbers," Journal of the Aeronautical Sciences, Vol. 20, No. 6, June 1953.

35) Ashley, H. and Zartarian, G., "Piston Theory - A New Aerodynamic Tool for the Aeroelastician," Journal of the Aeronautical Sciences, Vol. 23, No. 12, 1956, pp. 1109-1118.

36) Van Dyke, M. D.,, "A Study of Second-Order Supersonic Flow Theory," Tech. Rep. 1081, NACA, 1951.

37) Morgan, H. G., Huckel, V., and Runyan, H. L., "Procedure for Calculating Flutter at High Supersonic Speed Including Camber Deflections, and Comparison with Experimental Results," NACA TN 4335, 1958.

38) Zartarian, G., Hsu, P. T., and Ashley, H., "Dynamic Airloads and Aeroelastic Problems at Entry Mach Numbers," Journal of Aeronautical Sciences, Vol. 28, March 1961, pp. 209 - 222.

39) Morgan H.G., Runyan H.L., and Huckel V., "Theoretical Considerations of Flutter at High Mach Numbers," Journal of the Aeronautical Sciences, Vol. 25, No. 6, 1958, pp. 371-381.

40) Yates, E.C. and Bennett, R.M., "Analysis of Supersonic-Hypersonic Flutter of Lifting Surfaces at Angle of Attack," Proc. 12th AIAA/ASME Structures, Structural Dynamics and Materials Conference, Anaheim, CA, April 1971, AIAA Paper No. 71-327.

41) Nydick, I., Friedmann, P.P., and Zhong, X., "Hypersonic Panel Flutter Studies on Curved Panels," Proc. 36th AIAA/ASME/ASCE/AHS/ASC Structures, Structural Dynamics and Materials Conference, New Orleans, LA, April 1995, pp. 2995-3011, AIAA Paper 95-1485.

42) Spain, C., Zeiler, T.A., Bullock, E., and Hodge, J.S., "A Flutter Investigation of All-Moveable NASP-Like Wings at Hypersonic Speeds," Proc. 34th AIAA/ASME/ASCE/AHS/ ASC Structures, Structural Dynamics and Materials Conference, La Jolla, CA, April 19-22 1993, AIAA Paper No. 93-1315.

43) Liu, D. D., Yao, Z. X., Sarhaddi, D., and Chavez, F., "From Piston Theory to a Unified Hypersonic-Supersonic Lifting Surface Method," Journal of Aircraft, Vol. 34, No. 3, May-June 1997, pp. $304-312$. 
44) Thuruthimattam, B.J., Friedmann, P.P., McNamara, J.J., and Powell, K.G., "Aeroelasticity of a Generic Hypersonic Vehicle," Proc. 43rd AIAA/ASME/ASCE/AHS Structures, Structural Dynamics and Materials Conference, Denver, CO, April 2002, AIAA Paper No. 2002-1209.

45) Thuruthimattam, B.J., Friedmann, P.P., McNamara, J.J., and Powell, K.G., "Modeling Approaches to Hypersonic Aeroelasticity," Proc. IMECE'02, 2002 ASME International Mechanical Engineering Congress and Exposition, No. IMECE 2002-32943, New Orleans, LA, November 2002.

46) Thuruthimattam, B.J., Friedmann, P.P., McNamara, J.J., and Powell, K.G., "Modeling Approaches to Hypersonic Aerothermoelasticity with Application to Reusable Launch Vehicles," Proc. 44th AIAA/ASME/ASCE/AHS Structures, Structural Dynamics and Materials Conference, Norfolk, VA, April 2003, AIAA Paper No. 2003-1967.

47) McNamara, J.J., Thuruthimattam, B.J., Friedmann, P.P., Powell, K.G., and Bartels, R.E., "Hypersonic Aerothermoelastic Studies for Reusable Launch Vehicles," Proc. 45th AIAA/ASME/ASCE/AHS Structures, Structural Dynamics and Materials Conference, Palm Springs, CA, April 2004, AIAA Paper No. 2004-1590.

48) Friedmann, P.P., McNamara, J.J., Thuruthimattam, B.J., and Nydick, I., "Aeroelastic Analysis of Hypersonic Vehicles," J. of Fluids and Structures, Vol. 19, 2004, pp. 681-712.

49) McNamara, J.J., Friedmann, P.P., Powell, K.G., Thuruthimattam, B.J., and Bartels, R.E., "Three-dimensional Aeroelastic and Aerothermoelastic Behavior in Hypersonic Flow," Proc. 46th AIAA/ASME/ASCE/AHS Structures, Structural Dynamics and Materials Conference, Austin, TX, April 2005, AIAA Paper No. 2005-2175.

50) Thuruthimattam, B.J., Fundamental Studies in Hypersonic Aeroelasticity Using Computational Methods, Ph.D. thesis, University of Michigan, 2005.

51) Hayes, W. D. and Probstein, R. F., Hypersonic Flow Theory, Academic Press, Inc., 1st ed., 1959.

52) Anderson, J.D., Modern Compressible Flow, with Historical Perspective, McGraw-Hill, New York, 1990.

53) Heeg, J., Zeiler, T., Pototzky, A., Spain, C., and Engelund, W., "Aerothermoelastic Analysis of a NASP Demonstrator Model," Proc. 34th AIAA/ASME/ASCE/AHS/ ASC Structures, Structural Dynamics and Materials Conference, La Jolla, CA, April 19-22 1993, pp. 617-627, AIAA Paper No. 93-1366.

54) Cox, R. N. and Crabtree, L. F., Elements of Hypersonic Aerodynamics, Academic Press, 1965.

55) Tezduyar, T.E., Behr, M., and Liou, J., "A New Strategy for Finite Element Computations involving Moving Boundaries and Interfaces - The Deforming-spatial-domain/Space-time Procedure: I. The concept and the Preliminary Numerical Tests," Computer Methods in Applied Mechanics and Engineering, Vol. 94, 1992, pp. 339-351.

56) Tezduyar, T.E., Behr, M., and Liou, J., "A New Strategy for Finite Element Computations involving Moving Boundaries and Interfaces - The Deforming-spatial-domain/Space-time Procedure: II. Computation of Free-surface Flows, Two-liquid Flows, and Flows with Drifting Cylinders," Computer Methods in Applied Mechanics and Engineering, Vol. 94, 1992, pp. 353371.

57) Hughes, T.J.R. and Hulbert, G.M., "Space-Time Finite Element Methods For Elastodynamics: Formulations and Error Estimates," Computer Methods in Applied Mechanics and Engineering, Vol. 66, 1988, pp. 339-363.

58) Donea, J., Guiliani, S., and Halleux, J.P., "An Arbitrary Lagrangian-Eulerian Finite Element Method for Transient Dynamic Fluid-Structure Interactions," Computer Methods in Applied Mechanics and Engineering, Vol. 33, 1982 , pp. 689-723.

59) Bendiksen, O.O., "A New Approach to Computational Aeroelasticity," Proc. AIAA/ASME/ASCE/AHS/ASC 32nd Structure, Structural Dynamics and Materials Conf., Baltimore, MD, April 8-9 1991, pp. 1712-1727, AIAA Paper 91-0939.

60) Farhat, C., Lesoinne, M., and Maman, N., "Mixed Explicit/Implicit Time Integration of Coupled Aeroelastic Problems: Three-field Formulation, Geometric Conservation and Distributed Solution," International Journal for Numerical Methods in Fluids, Vol. 21, 1995, pp. 807-835.

61) Batina, J.T., "Unsteady Euler Airfoil Solutions Using Unstructured Dynamic Meshes," AIAA Journal, Vol. 28, 1990, pp. 1381-1388.

62) Rausch, R.D., Yang, H.T.Y., and Batina, J.T., "Euler Flutter Analysis of Airfoils Using Unstructured Dynamic Meshes," Journal of Aircraft, Vol. 27, No. 5, 1990, pp. 463-443.

63) Robinson, B.A., Batina, J.T., and Yang, H.T., "Aeroelastic Analysis of Wings Using the Euler Equations with a Deforming Mesh," Journal of Aircraft, Vol. 28, November 1991, pp. 778-788.

64) Bartels, R.E., "Mesh Strategies for Accurate Computation of Unsteady Spoiler and Aeroelastic Problems," Journal of Aircraft, Vol. 37, No. 3, 2000, pp. 521-525.

65) Tran H. and Farhat, C., "An Integrated Platform for the Simulation of Fluid-Structure-Thermal Interaction Problems," Proc. 43rd AIAA/ASME/ASCE/AHS Structures, Structural Dynamics and Materials Conference, Denver, CO, April 2002, AIAA Paper No. 2002-1307.

66) Bartels, R.E., Rumsey, C.L., and Biedron, R.T., "CFL3D Version 6.4 - General Usage and Aeroelastic Analysis," NASA TM -2006-214301, 2006.

67) Bartels, R.E., "Finite Macro-Element Mesh Deformation in a Structured Multi-Block Navier-Stokes Code," NASA TM -2005-213789, 2005.

68) Stephens, C.H., Arena Jr., A.S., and Gupta, K.K., "Application of the transpiration method for aeroservoelastic prediction using CFD," Proceedings of the 39th AIAA/ASME/ASCE/AHS/ASC Structures, Structural Dynamics, and Materials Conference and Exhibit, Long Beach, CA, April 20-23 1998, AIAA Paper 98-2071.

69) Hartwich, P. and Agrawal, S., "Method For Perturbing Multiblock Patched Grids in Aeroelastic Design Optimization Applications," June 1997, AIAA Paper 97-2038.

70) Thompson, J., Bharat, S., and Weatherrill, N., Handbook of Grid Generation, CRC Press, Boca Raton, FL, 1998.

71) Krist, S.L., Biedron, R.T., and Rumsey, C.L., "CFL3D User's Manual (Version 5.0)," NASA TM 1998-208444, 1997.

72) Dowell, E.H., Aeroelasticity of Plates and Shells, Noordhoff International Publishing, Lyden, The Netherlands, 1975.

73) Bein, T., Friedmann, P., Zhong, X., and Nydick, I., "Hypersonic Flutter of a Curved Shallow Panel with Aerodynamic Heating," Proc. 34th AIAA/ASME/ASCE/AHS/ASC Structures, Structural Dynamics and Materials Conference, La Jolla, CA, April 19-22 1993, AIAA Paper 93-1318. 
74) Mei, C. and Grey, C., "A Finite Element Method for Large-Amplitude, Two-Dimensional Panel Flutter at Hypersonic Speeds," Proc. 30th AIAA/ASME/ASCE/AHS/ASC Structures, Structural Dynamics and Materials Conference, Mobile, AL, April 1989, pp. 37-71, AIAA Paper No. 89-1165.

75) Hedgepeth, J.M., "Flutter of Rectangular Simply Supported Panels at High Supersonic Speeds," Journal of the Aeronautical Sciences, Vol. 24, No. 8, August 1957, pp. 563-573.

76) Dowell, E.H., "Nonlinear Oscillations of a Fluttering Plate," AIAA J, Vol. 4, No. 7, 1966, pp. 1267-1275.

77) Friedmann, P. and Hanin, M., "Supersonic Nonlinear Flutter of Orthotropic or Isotropic Panels with Arbitrary Flow Direction," Israel Journal of Technology, Vol. 6, No. 1-2, 1968, pp. 46-57.

78) Xue, D.Y. and Mei, C., "Finite Element Two-Dimensional Panel Flutter at High Supersonic Speeds and Elevated Temperature," Proc. 31st AIAA/ASME/ASCE/AHS/ASC Structures, Structural Dynamics and Materials Conference, 1990, pp. 1464-1475, AIAA Paper No. 90-0982.

79) Gray, E.G. and Mei, C., "Large-Amplitude Finite Element Flutter Analysis of Composite Panels in Hypersonic Flow," Proc. 33rd AIAA/ASME/ASCE/AHS/ASC Structures, Structural Dynamics and Materials Conference, Dallas, TX, April 16-17 1992, pp. 492-512, AIAA Paper No. 92-2130.

80) Abbas, J.F. and Ibrahim,R.A., "Nonlinear Flutter of Orthotropic Composite Panel Under Aerodynamic Heating," AIAA J., Vol. 31, No. 8, 1993, pp. 1478-1488.

81) Librescu, L., Marzocca, P., and Silva, W., "Supersonic/Hypersonic Flutter and Postflutter of Geometrically imperfect Cylindrical Panels," Journal of Spacecraft and Rockets, Vol. 39, No. 5, 2002, pp. 802-812.

82) Cheng, G. and Mei, C., "Finite Element Formulation for Hypersonic Panel Flutter Analysis with Thermal Effects," AIAA J., Vol. 42, No. 4, 2004, pp. 687-695.

83) Librescu, L., Marzocca, P., and Silva, W., "Linear/Nonlinear Supersonic Panel Flutter in a High-Temperature Field," Journal of Aircraft, Vol. 41, No. 4, 2004, pp. 918-924.

84) Pourtakdoust, S.H. and Fazelzadeh, S.A., "Nonlinear Aerothermoelastic Behavior of Skin Panel With Wall Shear Stress Effect," Journal of Thermal Stresses, Vol. 28, No. 2, 2005, pp. 147-169.

85) Azzouz, M.S. and Mei, C., "Nonlinear Flutter of Cylindrical Panels Under Yawed Supersonic Flow Using Finite Elements," Proc. 46th AIAA/ASME/ASCE/AHS/ASC Structures, Structural Dynamics and Materials Conference, Austin, TX, April 18-21 2005, AIAA Paper 2005-2373.

86) Rodgers, J.P., "Aerothermoelastic Analysis of a NASP-Like Vertical Fin," Proc. 33rd AIAA/ASME/ASCE/AHS Structures, Structural Dynamics and Materials Conference, Dallas, TX, April 1992, AIAA Paper 92-2400-CP.

87) Spain, C., Zeiler, T.A., Gibbons, M.D., Soistmann, D.L., Pozefsky, P., DeJesus, R.O., and Brannon,C.P., "Aeroelastic Character of a National Aerospace Plane Demonstrator Concept," Proc. 34th AIAA/ASME/ASCE/AHS/ ASC Structures, Structural Dynamics and Materials Conference, La Jolla, CA, April 19-22 1993, pp. 163-170.

88) Soistmann, D. and Spain, C., "An Experimental and Analytical Study of a Lifting Body Wind Tunnel Model Exhibiting Body-Freedom Flutter," Proc. 34th AIAA/ASME/ASCE/AHS/ ASC Structures, Structural Dynamics and Materials Conference, La Jolla, CA, April 19-22 1993, pp. 171-181, AIAA Paper No. 93-1316.

89) Scott, R.C. and Pototzky, A.S., "A Method of Predicting Quasi-Steady Aerodynamics for Flutter Analysis of High Speed Vehicles Using Steady CFD Calculations," Proc. 34th AIAA/ASME/ASCE/AHS/ASC Structures, Structural Dynamics and Materials Conference, La Jolla, CA, April 19-22 1993, pp. 595-603, AIAA Paper No. 931364 .

90) Raney, D., McMinn, J., Pototzky, A., and Wooley, C., "Impact of Aeroelasticity on Propulsion and Longitudinal Flight Dynamics of an Air-Breathing Hypersonic Vehicle," Proceedings of the 34th AIAA/ASME/ASCE/AHS/ASC Structures, Structural Dynamics and Materials Conference, La Jolla, CA, April 19-22 1993, pp. 628 - 637, AIAA Paper 93-1367.

91) Cole, S., Florance, J., Thomason, L., Spain, C., and Bullock, E., "Supersonic Aeroelastic Instability Results for a NASP-Like Wing Model," Proceedings of the 34th AIAA/ASME/ASCE/AHS/ASC Structures, Structural Dynamics and Materials Conference, La Jolla, CA, April 19-22 1993, pp. 638 - 647, AIAA Paper No. 93-1369.

92) Heeg, J. and Gilbert, M.G., "Active Control of Aerothermoelastic Effects for a Conceptual Hypersonic Aircraft," Journal of Aircraft, Vol. 30, 1993, pp. 453-458.

93) Blades, E., Ruth, M., and Fuhrman, D., "Aeroelastic Analysis of the X-34 Launch Vehicle," Proc. 40th AIAA/ASME/ASCE/AHS/ ASC Structures, Structural Dynamics and Materials Conference, St. Louis, MO, 1999, pp. 13211331, AIAA Paper 99-1352.

94) Pak, C.G. and Friedmann, P.P., "New Time Domain Technique for Flutter Boundary Identification," Proceedings of the AIAA Dynamics Specialist Conference, Dallas, TX, April 1992, pp. 201-214, AIAA Paper 92-2102.

95) Bae, J., Kim, J., Lee, I., Matsuzaki, Y., and Inman, D., "Extension of Flutter Prediction Parameter for Multimode Flutter Systems," Journal of Aircraft, Vol. 42, No. 1, 2005, pp. 285-288.

96) Matsuzaki, Y. and Torii, H., "Flutter Boundary Prediction of an Adaptive Smart Wing during Process of Adaptation Using Steady-State Response Data," Proc. 47th AIAA/ASME/ASCE/AHS/ ASC Structures, Structural Dynamics and Materials Conference, No. AIAA-2006-2132, Newport, RI, May 1 - 42006.

97) Bisplinghoff, R.L., "Some Structural and Aeroelastic Considerations of High-Speed Flight," Journal of the Aerospace Sciences, Vol. 23, No. 4, April 1956, pp. 289-329.

98) Rogers, M., "Aerothermoelasticity," AeroSpace Engineering, October 1958, pp. 34-43.

99) Garrick, I.E., "Aeroelasticity - Frontiers and Beyond," Journal of Aircraft, Vol. 13, September 1976, pp. 641-657.

100) Budiansky, B. and Mayers, J., "Influence of Aerodynamic Heating on the Effective Torsional Stiffness of Thin Wings,"

Journal of the Aeronautical Sciences, December 1956, pp. 1081-1093.

101) Anderson, J.D., Aerothermodynamics: A Tutorial Discussion, chap. 1, AIAA, 1992, pp. 3-57.

102) Thornton, E. and Dechaumphai, P., "Coupled Flow, Thermal, and Structural Analysis of Aerodynamically Heated Panels," Journal of Aircraft, Vol. 25, No. 11, November 1988, pp. 1052 - 1058. 
103) Doggett, R. V., Ricketts, R. H., and Noll, T. E. and Malone, J. B., "NASP Aeroservothermoelasticity Studies," NASA TM 104058, 1991.

104) Biot, M.A., "Influence of Thermal Stresses on the Aeroelastic Stability of Supersonic Wings," Journal of the Aeronautical Sciences, Vol. 24, 1957, pp. 418-420.

105) Runyan, H. L., and Jones, N. H.,, "Effect of Aerodynamic Heating on the Flutter of a Rectangular Wing at a Mach Number of 2," NACA RM L58C31, 1958.

106) Ericsson, L. E., Almroth, B. O., and Bailie, J. A., "Hypersonic Aerothermoelastic Characteristics of a Finned Missile," 16th Aerospace Sciences Meeting, Huntsville, Al, January 1978, AIAA Paper 78-231. 1981.

107) Sova, G. and Divan, P., "Aerodynamic Preliminary Analysis System II, Part II Users Manual," NASA CR 182077,

108) Dechaumphai, P., Wieting, A., and Pandey, A., "Fluid-Thermal-Structural Interaction of Aerodynamically Heated Leading Edges," Proceedings of the 30th AIAA/ASME/ASCE/AHS/ASC Structures, Structural Dynamics and Materials Conference, April 1989, pp. 621 - 631, AIAA Paper 89-1227.

109) Lohner, R., Yang, C., Cebral, J., and Baum, J. et al., "Fluid-Structure-Thermal Interaction using a Loose Coupling Algorithm and Adaptive Unstructured Grids," Proceedings of the 29th AIAA Fluid Dynamics Conference, Albuquerque, NM, June 1998, AIAA Paper 98-2419. CP.

110) Schmidt, D., "Dynamics and Control of Hypersonic Aeropropulsive/Aeroelastic Vehicles," AIAA Paper No. 92-5326-

111) Chavez, F. and Schmidt, D., "Uncertainty Modeling For Multivariable-Control Robustness Analysis of Elastic HighSpeed Vehicles," Journal of Guidance, Control, and Dynamics, Vol. 22, No. 1, 1999.

112) Lind, R., "Linear Parameter-varying Modeling and Control of Structural Dynamics with Aerothermoelastic Effects," Proc. 40th AIAA/ASME/ASCE/AHS/ASC Structures, Structural Dynamics, and Materials Conference and Exhibit, St. Louis, MO, 1999, AIAA Paper 99-1393.

113) Lind, R., Buffington, J., and Sparks, A., "Multi-loop Aeroservoelastic Control of a Hypersonic Vehicle," AIAA Guidance, Navigation, and Control Conference and Exhibit, Portland, OR, 1999, AIAA Paper 99-4123.

114) Rudd, L. and Pines, D., "Integrated Propulsion Effects on Dynamic Stability and Control of Hypersonic Waveriders," Proc. 36th AIAA/ASME/SAE/ASEE Joint Propulsion Conference and Exhibit, Huntsville, AL, July 1990, AIAA Paper 20003826.

115) Mirmirani, M., Wu, C., Clark, A., Choi, S., and Colgren, R., "Modeling for Control of a Generic Air-Breathing Hypersonic Vehicle," AIAA Guidance, Navigation, and Control Conference and Exhibit, San Fransico, CA, August 2005, AIAA Paper 2005-6256.

116) Bolender, M. and Doman, D., "A Non-Linear Model for the Longitudinal Dynamics of a Hypersonic Air-Breathing Vehicle," AIAA Guidance, Navigation, and Control Conference and Exhibit, San Fransico, CA, August 2005, AIAA Paper $2005-6255$.

117) Walton, J., "Program SGRULL," Available through COSMIC, University of Georgia, Athens, GA, (404) 542-3265, LEW-15093, May 1990.

118) Schmidt, D. and Lovell, T., "Mission Performance and Design Sensitivities of Air-Breathing Hypersonic Launch Systems," Journal of Spacecraft and Rockets, Vol. 34, No. 2, 1997.

119) Cockrell, C., Engelund, W., Bittner, R., Jentink, T., Dilley, A., and Frendi, A., "Integrated Aeropropulsive Computational Fluid Dynamics Methodology for the Hyper-X Flight Experiment," Journal of Spacecraft and Rockets, Vol. 38, No. 6, 2001.

120) Godfrey, A., "General Aerodynamic Simulation Program Version 3.0 User's Manual," Aerosoft Inc., Blacksburg, VA, May 1996.

121) Markotos, N., Spalding, D., and Tatchell, D., "Combustion of Hydrogen Injected into a Supersonic Airstream (The SHIP Computer Program)," NASA CR-2802, April 1977.

122) Ferlemann, P., "Improvements to the SHIP Computer Code and Predictions of Vorticity Enhanced Turbulent Supersonic Mixing," M.S. Thesis, School of Engineering and Applied Sciences, George Washington University, Washington D.C., 1993.

123) Bowcutt, K.G., "Multi-Disciplinary Optimization of Air-Breathing Hypersonic Vehicles," Journal of Propulsion and Power, Vol. 17, No. 6, 2001.

124) Stewart, M., Suresh, A., Liou, M., Owen, A., and Messit, D., "Multidisciplinary Analysis of a Hypersonic Engine," Proc. 11th International Conference on Space Planes and Hypersonic Systems and Technologies, Orleans, France, September 2002, AIAA Paper No. 2002-5127.

125) Buning, P., Jesperson, D., Pulliam, T., Klopfer, G., Chan, W., Slotnick, J., Krist, S., and Renze, K., "Overflow Users Manual," Version 1.8j, 1999, NASA Langley Research Center, Hampton VA.

126) White, J., "VULCAN Users Manual," Version 4.3, 2002, NASA Langley Research Center, Hampton VA.

127) "ANSYS Users Manual," Version 5.6, 1999, Swanson Analysis Systems, Inc., Cannonsburg, PA.

128) Clark, A., Wu, C., Mirmirani, M., Choi, S., and Kuipers, M., "Development of an Airframe-Propulsion Generic Hypersonic Vehicle Model," 44th AIAA Aerospace Sciences Meeting and Exhibit, Reno, NV, 2006, AIAA Paper $2006-218$. 